\title{
Quantum criticality beyond the Landau-Ginzburg-Wilson paradigm
}

\author{
T. Senthil, ${ }^{1}$ Leon Balents, ${ }^{2}$ Subir Sachdev ${ }^{3}$ Ashvin Vishwanath, ${ }^{1}$ and Matthew P. A. Fisher ${ }^{4}$ \\ ${ }^{1}$ Department of Physics, Massachusetts Institute of Technology, Cambridge MA 02139 \\ ${ }^{2}$ Department of Physics, University of California, Santa Barbara, CA 93106-4030 \\ ${ }^{3}$ Department of Physics, Yale University, P.O. Box 208120, New Haven, CT 06520-8120 \\ ${ }^{4}$ Kavli Institute for Theoretical Physics, University of California, Santa Barbara, CA 93106-4030
}

(Dated: May 28, 2018)

\begin{abstract}
We present the critical theory of a number of zero temperature phase transitions of quantum antiferromagnets and interacting boson systems in two dimensions. The most important example is the transition of the $S=1 / 2$ square lattice antiferromagnet between the Néel state (which breaks spin rotation invariance) and the paramagnetic valence bond solid (which preserves spin rotation invariance but breaks lattice symmetries). We show that these two states are separated by a second-order quantum phase transition. The critical theory is not expressed in terms of the order parameters characterizing either state (as would be the case in Landau-Ginzburg-Wilson theory), but involves fractionalized degrees of freedom and an emergent, topological, global conservation law. A closely related theory describes the superfluid-insulator transition of bosons at half-filling on a square lattice, in which the insulator has a bond density wave order. Similar considerations are shown to apply to transitions of antiferromagnets between the valence bond solid and the $Z_{2}$ spin liquid: the critical theory has deconfined excitations interacting with an emergent U(1) gauge force. We comment on the broader implications of our results for the study of quantum criticality in correlated electron systems.
\end{abstract}

\section{Contents}

I. Introduction and motivation
\begin{tabular}{|l|}
\hline II. Overview \\
\hline A. History and Precedents \\
B. Numerics \\
\hline C. Plan of Attack \\
D. Organization of paper \\
\hline
\end{tabular}

\section{Representations and Svmmetries}

IV. SJ Models

A. S.J model at $N=1$

B. SJ model at large $N$

V. Spin models with easv plane anisotropv

A. Semi-classical analysis

B. Easv plane in the $\mathrm{CP}^{1}$ representation

VI. Phase transitions

A. Easv plane limit

B. Isotropic magnets
A. Superfluid-insulator transition of correlated boson 25

1
6
6
8
8
9

9

12

12

13

15

15

16

17

17

19

\begin{tabular}{|c|c|}
\hline 1. Orbital magnetic field & 2 \\
\hline B. Higher spin & 2 \\
\hline C. Honevcomb lattice & \\
\hline D. Ising Anisotropy and Other Transitions & \\
\hline
\end{tabular}

X. Experiments

28

XI. Discussion

29

B. Duality transformation with easv plane anisotropy 30

C. Estimate of monopole scaling dimension 31

D. SJ models in one dimension 31

E. Direct derivation of dual meron action 32

VII. Physical properties near the 'deconfined' criticai Proing
A. Ordered state
21
B. Uniform Zeeman field
C. Staggered Zeeman field
22
23
D. Finite temperature transitions

References

34

VIII. Deconfined quantum criticality at the VBS to spint-liquid traksition 23 AND MOTIVATION

A. Spin liquids that break lattice svmmetrv

IX. Analogies and extensions
24 A central concept in the theory of phase transitions is that of the 'order parameter', which expresses the differ-

25 ent symmetries of the phases on either side of the critical 
point. If the transition is second order, there is interesting universal singular behavior that is manifested in many physical quantities. According to the prevalent paradigm largely due to Landau and Ginzburg ${ }^{1}$, these universal critical singularities are associated with long wavelength low energy fluctuations of the order parameter degree of freedom. When combined with general renormalization group ideas ${ }^{2}$, this notion provides the sophisticated Landau-Ginzburg-Wilson (LGW) theoretical framework for thinking about critical phenomena in various diverse contexts. Specifically, static critical properties at non-zero temperature are supposed to be determined from effective models in which all modes other than the order parameter have been eliminated. Similarly, for dynamical critical properties, the only degrees of freedom that purportedly need be retained are the order parameter and at most a few additional "hydrodynamic" modes having slow relaxation times due to conservation laws.

Recent years have seen much interest in the study of zero temperature phase transition phenomena in correlated many body systems. Unlike their thermal counterpart, such transitions are often driven by quantum fluctuation effects and are hence known as 'quantum phase transitions ${ }^{3}$. Indeed, it has been proposed that proximity to quantum critical points (QCPs) separating two distinct phases is responsible for the anomalous properties of some interesting correlated materials such as, for instance, the cuprate superconductors. Theoretically, the LGW paradigm has thus far provided the basic framework to examine quantum critical phenomena as well. In particular, the critical modes specific to a quantum critical point are presumed to be the long distance, long time fluctuations of the order parameter, described in a continuum field theory.

In the last few years some interesting and tantalizing evidence has emerged that points toward the failure of the LGW paradigm at certain quantum phase transitions. First, there are numerical calculations 4.5 that see a direct second order quantum phase transition between two phases with different broken symmetry characterized by two apparently independent order parameters. A LGW description of the competition between such two kinds of orders would then generically predict either a first-order transition, or an intermediate region of coexistence where both orders simultaneously exist, or an intermediate region with neither order. A direct second order transition between these two broken symmetry phases would seem to require fine-tuning to a 'multicritical' point. Are the numerics managing to achieve this 'fine-tuning' or is the LGW paradigm simply invalid?

Second, there have been a number of fascinating experiments probing the onset of magnetic long range order in a class of rare-earth inter-metallics known as the heavy fermion metals ${ }^{6.7}$. Remarkably, the behavior right at the quantum transition between the magnetic and nonmagnetic metallic phases is usually very different from that of a Fermi liquid. Furthermore, such behavior is in severe disagreement with expectations based on LGW analyses. Specifically, theories associating the critical singularities with fluctuations of the natural magnetic order parameter in a metallic environment seem to have a hard time explaining the observed non-Fermi liquid phenomena. Once again it appears that more than the obvious possibly happens at some quantum critical points.

In this paper we demonstrate and study various specific examples of quantum phase transitions which violate the LGW paradigm. We will show that in a number of different quantum transitions, the natural field theoretic description of the critical singularities is not in terms of the order parameter field(s) that describe the bulk phases but in terms of some new 'emergent' degrees of freedom that are specific to the critical point. These new degrees of freedom may be thought of 'fractional' quantum number particles that interact with each other through an emergent gauge force in a sense made precise below. Laughlin has previously argued for fractionalization at quantum critical points on phenomenological grounds ${ }^{8}$. A non-technical overview of our results has appeared previously $\underline{\underline{9}}$

We note, in passing, that there are already numerous well-documented examples of the breakdown of the LGW paradigm in quantum systems in one dimension 10 However, these rely rather crucially on the description of various states in terms of the harmonic phase degrees of freedom of the Tomonaga-Luttinger liquid, and do not have any direct generalization to higher dimensions.

For the most part in this paper, we will study phase transitions in two dimensional quantum magnetism. These may also be fruitfully viewed from a different point of view as representing transitions of interacting bosons on a lattice at commensurate density. Quantum magnets provide a particularly useful laboratory to develop and test ideas on the theory of quantum phase transitions. Consider a quantum system of spin $S=1 / 2$ moments $\vec{S}_{r}$ on a two dimensional square lattice $(r=(x, y))$ with the Hamiltonian

$$
H=J \sum_{\left\langle r r^{\prime}\right\rangle} \vec{S}_{r} \cdot \vec{S}_{r^{\prime}}+\ldots
$$

The ellipses represent other short ranged interactions that may be tuned to drive various zero temperature phase transitions. We assume $J>0$, i.e antiferromagnetic interactions. Later we will consider various generalizations to other lattices, higher spins, etc.

The nature of some of the various possible ground states of such a Hamiltonian are quite well understood. First, there are states that develop magnetic long range order and break the spin rotation symmetry. The simplest example (and the one that we will focus on) are collinear antiferromagnets where the order parameter is a single vector $\vec{N}_{r}$ (the Néel vector), defined to describe a state of staggered magnetization,

$$
\vec{S}_{r}=\epsilon_{r} \vec{N}_{r}
$$




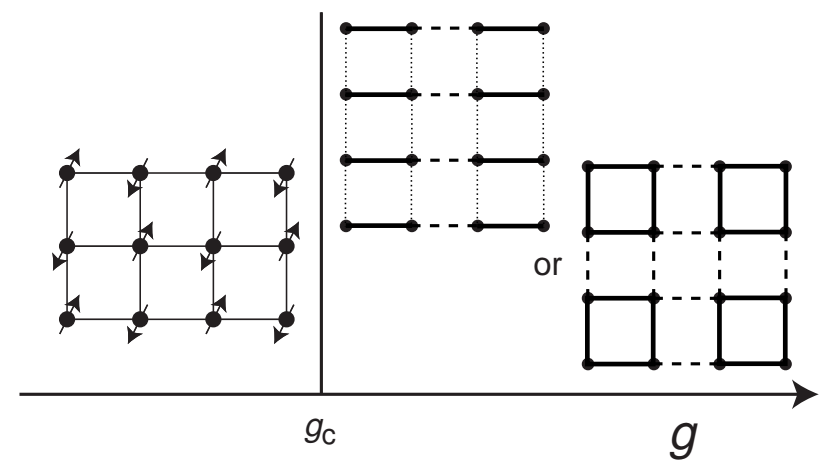

FIG. 1: Ground states of the square lattice $S=1 / 2$ antiferromagnet studied in this paper. The coupling $g$ controls the strength of quantum spin fluctuations about a magnetically ordered state, and appears in Eq. 2.1) (the classical limit is $g=0)$. There is broken spin rotation invariance in the Néel state for $g<g_{c}$, described by the order parameter $\vec{N}_{r}$ in Eq. (1.2). The VBS ground state appears for $g>g_{c}$, and is characterized by the order parameter $\psi_{\mathrm{VBS}}$ in Eq. (1.4 the distinct lines represent distinct values of $\left\langle\vec{S}_{r} \cdot \vec{S}_{r^{\prime}}\right\rangle$ on each link. The VBS state on the left has "columnar" bond order, while that on the right has "plaquette" order. The theory $\mathcal{L}_{z}$ in Eq. (1.7) applies only at the QCP $g=g_{c}$ at its critical point obtained at $s=s_{c}$.

where

$$
\epsilon_{r} \equiv(-1)^{x+y}
$$

is +1 on one checkerboard sublattice and -1 on the other. The Néel state has $\left\langle\vec{N}_{r}\right\rangle \neq 0$ and independent of $r$ (see Fig 1), but more generally $\vec{N}_{r}$ is presumed to vary "slowly" on the lattice scale over at least most of space. The low energy excitations of the Néel state are simply linear dispersing spin waves.

It is now recognized that a variety of quantum paramagnetic ground states are also possible where quantum fluctuations have prevented the spins from developing magnetic long range order, and so $\left\langle\vec{S}_{r}\right\rangle=0$. Such paramagnetic states can be broadly divided into two groups. First, there are states that can be described as 'Valence Bond Solid' (VBS) states $\frac{11}{1}$. In a simple caricature of such a state, each spin forms a singlet with one particular other spin resulting in an ordered pattern of "valence bonds'. For spin- $1 / 2$ systems on a square lattice, such states necessarily break lattice translational symmetry. The so-called "columnar" and "plaquette" ordering patterns (see Fig. (1) are described by a complex VBS order parameter $\psi_{\mathrm{VBS}}$, where

$$
\begin{aligned}
& \vec{S}_{r} \cdot \vec{S}_{r+\hat{x}} \sim \operatorname{Re}\left[\psi_{\mathrm{VBS}}\right](-1)^{x}, \\
& \vec{S}_{r} \cdot \vec{S}_{r+\hat{y}} \sim \operatorname{Im}\left[\psi_{\mathrm{VBS}}\right](-1)^{y},
\end{aligned}
$$

and $r=(x, y)$ (here columnar states have $\psi_{\mathrm{VBS}}^{4}$ real and positive, while plaquette states have $\psi_{\text {VBS }}^{4}$ real and negative). In these states there is an energy gap for spin-carrying $S=1$ quasi-particle excitations; these 'triplons ${ }^{12}$ are quite distinct from spin waves, and are instead adiabatically connected to spin excitons in band insulators. A second class of more exotic paramagnetic states are also possible $\frac{13.14 .15 .16 .17}{10}$ in principle: in these states the valence bond configurations resonate amongst each other and form a 'liquid'. The resulting state has been argued to possess excitations with fractional spin $1 / 2$ and interesting topological structure.

Our focus will be on the nature of the evolution of the ground state between these various phases. Our primary example is that between the ordered magnet and a valence bond solid. We also discuss the phase transitions between valence bond solid and 'spin' liquid phases (see Section VIII). Qualitatively similar phenomena will be ahown to obtain at both these transitions.

Both the magnetic Néel state, and the valence bond solid are states of broken symmetry. The former breaks spin rotation symmetry, and the latter the symmetry of lattice translations. The order parameters $\vec{N}$ and $\psi_{\text {VBS }}$ associated with these two different broken symmetries are very different. A LGW picture of the evolution between these two distinct ground states would be formulated in terms of an effective action that is a functional of $\vec{N}$ and $\psi_{\text {vBs }}$. Such a construction would suggest either a first order transition, or passage through an intermediate phase which breaks both kinds of symmetry. (Actually, the general LGW analysis also allows an intermediate 'disordered' state with neither order, but this possibility was excluded in early analyses ${ }^{11}$; this exclusion was already an indication that LGW theory did not apply here.) A direct second order transition would be expected only by further fine-tuning to special multicritical points. Our central thesis is that this expectation is wrong. A generic second order transition is possible between these two phases with different broken symmetries. The resulting critical theory is however unusual and not naturally described in terms of the order parameter fields of either phase. Instead, the natural description is in terms of spin- $1 / 2$ "spinon" or $\mathrm{CP}^{1}$ fields $z_{\alpha}(\alpha=1,2$ is a spinor index). The Néel order parameter is bilinear in the spinons:

$$
\vec{N} \sim z^{\dagger} \vec{\sigma} z .
$$

Here $\vec{\sigma}$ is the usual vector of Pauli matrices and multiplication of the spinor index is implied. The fields $z_{\alpha}$ create single spin-1/2 quanta, "half" that of the spin-1 quanta created by the Néel field $\vec{N}$.

The spinon fields $z_{\alpha}$ so defined have a U(1) "gauge" redundancy. Specifically the local phase rotation

$$
z \rightarrow e^{i \gamma(r, \tau)} z
$$

leaves the Néel vector invariant and hence is a gauge degree of freedom. Here $\tau$ is the imaginary time coordinate. Thus the spinons are coupled to a $\mathrm{U}(1)$ gauge field $a_{\mu}(r, \tau)$ (we will use the Greek indices $\mu, \nu, \ldots$ to represent the three spacetime indices $x, y, \tau)$. Our central thesis - substantiated by a variety of arguments to follow - 
is that the critical field theory for the Néel-VBS transition is just the simple continuum action $\mathcal{S}_{z}=\int d^{2} r d \tau \mathcal{L}_{z}$, and

$$
\begin{gathered}
\mathcal{L}_{z}=\sum_{a=1}^{N}\left|\left(\partial_{\mu}-i a_{\mu}\right) z_{a}\right|^{2}+s|z|^{2}+u\left(|z|^{2}\right)^{2} \\
+\kappa\left(\epsilon_{\mu \nu \kappa} \partial_{\nu} a_{\kappa}\right)^{2}
\end{gathered}
$$

where $N=2$ is the number of $z$ components (later we will consider the case of general $N),|z|^{2} \equiv \sum_{a=1}^{N}\left|z_{a}\right|^{2}$, and the value of $s$ is to be tuned to a critical value $s=s_{c}$ so that $\mathcal{L}_{z}$ is at its scale-invariant critical point. The same action with a simple modification also describes the critical field theory for systems with easy-plane anisotropy, with the addition of the simple term

$$
\mathcal{L}_{\mathrm{ep}}=w\left|z_{1}\right|^{2}\left|z_{2}\right|^{2},
$$

with $w<0$. We will discuss in more detail later why these would describe stable critical points - perhaps the most direct evidence comes from the numerical simulations reported in Ref. 21 of a lattice model of a $\mathrm{CP}^{1}$ field coupled to a noncompact gauge field (a lattice version of Eqn. [1.7), where a continuous transition was found in both the isotropic and easy plane cases.

How can this action describe the onset of VBS order when it does not contain $\psi_{\mathrm{VBS}}$, and the $z_{\alpha}$ are closely related to the Néel order parameter? In writing Eq. (1.7), we have tacitly assumed $a_{\mu}$ to be a single-valued continuous field. In a more careful lattice implementation of Eq. (1.5), however, the resulting gauge field that appears is compact, i.e. defined only modulo $2 \pi$. This allows for the presence of topological defects occurring at a single instant of space-time ("instantons") called monopoles, at which magnetic flux $\partial_{x} a_{y}-\partial_{y} a_{x}$ is created or destroyed in integer multiples of $2 \pi$. In general, Eq. (1.7) should thus be supplemented by terms which create or destroy such $2 \pi$ fluxes, or equivalently insert monopoles into the partition function:

$$
\mathcal{L}_{\mathrm{mp}}=\sum_{n=1}^{\infty} \lambda_{n}(r)\left(\left[v_{r \tau}\right]^{n}+\left[v_{r \tau}^{\dagger}\right]^{n}\right),
$$

where $v_{r \tau}^{\dagger}$ and $v_{r \tau}$ insert monopoles of strength $2 \pi$ and $-2 \pi$ at the space-time point $(r, \tau)$, respectively. Remarkably, it has been shown by Read and Sachdev 11.18 that this operator may be identified with the VBS order parameter, i.e.

$$
v_{r \tau} \sim \psi_{\mathrm{VBS}}(r, \tau) .
$$

A simple argument to this effect will be given in Sec. III Thus VBS physics is implicitly (albeit highly nonlinearly) contained in the gauge theory of Eqs. (1.7) and (1.9). Our claim that Eq. (1.7) without the monopole creation terms of Eq. (1.9) describes the critical properties of the Néel-VBS transition requires that the monopole "fugacities" $\lambda_{n}$ are irrelevant in the renormalization group sense at the QCP. Later sections of this paper will give a variety of compelling arguments, relying upon destructive quantum interference between different monopole events, for this irrelevance for spin- $1 / 2$ antiferromagnets. The arguments are based on quantum Berry phase effects described first by Haldane $\frac{19}{}$, which render $\lambda_{n}(r)$ oscillatory and negligible for $n \neq 0(\bmod 4)$ for spin $S=1 / 2$ (a different derivation appears in Appendix $\mathrm{A}$ and in the review in Ref. 20).

Although monopoles can be neglected at the QCP, this is not true at low energies in the VBS phase. Indeed, it is well-known from studies of pure compact $\mathrm{U}(1)$ gauge theories, that the fugacities $\lambda_{n}$ are always relevant in the absence of gapless "matter fields" (i.e. the $z_{\alpha}$ ), so that monopoles inevitably proliferate in this case. This proliferation leads to a "condensation" of the monopole operator, $\left\langle v_{r \tau}\right\rangle \sim\left\langle\psi_{\mathrm{VBS}}\right\rangle \neq 0$, hence VBS order ${ }^{11.18}$. At the same time it generates a gap for the gauge "photon". In renormalization group terminology, the monopole condensation in the VBS phase - despite the fact that the $\lambda_{n}$ are negligible at the QCP - indicates that (some) $\lambda_{n}$ are "dangerously irrelevant".

It is important to note that such monopoles have a natural topological interpretation in terms of the conformations of the Néel ordered state. In particular, low but non-zero energy configurations of the antiferromagnet are described by states with slowly-varying Néel vector (at least at spatial infinity) of constant amplitude,

$$
\vec{N}_{r}=|\vec{N}| \hat{n}_{r}
$$

Such classical configurations with finite energy admit topological defects known as skyrmions (see Fig 2). The total skyrmion number associated with a configuration defines an integer topological quantum number $Q$ :

$$
Q=\frac{1}{4 \pi} \int d^{2} r \hat{n} \cdot \partial_{x} \hat{n} \times \partial_{y} \hat{n} .
$$

Remarkably (see Sec. III and Ref. 22), the skyrmion density is simply related to the magnetic flux of the gauge field $a_{\mu}$,

$$
2 \pi Q=\int d^{2} x\left(\partial_{x} a_{y}-\partial_{y} a_{x}\right) .
$$

Thus the monopole instantons that change the gauge flux by $\pm 2 \pi$ describe events in which the skyrmion number changes by \pm 1 . Thus the flux creation operator $v_{r \tau}^{\dagger}$ can also be interpreted as a skyrmion creation operator. The skyrmion number changing events may be represented graphically as "hedgehog" configurations of the Néel vector in space-time (See Fig. 3). The irrelevance of the monopole fugacities at the Néel-VBS critical point is thus equivalent to the irrelevance of hedgehog fugacities in a semiclassical description. Further, the recognition that such monopole events can be characterized as changing (as a function of time) the skyrmion number $Q$ enables another interpretation of their irrelevance. In particular, in the critical fixed point theory in Eq. (1.7) - i.e. at 

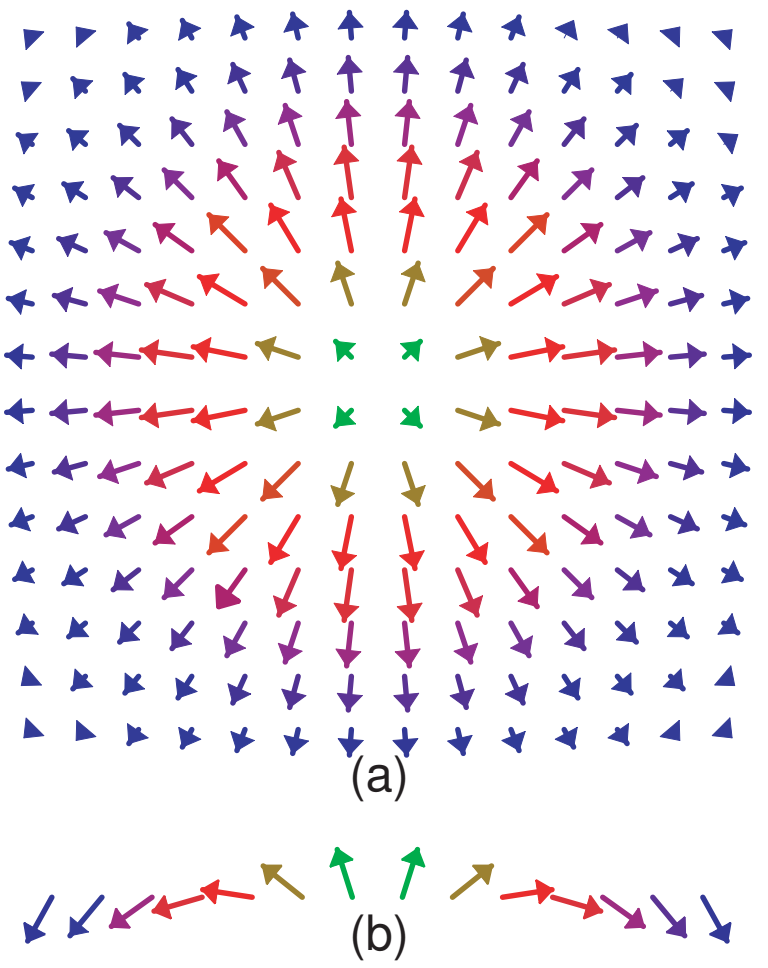

FIG. 2: A skyrmion configuration of the field $\hat{n}_{r}$. In (a) we show the vector $\left(n^{x}, n^{y}\right)$ at different points in the XY plane; note that $\hat{n} \propto(-1)^{x+y} \vec{S}_{r}$, and so the underlying spins have a rapid sublattice oscillation which is not shown. In (b) we show the vector $\left(n^{x}, n^{z}\right)$ along a section of (a) on the $x$ axis. Along any other section of (a), a picture similar to (b) pertains, as the former is invariant under rotations about the $z$ axis. The skyrmion above has $\hat{n}(r=0)=(0,0,1)$ and $\hat{n}(|r| \rightarrow \infty)=(0,0,-1)$.

low energies near the QCP - the skyrmion number $Q$ is strictly conserved. The emergence of this conserved topological quantum number is the most fundamental meaning of the irrelevance of the instantons.

We will also use this emergent topological conservation law as a definition of a "deconfined" QCP. Indeed, typically the gauge theories that arise in various slave particle descriptions of quantum magnets are compact. Specializing to a $\mathrm{U}(1)$ gauge theory, the compactness means that instanton or monopole events in which the magnetic flux changes by $2 \pi$ are allowed configurations of the gauge field in space-time. The proliferation of these instanton events leads to confinement of the slave particles in the gauge theory. In contrast, in a non-compact theory which emerges at low energies when monopoles are irrelevant - the total magnetic flux is strictly conserved. This is a topological conservation law and may be understood as a global U(1) symmetry in an appropriate dual description. Indeed, we will explicitly construct such a dual theory for the case of easy-plane anisotropy (and in some other related models). Quite generally, then, the emergence of a non-compact $\mathrm{U}(1)$ gauge theory at the critical point between the Néel and VBS phases signi-
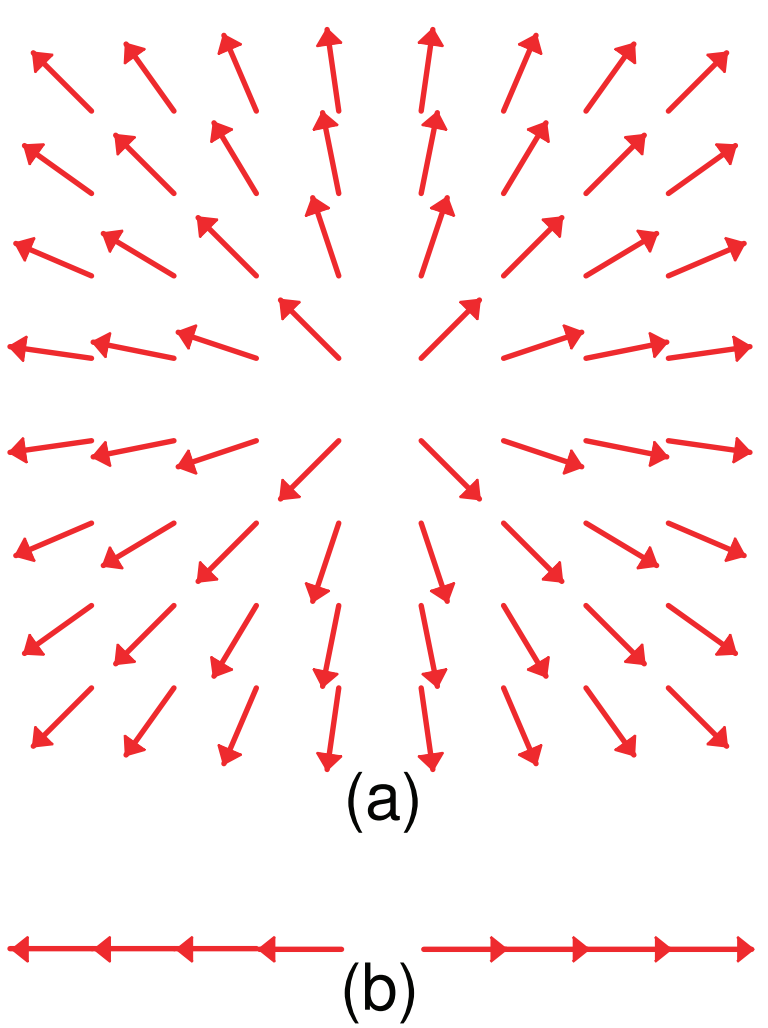

FIG. 3: A monopole event, taken to occur at the origin of spacetime. An equal-time slice of spacetime at the tunnelling time is represented following the conventions of Fig 2 So (a) contains the vector $\left(n^{x}, n^{y}\right)$; the spin configuration is radially symmetric, and consequently a similar picture is obtained along any other plane passing through the origin. Similarly, (b) is the representation of $\left(n^{x}, n^{z}\right)$ along the $x$ axis, and a similar picture is obtained along any line in spacetime passing through the origin. The monopole above has $\hat{n}_{r}=r /|r|$.

fies an extra emergent (dual) global U(1) symmetry for the critical theory that is not present in the microscopic Hamiltonian. This provides a rather precise characterization of a 'deconfined' critical point.

An important property of the deconfined fixed points discussed in this paper is the appearance of two distinct diverging length (or equivalently two time) scales close to the transition - one of which rises as a power of the other. This is directly due to the dangerous irrelevance of monopoles. For the Néel-VBS transition on approaching from the VBS side there is of course a diverging spin correlation length $\xi$. However just beyond this length scale the system has not yet chosen to pin itself into any particular VBS ordered state. Rather it may be characterized as fluctuating between different VBS configurations. It settles down to a particular ordered state at a larger length scale $\xi_{\mathrm{VBS}}$. This new length scale may also be characterized as the thickness of a domain wall in the VBS order. The universal crossovers associated with the critical fixed point describe the behavior on passing through the length scale $\xi$. These are described by the critical theory in Eq. (1.7). As explained above, this 
critical theory is monopole-free. The second crossover associated with the length scale $\xi_{\mathrm{VBS}}$ describes how the system evolves from the paramagnetic phase associated with the monopole-free theory Eq. (1.7) to the true VBS phase that obtains when monopoles eventually proliferate. Further details of the physics at the scales $\xi$ and $\xi_{\text {VBS }}$ appear in Section VII where we also show in Eq. (7.3. that $\xi_{\mathrm{VBS}}$ diverges as a power of $\xi$ which is greater than unity.

Over the last several years we have become familiar with the notion of fractionalization of quantum numbers in stable phases in condensed matter. In contrast, the fractionalization phenomena obtained in this paper are specific to the critical point separating two conventional phases. These 'fractional' particles - the spinons - are not present (i.e confined or condensed) at low energies on either side of the transition but appear naturally at the transition point. Likewise the emergent gauge field that mediates interactions between the fractional particles is also specific to the critical point. On approaching the critical point, the confinement length scale diverges. Thus 'deconfinement' appears right at the transition.

We will also briefly discuss the phase transitions between different quantum paramagnetic ground states. In particular we will argue that the existing theory for the transition between a VBS state and a fractionalized spin liquid implies that the corresponding critical point is also described by a deconfined U(1) gauge theory in precisely the same manner as above. Furthermore, Refs. 23, 24 argue that (at least under certain conditions) there are direct transitions between two different VBS phases that are also described by deconfined critical points with a $\mathrm{U}(1)$ gauge structure.

There are several general lessons to be learnt from the results in this paper. First, we see that two dimensional spin-1/2 quantum magnetism is full of examples of 'deconfined' quantum critical points which contradict the LGW paradigm for critical phenomena. This suggests that in more complex quantum systems (e.g. with fermions or disorder) novel critical phenomena may well be quite commonplace. Such deconfinement may be at the root of interesting non-Fermi liquid critical phenomena observed in the heavy fermion materials and possibly in the cuprates as well. Second, our results resolve some long-standing controversies in the field of two dimensional quantum magnetism and have direct implications for experiments and numerical work in the field.

Third, our results shed some light on questions of confinement in gauge theories in two spatial dimensions. It was shown by Polyakov several years ago 25 that in two spatial dimensions for pure gauge theories (i.e without any matter fields) instantons generically always proliferate and drive the theory into a confined phase. The behavior in the presence of dynamic matter fields (particularly with fermionic matter) is much less understood and is a subject of some controversy 26.27 .28 .29 .30 . The results in this paper show that with bosonic matter there are at least isolated critical points ${ }^{30}$ at which deconfine- ment is obtained (and the instantons disappear at long scales). While typically reaching criticality in a bosonic system requires some fine-tuning, fermionic systems can have stable critical phases. This supports the speculation that stable deconfined phases exist in two dimensional compact $\mathrm{U}(1)$ gauge theories coupled to fermionic matter ${ }^{31}$. If true this would have interesting implications for the theory of spin liquid phases of quantum spin systems. These points are discussed further in Section IVB and Appendix $\mathbf{F}$

Apart from these general notions, there are also a number of specific physical ramifications of the proposed critical theory for the Néel-VBS transition. One immediate consequence is that the anomalous dimension of the magnon operator is much larger than is usual at $D=2+1$ dimensional fixed points. Thus the magnon spectral function will be extremely broad right at the critical point. Many other implications are explored in some detail later in this paper and summarized in the overview in Section

\section{OVERVIEW}

In this section we provide an overview of the main ideas in this paper.

\section{A. History and Precedents}

We begin by recalling some important prior results in the theory of quantum magnetism on the two dimensional square lattice. In the Néel phase or close to it, the long distance low energy fluctuations (of the orientation) of the Néel order parameter are captured by the quantum $\mathrm{O}(3)$ Non-Linear Sigma Model $(\mathrm{NL} \sigma \mathrm{M})$ with the Euclidean action (we have promoted the lattice coordinate $r=(x, y)$ to a continuum spatial co-ordinate, and $\tau$ is imaginary time):

$$
\begin{aligned}
\mathcal{S}_{n} & =\mathcal{S}_{0}+\mathcal{S}_{B} \\
\mathcal{S}_{0} & =\frac{1}{2 g} \int d \tau \int d^{2} r\left[\left(\frac{\partial \hat{n}}{\partial \tau}\right)^{2}+c^{2}\left(\nabla_{r} \hat{n}\right)^{2}\right] \\
\mathcal{S}_{B} & =i S \sum_{r} \epsilon_{r} \mathcal{A}_{r}
\end{aligned}
$$

Here $\hat{n}_{r} \propto \epsilon_{r} \vec{S}_{r}$ is a unit three component vector that represents the Néel order parameter (the factor $\epsilon_{r}$ is defined in Eq. (1.3)). The term $\mathcal{S}_{B}$ contains crucial quantummechanical Berry phase effects, and is sensitive to the precise quantized value, $S$ of the microscopic spin on each lattice site: $\mathcal{A}_{r}$ is the area enclosed by the curve mapped out by the time evolution of $\hat{n}_{r}(\tau)$ on the unit sphere. These Berry phases play an unimportant role in the low energy properties of the Néel phase ${ }^{32}$, but are crucial in correctly describing the quantum paramagnetic phase $\stackrel{11,18}{ }$. We will expand these earlier results to show 
here that they also modify the quantum critical point between these phases, so that the critical exponents are distinct from the theory without $\mathcal{S}_{B}$ studied earlier ${ }^{32.33}$.

To understand the summation over $r$ in $\mathcal{S}_{B}$, recall that, as described in Section \ in two spatial dimensions, smooth configurations of the Néel vector admit skyrmion topological defects characterized by the integer topological charge $Q$. The Berry phase $\mathcal{S}_{B}$ vanishes ${ }^{3.34}$ for all smooth configurations even if they contain skyrmions. For such smooth configurations, the total skyrmion number $Q$ is conserved. Crucially, however, the skyrmion number changing monopole events are not everywhere smooth. It was shown by Haldane $\frac{19}{}$ that the summation over $r$ in $\mathcal{S}_{B}$ is non-vanishing in the presence of such monopole events. Precise calculation ${ }^{18.19}$ gives a Berry phase associated with each such skyrmion changing process which, for $2 S=1(\bmod 4)$, oscillates rapidly on four sublattices of the dual lattice (see Appendix A). This leads to destructive interference which effectively suppresses all monopole events unless they are quadrupled ${ }^{11.18 .19}$ (i.e they change skyrmion number by four).

The NL $\sigma \mathrm{M}$ field theory augmented by these Berry phase terms is, in principle, powerful enough to correctly describe the quantum paramagnet. Summing over the various monopole tunnelling events shows that in the paramagnetic phase the presence of the Berry phases leads to VBS order ${ }^{11.18}$. This crucial result from prior work identifies the VBS phase as resulting from a proliferation of monopoles in the presence of non-trivial Berry phases. The non-trivial identification of the VBS order parameter expressed from bond energies in Eq. (1.4) as the skyrmion creation operator is remarkable. When this operator acquires an expectation value VBS order results. In this manner $\mathcal{S}_{n}$ contains within it the ingredients describing both the ordered phases of $H$.

An important conclusion which follows from this body of work, via the above identification, is that a direct second order transition from the (collinear) Néel phase to a translation symmetric 'spin-liquid' is likely to be absent in two spatial dimensions. This still leaves several possibilities for the evolution of the ground state from the Néel to the VBS phases. For instance, there could be two transitions with an intermediate phase that breaks both Néel and lattice symmetries (an intermediate phase that breaks neither symmetry is excluded), or simply a first order transition.

The possibility of a direct second order transition between Néel and VBS phases is hinted at by several results in the existing literature. First, note that in the Néel phase monopole tunneling events are absent at long length and time scales. In the quantum paramagnet these monopole tunneling events have proliferated. The Haldane phases then lead to VBS order. The existence of a monopole condensate is clearly incompatible with long range Néel order. Thus to the extent that the broken lattice symmetry of the VBS state is a consequence of the proliferation of monopoles it competes with the Néel state. A direct transition from Néel to VBS then becomes conceivable 11.18.36.

A second hint comes from examining large- $N$ studies of $\mathrm{SU}(N)$ quantum spin models ${ }^{11,18}$. In the limit $N=\infty$ (and in a bosonic representation of the spins) there is a second order transition between the Néel phase and a quantum paramagnet. In this limit the paramagnet breaks no symmetries (including lattice symmetries). Furthermore, it supports gapped spin-1/2 excitations (known as spinons). However, both features are known to be artifacts of the limit $N=\infty$. Upon including finite $N$ corrections, broken lattice symmetry appears (leading to a VBS phase). The spinons also feel a gauge force that leads to their confinement and disappear from the spectrum. What about the vicinity of the transition? To answer this, it is instructive to examine the various length scales in the problem in the paramagnetic state. First, there is the spin correlation length that diverges on approaching the transition. Note that deep in the paramagnetic phase this length stays constant when $N \rightarrow \infty$. Then, there is the length scale at which VBS order appears. As there is no VBS order at $N=\infty$ this length scale must diverge as $N \rightarrow \infty$ - hence it must be much bigger than the spin correlation length in the large- $N$ limit. Finally, there is a third (somewhat loosely defined) length scale that may be thought of as the length scale associated with spinon confinement. Clearly this scale also diverges as $N \rightarrow \infty$ and is much bigger than the spin correlation length. Indeed calculations of the VBS and confinement length scales in the large- $N$ limit show that they are $\mathcal{O}\left(\xi^{c N}\right)$, where $\xi$ is the spin correlation length ${ }^{35}$ and $c$ is a constant.

This suggests the possibility of a direct transition between Néel and VBS states in the large- $N$ limit where the monopoles (and hence their Berry phases) are irrelevant at the critical fixed point, but are important in producing VBS order and confinement in the paramagnetic state. In critical phenomena parlance, the monopoles are dangerously irrelevant at the critical fixed point.

A picture similar to this was in fact proposed several years ago by Chubukov et al. ${ }^{33}$. However, it was not appreciated that the quadrupling of the monopoles, induced by the Berry phases, renders both the monopoles and their Berry phases irrelevant at the critical point (the distinction between the relevance of single versus quadrupled monopoles is absent in the large $N$ limit $^{37}$ ). In particular, it was assumed that the confinement length scale will stay finite at the transition, which was then modelled (for physical $S U(2)$ spins) by the LGW theory obtained simply by neglecting $\mathcal{S}_{B}$ in Eq. (2.1): this is the $\mathrm{O}(3)$-invariant Wilson-Fisher fixed point ${ }^{2}$. In light of the discussion above, it is clear that as the confinement goes hand in hand with the VBS order both confinement and VBS length scales diverge at the transition. Thus we might expect 'deconfinement' to appear at the transition.

A weakness in the arguments of Chubukov et al ${ }^{33}$ was pointed out by Sachdev and Park ${ }^{38}$. The latter authors argued that there was a finite density of monopoles in 
space-time right at the critical point of the O(3) LGW model, and the Berry phases then implied the presence of finite VBS order at any such critical point. Based on this they suggested that a possible evolution between the Néel and VBS phases was through a region of coexistence of both broken symmetries. However, they left open the possibility of a direct second order transition between the Néel and VBS phases, but argued that any such transition could not be described by the O(3) LGW model.

Our discussion here makes it clear it is necessary that the corresponding fixed point have no monopoles at long scales. The natural candidate is then precisely the fixed point governing the transition in the model with monopoles forbidden. The arguments of Motrunich and Vishwanath $^{21}$, and our present analysis, on such models show that the appropriate critical theory is that of $\mathcal{L}_{z}$ in Eq. (1.7). It must be kept in mind that this critical theory is entirely distinct, with all critical exponents different, from the $\mathrm{O}(3) \mathrm{LGW}$ model obtained by dropping $\mathcal{S}_{B}$ from Eq. 2.1). The first indication that such a distinct continuous transition could exist in the monopole suppressed $\mathrm{O}(3) \mathrm{NL} \sigma \mathrm{M}$ was from the work of Kamal and Murthy ${ }^{39}$. Recently, the transition in this model with monopole suppression was studied in Ref. 21, where a new approach that sidestepped the potential problems of Ref. 39 was used. A continuous, non-Heisenberg transitions with properties consistent with those of Ref. 39 was found. Moreover, an independent numerical simulation of a $\mathrm{CP}^{1}$ model with a noncompact gauge field was performed (essentially Eq. 1.7) which also yielded a continuous transition and exponents consistent with the simulations of the monopole suppressed $\mathrm{O}(3) \mathrm{NL} \sigma \mathrm{M}$. This provided a nontrivial check of both the essential correctness of the numerical calculations and direct support for the identification of Eq. (1.7) as the critical theory for the monopole suppressed $\mathrm{O}(3) \mathrm{NL} \sigma \mathrm{M}$ transition. The easy plane deformation of these models was also studied in Ref. 21, where again a continuous transition was obtained. This transition was argued to possess the remarkable property of being self-dual.

The possibility of deconfinement of spinons at the critical point between Néel and VBS phases is also hinted at by a different consideration that is again motivated by the large- $N$ calculations. The excitations of both the Néel and VBS phases are conventional (i.e do not contain any fractionalized spinons). In a Schwinger boson description in terms of spin-1/2 spinons this is achieved through confinement. However the detailed mechanism of such spinon confinement is different in the two phases. In the Néel phase (described as a spinon condensate) confinement is achieved through the usual Higgs mechanism. On the other hand, in the VBS phase confinement is achieved through proliferation of instantons. This difference in the confinement physics then makes it conceivable that neither mechanism is actually operational at the critical point and deconfinement obtains.

\section{B. Numerics}

There have been a large number of numerical studies of the destruction of Néel order in the $S=1 / 2$ square lattice antiferromagnet ${ }^{40}$. While there is evidence for the existence of VBS order in the paramagnetic phase, $41,42,43,44,45$ the nature of the transition between the Néel and paramagnetic states has been difficult to address. A major obstacle is the well-known 'sign' problem, which prevents large-scale Monte Carlo simulations. Until recently, all large scale studies of the destruction of Néel order have been on models with an even number of $S=1 / 2$ spins per unit cell, with a paramagnetic phase which does not break any lattice symmetries 46.47

The first large-scale study of the destruction of Néel order in a $S=1 / 2$ square lattice antiferromagnet, in a Hamiltonian which preserves a single $S=1 / 2$ spin per unit cell and the full symmetry of the square lattice, was that of Sandvik et $a l^{5}$. This was on a model with a strong easy-plane anisotropy. Such easy plane models have been studied analytically previously ${ }^{38.48}$ and will be pursued further in the present paper. Ref. 5 found convincing evidence for VBS order in the paramagnetic phase. Furthermore, the VBS and Néel order appear to vanish at points close to each other, suggesting a direct second order transition in the class discussed in the present paper.

We also note the wavefunction Monte Carlo work of Capriotti et al. $\frac{49}{}$ on the $\mathrm{SU}(2) S=1 / 2$ antiferromagnet on the square lattice with first and second neighbor exchange. They found a 'resonating valence bond' wavefunction characteristic of a spin liquid state. Our results here suggest that they were perhaps observing the deconfined state characteristic of the critical point, and that they had not yet reached the crossover to VBS order at the longest scales.

\section{Plan of Attack}

In this paper, the proposal of a deconfined continuous Néel-VBS transition (as well as a VBS-spin liquid transition) is substantiated by a variety of arguments. First, in Section [II] we consider a concrete lattice $C P^{N-1}$ model which, for $N=2$, embodies the physics of the Néel state, the monopoles and their Haldane Berry's phases (focusing on $S=1 / 2$ ), and the VBS state. This model, introduced by Sachdev and Jalabert ${ }^{36}$, and referred to here as the SJ model, provides a convenient starting point for theoretical analysis of the $S U(2)$ invariant critical region. We address the nature of the physically interesting $N=2$ case by showing that, in the two limits $N=1$ (Section IVA and $N=\infty$ (Section IVB ), this model indeed sustains a deconfined critical point in the precise sense defined above. For $N=1$, this can be directly shown using lattice duality transformations, which demonstrate an exact equivalence of the SJ model to a $D=3$ classical XY model with a fourfold symmetry breaking term which corresponds physically to strength four monopoles. 
Such four-fold anisotropy is known to be irrelevant at the $D=3 \mathrm{XY}$ transition ${ }^{50}$, establishing the deconfinement of this case. For $N=\infty$, the scaling dimension of the 4-skyrmion creation operator was computed previously by Murthy and Sachdev ${ }^{35}$, and is such that monopoles are again irrelevant. Hence we expect by continuity that monopoles are irrelevant for all $N$, including the interesting case $N=2$.

Second, in Section $\square$ we consider specifically $N=2$, in the presence of additional (strong) easy-plane anisotropy. In this case, the SJ model may be rewritten as a pair of $\mathrm{O}(2)$ rotors (the phases of $z_{\alpha}$ ) interacting with a compact $\mathrm{U}(1)$ gauge field. The latter may be analyzed directly using duality techniques (Appendix B). We obtain in this way an explicit dual representation in terms of complex "vortex" annihilation operators $\psi_{\alpha}(\alpha=1,2)$ and a dual non-compact gauge field $A_{\mu}$, whose flux represents the (exactly) conserved uniform spin density $S^{z}$. One may understand the relation to the $\mathrm{CP}^{1}$ variables by recognizing that $\psi_{1}^{\dagger}$ creates a $+2 \pi$ vortex in $z_{2}$, while $\psi_{2}^{\dagger}$ creates a $-2 \pi$ vortex in $z_{1}$, both of which create physical $2 \pi$ vorticity in $n^{-}=n^{x}-i n^{y}=z_{1}^{*} z_{2}$. The dual theory, $\mathcal{L}_{\text {dual }}$ for $\psi_{\alpha}$ and $A_{\mu}$ is presented in Eq. (5.6).

The dual representation $\mathcal{L}_{\text {dual }}$ has an appealing semiclassical interpretation, described in detail in Sec. VA Briefly, the two types of vortices correspond to "merons" (half-skyrmions), in which the Néel vector points either up or down inside the vortex core. The skyrmion number changing monopole events thereby correspond precisely to an event in which a vortex core tunnels from the up to down staggered magnetization or vice-versa.

The advantage of this representation is that the (quadrupled) monopole fugacity appears explicitly as a local operator in terms of the vortex fields. Remarkably, if this fugacity, $\lambda$, is set to zero (as appropriate at the QCP provided it is, as we argue, irrelevant), the dual action in Eq. (5.6) has precisely the same form as the original one, Eq. (1.7). More precisely, an exact equivalence can be demonstrated between lattice regularizations of the original and dual theory in the absence of monopoles 21 . Thus, as found in Ref. 21, the proposed critical theory in the easy-plane case has an unusual selfduality property.

The irrelevance of monopoles can then be argued in several ways. First, using the self-duality, each power of the skyrmion creation operator has the same correlations at the QCP as the corresponding power of the $\mathrm{XY}$ staggered raising operator $n^{+}$. At the deconfined critical point, fluctuations of $n^{+}$are expected to be stronger than they are at a conventional (confined) XY critical point. The corresponding quadrupled operator is already irrelevant in the latter case (as mentioned above), so we expect the four-skyrmion fugacity to be only more irrelevant around the deconfined critical theory. This expectation is supported by an explicit calculation in a large- $N$ (different from the $N$ in the SJ model) generalization of the dual critical theory in Section VIA Further arguments are given in Appendix C
In Section IXA we demonstrate for the easy plane case a direct derivation of the dual critical theory from a microscopic bosonic representation of the underlying XY model, without utilizing either the $\mathrm{NL} \sigma \mathrm{M}$ or SJ models.

In Section VIII we show that analogous deconfinement obtains for a VBS to spin-liquid transition. The latter has already been discussed by several authors 17.51 , and shown to be equivalent to the transition in a fully frustrated quantum Ising model, which has a simple XY critical fixed point unaffected at low energies by an irrelevant 8-fold symmetry breaking term. We show that this description is in fact dual to a deconfined gauge theory in the same sense as above, and that the (dangerously) irrelevant 8 -fold symmetry breaking term can likewise be interpreted as an irrelevant monopole fugacity.

\section{Organization of paper}

We will begin in Section the important symmetries of the Hamiltonian, and their action on a variety of order parameters and operators. This section will also introduce the SJ model. The solution of the SJ model in a variety of tractable limits appears in Sections IV and VB Section $\nabla$ also contains a general, semiclassical description of the physics in the easy plane limit. The nature of the second-order critical point between the Néel and VBS states is discussed in Section VI A variety of predictions for the critical properties on the Néel-VBS transitions with $S U(2)$ and XY symmetries follow from our analysis. These are elaborated in Sec. VII readers not interested in the detailed theoretical analysis may skip ahead to this section without significant loss of continuity. Section [VIII] describes the deconfined critical point between the VBS and spinliquid phases, as noted above. Finally, Section IX] contains a variety of extensions of the results in this paper. Section IXA shows that the easy-plane Néel to VBS transition can be reinterpreted as a superfluid-insulator transition in an interacting boson system; the insulator in this case contains a density wave in the amplitude of the bosons to reside on 'bond' states. This approach also provides an alternative derivation of the dual model Eq. (5.6). Section IXB briefly discusses the extension of our results to antiferromagnets with $S>1 / 2$, while section IXC considers the case of the honeycomb lattice. Section IXD discusses possible extension of our results to systems with Ising anisotropy.

\section{REPRESENTATIONS AND SYMMETRIES}

In this section, we describe the representation of magnets with local tendencies to Néel order in the $\mathrm{NL} \sigma \mathrm{M}$ and $\mathrm{CP}^{1}$ (SJ model) representations. We describe the action of the physical symmetries on the corresponding $\hat{n}$ and $z_{\alpha}$ fields in each case 
We start from the action $\mathcal{S}_{n}$ in Eq. (2.1). The allimportant Berry phase term in $\mathcal{S}_{B}$ is defined on the underlying square lattice, and it is clear that lattice scale cancellations are important for the physics we are interested in. It is therefore useful to return to a lattice formulation to obtain

$$
\begin{aligned}
\mathcal{S}_{n} & =\mathcal{S}_{0}+\mathcal{S}_{B} \\
\mathcal{S}_{0} & =\int d \tau\left(\sum_{r} \frac{1}{2 g}\left(\frac{d \hat{n}_{r}}{d \tau}\right)^{2}-J \sum_{<r r^{\prime}>} \hat{n}_{r} \cdot \hat{n}_{r^{\prime}}\right) \\
\mathcal{S}_{B} & =i S \sum_{r} \epsilon_{r} \int d \tau \vec{A}[\hat{n}] \cdot \frac{d \hat{n}_{r}}{d \tau} .
\end{aligned}
$$

We have now rewritten the areas $\mathcal{A}_{r}$ in terms of $\vec{A}$, which represents the vector potential of a magnetic monopole with flux $4 \pi$ placed at the center of $\hat{n}$ space at each lattice site. This lattice model is a faithful representation of the original quantum antiferromagnet so long as $g$ is large. The continuum limit of $\mathcal{S}_{0}$ in this model is clearly just what appears in Eqn. 2.1 The representation of the Berry phase used here leads directly to Eq. (A1) in Appendix A

The Berry phases are crucial for a correct description of quantum paramagnetic phases. As described in the previous sections, it was shown by Haldane 19 that the Berry phases are non-vanishing only in the presence of monopole events. The calculations in Refs. 1819 give the total phase (for spin-1/2 magnets that we consider for a derivation see here, Appendix A.

$$
\prod_{n} \exp \left(i \frac{\pi}{2} \zeta_{n} \Delta Q_{n}\right) \text {. }
$$

Here the monopole is associated with a plaquette of the original lattice (or equivalently with a site of the dual square lattice), which is labelled by the index $n$. The product is over all locations of monopoles, and $\Delta Q_{n}=$ \pm 1 is the change in skyrmion number associated with the monopole. Note that the periodic boundary condition along the time direction requires that the net change in skyrmion number is zero so that $\sum_{n} \Delta Q_{n}=0$. The fixed integer field $\zeta_{n}$ is $0,1,2,3$ depending on whether the dual lattice coordinate is (even,even), (even,odd), (odd,even) or (odd,odd), so that the phase factor associated with each monopole is $1, i,-1,-i$ on these sublattices (see Fig 4 in Section IVA].

The oscillating nature of the Berry phase factors on adjacent plaquette leads to destructive interference between different tunnelling paths for single monopoles. Indeed this interference effectively kills all monopole events unless they are quadrupled (i.e change skyrmion number by four). Hence only such quadrupled monopole events need be including in the quantum statistical mechanical partition sum.

We have already indicated the remarkable identification of the VBS order parameter defined in Eq. (1.4) with the skyrmion annihilation operator, $\psi_{\mathrm{VBS}} \sim v$, as shown in Ref. 11. This provide the crucial confluence of the loss of antiferromagnetic order (and consequent proliferation of monopole events) with the onset of VBS order, counter to conventional LGW wisdom. Because of the importance of this result, we give a simplified derivation of this relation here.

It is important to recognize that the VBS order parameter in Eq. (1.4) is entirely defined by its transformation under the symmetries of the Hamiltonian. Any other field with the same symmetry properties as $\psi_{\text {VBS }}$ will, on general scaling and renormalization group grounds, be proportional to $\psi_{\mathrm{VBS}}$ in the critical region. Thus to prove the identification of $\psi_{\mathrm{VBS}}$ with the skyrmion creation operator $v$, it is sufficient to show that the latter transforms identically to $\psi_{\text {VBS }}$ under all symmetry operations.

As a topological index, the skyrmion number is unchanged under smooth global $S U(2)$ spin rotations, hence the skyrmion number changing operator is also an $S U(2)$ scalar. Likewise, $\psi_{\text {VBS }}$, being defined through scalar bond operators, is $S U(2)$ invariant. Let us consider the effect of lattice symmetry transformations on $v$. In the functional integral this operator is defined by insertion of a space-time monopole. It is easy to see that under $\pi / 2$ rotations in the counter-clockwise direction about a direct lattice site (which we denote $R_{\pi / 2}$ ), the Berry phase associated with the skyrmion creation event changes by $e^{i \pi S}$. Thus if we denote by $v^{\dagger}$ the skyrmion creation operator and specialize to $S=1 / 2$, we have

$$
R_{\pi / 2}: v^{\dagger} \rightarrow i v^{\dagger}
$$

The skyrmion creation operator is actually defined on a plaquette - for the time being, we will label the plaquette by the lattice site at the top right corner.

Lattice translation operations $T_{x, y}$ corresponding to translations by one unit along $x, y$ directions of the microscopic spin model have somewhat more subtle effects. First, in the rotor representation of $\hat{n}_{r}$, these translations are represented as

$$
\begin{aligned}
& T_{x}: \hat{n}_{r} \rightarrow-\hat{n}_{r+\hat{x}} \\
& T_{y}: \hat{n}_{r} \rightarrow-\hat{n}_{r+\hat{y}}
\end{aligned}
$$

The change in sign of $\hat{n}$ is due to the staggering implicit in its definition. Now note that the skyrmion number $Q$ is odd under $\hat{n} \rightarrow-\hat{n}$. Consequently $T_{x, y}$ convert a skyrmion creation operator to an antiskyrmion creation operator at the translated plaquette. Furthermore due to the difference in the Berry phase factors for monopoles centered on adjacent plaquettes, there is a phase factor that is introduced by the translation. Simple calculation gives the following transformation properties for $v^{\dagger}$, specializing again to $S=1 / 2$ :

$$
\begin{aligned}
& T_{x}: v_{r}^{\dagger} \rightarrow-i v_{r+\hat{x}} \\
& T_{y}: v_{r}^{\dagger} \rightarrow+i v_{r+\hat{y}} .
\end{aligned}
$$

It is now clear that a paramagnetic state with a uniform expectation value of $v^{\dagger}$ breaks these lattice symmetries. For instance, if $\left\langle v^{\dagger}\right\rangle=\langle v\rangle \neq 0$, then $R_{\pi / 2}, T_{x}, T_{y}$ 
are all broken. This suggests a plaquette ordered state such as that shown in the lower right of Fig. 1] A straightforward comparison shows that, up to an innocuous constant pre-factor, the lattice transformation properties of $v$ are identical to those of $\psi_{\mathrm{VBS}}$ determined from a more mundane analysis of Eq. (1.4). In particular,

$$
v \sim e^{-i \pi / 4} \psi_{\mathrm{VBS}}
$$

properly reproduces all the transformation properties of the VBS order parameter. Thus we may indeed identify the skyrmion creation operator with the order parameter for the VBS order.

We have already introduced in the introduction the $\mathrm{CP}^{1}$ "spinon" fields to represent the Néel order parameter. These may be introduced on the lattice,

$$
\hat{n}_{r}=z_{r}^{\dagger} \vec{\sigma} z_{r}
$$

To maintain the unit magnitude of $\hat{n}_{r}$, the constraint $\left|z_{1}\right|^{2}+\left|z_{2}\right|^{2}=1$ should be imposed upon the spinor $z=$ $z(r, \tau)=\left(z_{1}, z_{2}\right)$.

One can show 22 that the partition function of the continuum NL $\sigma \mathrm{M}$ (with the action in Eq. 2.1 neglecting Berry's phase terms) is exactly reproduced by the continuum $\mathrm{CP}^{1}$ model with the action

$$
\mathcal{S}_{c p}=\int d \tau d^{2} r\left|\left(\partial_{\mu}-i a_{\mu}\right) z\right|^{2} .
$$

Here $a_{\mu}$ enters mathematically as a HubbardStratonovich field, and by considering its quadratic Euler-Lagrange equation, one can deduce the relation of the skyrmion number $Q$ to the gauge flux of $a_{\mu}$ given in Eq. (1.13).

As discussed above, to incorporate VBS phases it is important to correctly account for the Haldane Berry phases associated with these instantons. An appropriate model has been constructed by Sachdev and Jalabert ${ }^{36}$. The Euclidean action of the Sachdev-Jalabert (SJ) model is

$$
\begin{aligned}
\mathcal{S}_{\mathrm{SJ}} & =\mathcal{S}_{z}+\mathcal{S}_{a}+\mathcal{S}_{B} \\
\mathcal{S}_{z} & =-t \sum_{i} z_{i \alpha}^{*} e^{i a_{\mu}} z_{i+\hat{\mu}, \alpha}+\text { c.c. }, \\
\mathcal{S}_{a} & =\frac{K}{2} \sum_{\mathcal{H}}\left(\epsilon_{\mu \nu \lambda} \Delta_{\nu} a_{\lambda}-2 \pi q_{\mu}\right)^{2} \\
\mathcal{S}_{B} & =i \frac{\pi}{2} \sum_{n} \zeta_{n} \Delta_{\mu} q_{\mu} .
\end{aligned}
$$

Here we have put the complex spinon fields $z_{i \alpha}$ on the sites, $i$, of a cubic space-time lattice in dimensions $D=$ $2+1$ (now, $n$ denotes the sites of the dual cubic lattice), and they satisfy a unit length constraints $\sum_{\alpha}\left|z_{i \alpha}\right|^{2}=1$ on each lattice site. The $a_{\mu}$ represent the compact $\mathrm{U}(1)$ gauge field, and are defined on the links of the spacetime lattice. Note that the $z_{i \alpha}$ are minimally coupled to the gauge field. The term $S_{a}$ represents the gauge field kinetic energy. The quantity $q_{\mu}$ is an integer gauge flux that is defined on the links of the dual cubic lattice. Its divergence which enters the term $\mathcal{S}_{B}$ represents the number of monopoles on the sites of the dual lattice. Consequently, Eq. (3.13) is identical to the contribution in Eq. (3.2), and $\mathcal{S}_{B}$ provides the Haldane Berry phase factors that make the action appropriate for describing spin-1/2 antiferromagnets on the square lattice. The Néel ordered phase is a 'Higgs' phase where the $z_{i}$ have condensed, while the VBS phase is a 'confined' phase where the Berry phases have led to broken lattice symmetry.

The action $\mathcal{S}_{\mathrm{SJ}}$ is clearly closely related to the lattice action $\mathcal{S}_{n}$ in Eq. (3.1), after replacing $\hat{n}$ by $z$ via Eq. (1.5). However, the corresponding $\mathcal{S}_{B}$ terms in Eqs. (3.1) and (3.13) do appear rather different - they are related by the Berry phase summation carried out by Haldane ${ }^{19}$. Here, we establish the connection between these two forms of Berry phases in Appendix A further details on the derivation of Eq. 3.13 from the microscopic antiferromagnet appear in Refs. 203638.

If, as we will argue in the following, monopole events can indeed be neglected at low energies near the QCP, we can set $q_{\mu}=0$. Taking then a naive continuum limit of Eq. 3.11 3.13 gives precisely the proposed field theory of Eq. (1.7). We will, however, work directly with the lattice SJ model including monopoles in several of the sections to follow.

As in any critical phenomenon, symmetry plays a key role in the discussion of the Néel-VBS transition. We therefore list here the various physical (i.e. non-gauge) symmetries of the problem and their action upon the Néel and spinon fields. The only continuous physical symmetry is spin-rotational invariance, either $S U(2)$ or $\mathrm{U}(1)$ in the case of easy-plane anisotropy. Under such rotations, the Néel vector $\hat{n}$ and spinon field $z_{\alpha}$ transform as global vectors and spinors, respectively. The remaining unitary symmetries are discrete operations of the space group of the square lattice, and can be composed from $\pi / 2$ rotations, translations, reflections, and inversions. As above, we denote $\pi / 2$ clockwise rotations (around a direct lattice site) by $R_{\pi / 2}$ and unit translations in $x$ and $y$ by $T_{x}, T_{y}$ respectively. Reflections $x \rightarrow-x$ or $y \rightarrow-y$ around a lattice plane (i.e. leaving a row or column invariant) are denoted $\mathcal{R}_{x}, \mathcal{R}_{y}$, and inversions about a site by $I$. Finally, there is a non-unitary time-reversal operation $\mathcal{T}$, which as usual takes microscopic spins $\vec{S}_{r} \rightarrow-\vec{S}_{r}$. The transformation properties of the Néel, spinor, and gauge fields are given in Table 1 .

Using these symmetry properties, one can determine the operators of the field theory corresponding to physically interesting microscopic quantities in a spin model. Some of these are tabulated in the second column of Table II of Section VB and describe general situations which allow for easy plane anisotropy on the underlying magnet. (The $S U(2)$ symmetric situation may be obtained as a special case). These include the easy-plane and hard-axis components of the Néel order parameter, $N^{ \pm}=N^{x} \pm i N^{y}, N^{z}$ and the VBS order parameter. Also included are the easy-plane and hard-axis components of the uniform magnetization $\vec{M}$, all three being conserved for $S U(2)$ symmetry but only the latter be- 


\begin{tabular}{l|c|c|c|c}
\hline \hline Operation & Coordinates & Néel & spinor & gauge \\
\hline$R_{\pi / 2}$ & $x_{i} \rightarrow \epsilon_{i j} x_{j}$ & invariant & invariant & $a_{i} \rightarrow \epsilon_{i j} a_{j}$ \\
$T_{x_{i}}$ & $x_{i} \rightarrow x_{i}+1$ & $\hat{n} \rightarrow-\hat{n}$ & $z_{\alpha} \rightarrow i \sigma_{\alpha \beta}^{y} z_{\beta}^{\dagger}$ & $a_{\mu} \rightarrow-a_{\mu}$ \\
$\mathcal{R}_{x_{i}}$ & $x_{i} \rightarrow-x_{i}$ & invariant & invariant & $a_{i} \rightarrow-a_{i}$ \\
$I$ & $r \rightarrow-r$ & invariant & invariant & $a_{x / y} \rightarrow-a_{x / y}$ \\
$\mathcal{T}$ & $t \rightarrow-t$ & $\hat{n} \rightarrow-\hat{n}$ & $z_{\alpha} \rightarrow i \sigma_{\alpha \beta}^{y} z_{\beta}^{\dagger}$ & $a_{x / y} \rightarrow-a_{x / y}$ \\
\hline \hline
\end{tabular}

TABLE I: Transformations of the Néel and spinor fields on the square lattice under the discrete symmetry generators. Here $i=1,2=x, y$ is a spatial index, and $\epsilon_{i j}=i \sigma_{i j}^{y}$ is the fully antisymmetric rank two tensor. Coördinate transformations of the arguments of the fields have been suppressed.

ing conserved with easy-plane anisotropy. Two currents are also of interest: the spatial current $j_{i}^{z}$ of the conserved Ising (hard-axis) magnetization, and the vorticity three-current $j_{\mu}^{v}$ - the latter being meaningful in the presence of easy plane anisotropy. Finally, we may consider the $\mathrm{CP}^{1}$ gauge three-current $j_{\mu}^{G}=\epsilon_{\mu \nu \lambda} \partial_{\nu} a_{\lambda}$, which in the continuum theory is identified with the topological current $j_{\mu}^{G} \sim \frac{1}{4} \epsilon_{\mu \nu \lambda} \hat{n} \cdot \partial_{\mu} \hat{n} \times \partial_{\nu} \hat{n}$. In a microscopic model, using the transformation properties of these operators, one can construct a (rather complex) superposition of three-spin operators with these same transformation properties. Consider for instance, the time component $j_{0}^{G}=\epsilon_{i j} \partial_{i} a_{j}$. On a square plaquette with central coordinate $r$, number the sites starting at the uppper-left corner of the plaquette and moving clockwise as 1,2,3,4. Then one has

$$
\begin{aligned}
j_{0}^{G}(\mathrm{r}) \sim & (-1)^{\mathrm{r}}\left[\vec{S}_{1} \cdot \vec{S}_{2} \times \vec{S}_{3}-\vec{S}_{2} \cdot \vec{S}_{3} \times \vec{S}_{4}\right. \\
& \left.+\vec{S}_{3} \cdot \vec{S}_{4} \times \vec{S}_{1}-\vec{S}_{4} \cdot \vec{S}_{1} \times \vec{S}_{2}\right],
\end{aligned}
$$

where the $(-1)^{r}$ takes opposite signs on the two sublattices of the dual lattice.

\section{SJ MODELS}

One useful generalization of the SJ model is to allow $\alpha=1 \ldots N$ above, so that $z$ is an $N$-component complex vector of unit magnitude. The $S U(2)$ spin model corresponds to $N=2$. It will be possible to analyse the limits $N=1$ and $N=\infty$. As argued in Section VIIC] the $N=1$ model actually may be realized in a spin- $1 / 2$ model in a staggered Zeeman field. The large- $N$ limit describes ordering transitions of certain $\mathrm{SU}(N)$ quantum antiferromagnets and is less directly physical. Its main utility is its tractability. Similar behavior in both extreme limits - in particular the irrelevance of monopoles in both cases - suggests the same is true for the models with intermediate $N$.

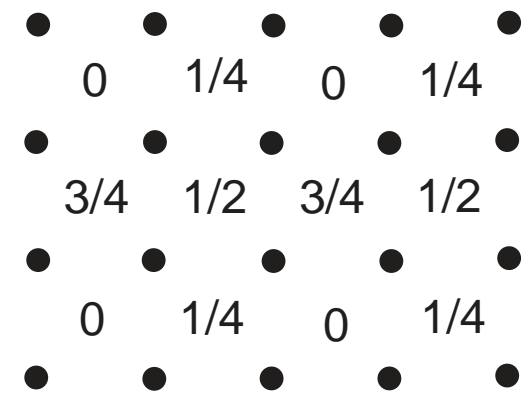

FIG. 4: Specification of the fixed field $\vartheta=-\zeta / 4$. The filled circles are the sites of the direct lattice, and $\vartheta$ resides on the sites of the dual lattice.

\section{A. SJ model at $N=1$}

Consider first $N=1$ where $z_{i} \equiv e^{i \phi_{i}}$ is simply a complex number of unit magnitude. Then

$$
\mathcal{S}_{z}=-2 t \sum_{\ell} \cos (\boldsymbol{\Delta} \phi-\mathbf{a}),
$$

where sum is over the links $\ell$ of the cubic lattice. We indicate spacetime 3 -vectors here in bold face, and the discrete lattice gradient by $\boldsymbol{\Delta}$. As discussed by SJ, this $N=1$ model displays a transition between a Higgs and a translation broken phase. The latter has a four-fold degenerate ground state due to lattice symmetry breaking. Simple symmetry arguments suggest a transition modelled by a $Z_{4}$ clock model - as the four fold anisotropy is irrelevant at the $D=3 \mathrm{XY}$ fixed point, 50 this is in the $3 D \mathrm{XY}$ universality class. SJ also provided numerical evidence supporting this expectation. As shown below, all of this is readily established by a duality transformation of the $N=1$ model.

To dualize the $N=1$ SJ action we use a Villain representation of the $\mathcal{S}_{z}$ term in Eq. (4.1):

$$
\mathcal{S}_{z} \rightarrow \sum_{\ell}\left[\frac{1}{2 \tilde{t}}|\mathbf{j}|^{2}-i \mathbf{j} \cdot(\boldsymbol{\Delta} \phi-\mathbf{a})\right],
$$

The integer valued field $\mathbf{j}$ represents the current of the $z$ field. We also decouple the $S_{a}$ term in Eqn. 3.12 by a Hubbard-Stratanovich field $\mathbf{b}$ to write

$$
\mathcal{S}_{a} \rightarrow \sum\left[\frac{1}{2 K}|\mathbf{b}|^{2}+i \mathbf{b} \cdot(\boldsymbol{\Delta} \times \mathbf{a}-2 \pi \mathbf{q})\right]
$$

Here, and below, the leading sum in the action extends over all sites/links/plaquettes over the cubic lattice, as needed. Performing the sum over the integer field $\mathbf{q}$, we get

$$
\mathbf{b}-\Delta \vartheta=\mathbf{B}
$$

with $\vartheta_{r}=-\zeta_{r} / 4$ (see Fig 4) and $\mathbf{B}$ an integer. If we now integrate over $\phi$, we get the current conservation condition

$$
\Delta \cdot \mathbf{j}=0
$$


This may be solved by writing

$$
\mathbf{j}=\boldsymbol{\Delta} \times \mathbf{A}
$$

with $\mathbf{A}$ an integer. Integrating over the gauge field $\mathbf{a}$, we obtain

$$
\Delta \times \mathbf{B}=\mathbf{j} .
$$

This may be solved by writing

$$
\mathbf{B}=\mathbf{A}+\mathbf{\Delta} \chi
$$

with $\chi$ an integer. The action then reads

$$
\mathcal{S}=\sum\left[\frac{1}{2 \tilde{t}}(\boldsymbol{\Delta} \times \mathbf{A})^{2}+\frac{1}{2 K}(\boldsymbol{\Delta}(\chi+\vartheta)+\mathbf{A})^{2}\right]
$$

The hard integer constraints on $\mathbf{A}, \chi$ may be softened by adding terms

$$
-t \cos (2 \pi \mathbf{A})-\sum_{n} \lambda_{n} \cos (2 \pi n \chi)
$$

We may now shift $\chi \rightarrow \tilde{\chi}=\chi+\vartheta, \mathbf{A} \rightarrow \mathbf{A}^{\prime}=\mathbf{A}+\boldsymbol{\Delta} \tilde{\chi}$. The $\mathbf{A}^{\prime}$ field is massive and may be integrated out. The remaining action for the $\tilde{\chi}$ reads

$$
\mathcal{S}=\sum\left[-t \cos (2 \pi \Delta \tilde{\chi})-\sum_{n} \lambda_{n} \cos [2 \pi n(\tilde{\chi}-\vartheta)]\right]
$$

This describes an XY model with various $n$-fold anisotropy terms of strengths $\lambda_{n}$ The shift by $\vartheta$ leads to rapid spatial oscillations of these anisotropy terms unless $n=0(\bmod 4)$. Near the critical point in the continuum limit, the leading non-vanishing anisotropy term is at $n=4$. The critical properties are therefore that of an XY model with four-fold anisotropy $\lambda_{4}$. The latter has a scaling dimension $\Delta_{4}>3$, which renders it irrelevant at the $D=3 \mathrm{XY}$ critical point 50

An overly cautious reader may object that uncontrolled approximations have been made in softening the integer constraints on the $\mathbf{A}, \chi$ fields. However, all manipulations up to Eq. 4.9 are exact, and from this point an exact world-line representation may be obtained by implementing the integer constraints using the Poisson resummation formula. The latter representation clearly describes charged relativistic particles for which charge non-conservation events oscillate spatially unless the charge is changed in multiples of four. On universality grounds, one expects this model to be in the same universality class as an XY model with 4-fold anisotropy. Though we will not pursue it, a similar exact duality can be performed on the $N=2$ SJ model in a world-line representation, and may be used to somewhat more rigorously argue for self-duality of the critical theory in this case.

The results above can be interpreted physically as follows. Let us first consider the vortices in the $z$ condensate. These will carry gauge flux that is quantized in units of $2 \pi$. Such a $2 \pi$ flux can end at a space-time monopole. Hence monopoles act as sources of the vortices of the $z$ field. The Berry phases imply that these monopole events are quadrupled so that only processes where four vortices disappear (or are created) together are important in the continuum limit. Now if we forbid monopoles by hand, then the usual duality arguments map the model to a global XY model in terms of the vortex fields. The dual global U(1) symmetry of this XY model is precisely associated with conservation of vorticity. Including monopoles (which act as sources for 4 vortices) introduces a four-fold anisotropy on this global $\mathrm{XY}$ model. Such an anisotropy is irrelevant at the $3 D \mathrm{XY}$ critical fixed point. Thus monopole events are again irrelevant and (in the original representation) a theory where the $z$ boson is coupled to a non-compact $\mathrm{U}(1)$ gauge field describes the transition.

\section{B. SJ model at large $N$}

Now let us consider $N$ large. In the limit $N \rightarrow \infty$ the gauge field is non-fluctuating and can be taken as a classical 'background' in which the $z$ particles move. The minimum energy saddle point corresponds to $a_{\mu}=0$ (up to gauge rotation). The $z$ bosons are gapped and free in the paramagnetic state, while they are condensed in the ordered state. Now consider the nature of both states, and the transition, upon including fluctuations in a $1 / N$ expansion. It is useful to discuss the effects of instantons separately from other fluctuations. Ignoring instantons, the $1 / N$ expansion proceeds along standard lines. In the ordered state, the gauge fields acquire a mass by the usual Anderson-Higgs mechanism. The gauge flux is quantized in units of $2 \pi$ - the associated point defects are the large- $N$ avatars of the skyrmion described previously directly at $N=2$. However, on the paramagnetic side the gauge fields are gapless and describe a 'photon' which disperses linearly at low energies. The transition is described by a field theory of $z$ bosons coupled minimally to a non-compact $\mathrm{U}(1)$ gauge field. This transition is second order with critical exponents that evolve continuously from their values at $N=\infty$. In particular, consider the gauge invariant physical spin operator (which is the appropriate generalization to large- $N$ of the familiar Néel order parameter at $N=2$ ). This is bilinear in the $z$ fields. At $N=\infty$, the spin operator therefore has a large anomoulous dimension $\eta=1$. This will acquire (calculable) corrections 52.53 of $\mathcal{O}(1 / N)$ upon considering finite but large $N$. Hence $\eta$ will be large for large but finite $N$.

Now consider including instantons. It is important to realize that the entire gauge action is of order $N$ in this theory. Consequently, 35 the 'bare' instanton core action, obtained by integrating out the $z$ fields in the presence of a background instanton configuration of the gauge fields in space-time, is of order $N$. Thus the bare instanton fugacity is small (exponentially small in $N$ ). In the or- 
dered state, the inclusion of instanton events means that point defects with quantized $2 \pi$ flux are no longer stable. The physics in the paramagnetic state is more interesting. Here the instantons proliferate and lead to confinement of the gapped $z$ bosons. Furthermore, the gapless photon (present in the non-compact model) is rendered unstable. The Haldane Berry phases associated with the instantons lead to lattice symmetry breaking. As explained in Section III this follows from the observation that the instanton operators transform non-trivially under lattice symmetries. Hence if they acquire an expectation value, lattice symmetry is broken.

Now let us consider the effect of instantons at the transition. ¿From the discussion in preceding sections, it is clear that the crucial question is whether the fourmonopole event is relevant/irrelevant at the fixed point of this non-compact model. The scaling dimension of the $p$-monopole operator in this model was computed by Murthy and Sachdev ${ }^{35}$. For $p=4$, their results give a scaling dimension $\propto N$. Hence the instantons are strongly irrelevant ${ }^{37}$ for large $N$.

This then implies that the critical point of the noncompact theory is stable to inclusion of instanton events, even though the states on both sides of the critical point are qualitatively changed. In particular, consider approaching the transition from the paramagnetic side. The proliferation of instantons in the paramagnetic state had two effects - to confine the spinons and to produce VBS order. The irrelevance of the instantons at the critical fixed point implies that both the VBS order and the spinon confinement disappear at the transition. We note that as the bare instanton fugacity is exponentially small in $N$, this perturbative analysis of their relevance/irrelevance is sufficient to determine the nature of the transition. In particular the alternate possibility that there is a coexistence region with width shrinking to zero as $N \rightarrow \infty$ appears unlikely at large- $N$.

It is also useful to interpret the results above in the context of other recent discussions of instantons in the literature 28.29.30.31. The strategy of these, and other works, is to integrate out the $z$ bosons, and to work with an effective action for the gauge field. This action will be of order $N$. Consequently, it seems reasonable to assume that the gauge field dynamics is described to leading order in $1 / N$ by a Gaussian action (this is equivalent to the RPA approximation), and to address issues of instanton physics within this Gaussian gauge action. Such an approach will correctly describe the qualitative physics of the paramagnetic state. For the critical point itself, the form of the Gaussian gauge action is determined by scaling to be ${ }^{28.29}$ (see also Eq. (8.2)

$$
\mathcal{S}_{G}=\int \frac{d^{3} K}{(2 \pi)^{3}} N \sigma_{0}|K|\left|\mathbf{a}_{T}(\mathbf{K})\right|^{2}
$$

where $\mathbf{K}$ is the Euclidean 3 -momentum, $\mathbf{a}_{T}$ refers to the transverse part of the gauge field, and $\sigma_{0}$ is a universal constant associated with the universal critical conductivity of the $z$ bosons at the transition at $N=\infty$. Note that this action is more singular than the usual 'Maxwell' action - this originates in the integration over the massless critical modes of the $z_{\alpha}$ fields. The action for a single instanton can be calculated within this Gaussian approximation, and is of order $N \ln L$ where $L$ is the system size. This suggests that instanton-anti-instanton pairs interact logarithmically with each other. It also suggests that the effect of instantons could be captured by studying a classical three dimensional Coulomb gas of instantons with pairwise logarithmic interactions. If this gas is in a plasma phase, free instantons have proliferated. On the other hand, one might also conceive a different phase where instanton-anti-instanton pairs are strongly bound to each other. For the classical $3 D$ Coulomb gas, examination of this issue $\frac{29.30}{2}$ has led to the conclusion that the logarithmic interaction is screened at long length scales into a short-ranged interaction, by bound instanton pairs at shorter scales (however, it was noted ${ }^{29.30}$ that the screening could fail at fine-tuned critical points). This screening then forces proliferation of free instantons, so that the Coulomb gas is in a plasma phase. How are we to reconcile this apparently general conclusion with our claim that the instantons are suppressed at the Néel-VBS critical point?

This conundrum is resolved as follows. The Gaussian 'RPA' action does not properly account for the effects of highly non-linear perurbations such as instantons. This is already clear from the results of Ref. 35 . Within the Gaussian theory, the action of a strength $p$ instanton scales with $p$ as $p^{2}$. This would imply that the scaling dimension of the $p$ instanton operator scale as $p^{2}$ - this disagrees with results of Ref. 35 , which obtained a highly non-trivial dependence on $p$. In other words, even in the large- $N$ limit, the Gaussian action is not sufficient to correctly calculate the scaling dimension of the instantons: the non-linear terms in the gauge action all contribute in determining the instanton action ${ }^{54}$ even at $N=\infty$. More significantly, we can likewise conclude that the RPA treatment of instanton interactions by a simple pairwise interaction is inadequate. The true instanton gas (even in the large- $N$ limit) has a rather specific structure of higher order interactions, some of whose features are universally determined by the fact that they arose from integrating out particular gapless critical modes. If we attempt to compute the screening of instanton interactions by integrating out bound instanton-anti-instanton pairs, effects which renormalize the screening length are intricately intertwined with those that shift the position of the critical point between the magnetic and paramagnetic phases. Indeed, fine-tuning to be at the critical point between the magnetic and paramagnetic phases is all that is needed to also suppress the instanton plasma phase, and the 'nä̈ve' conclusion that the instantons are irrelevant at this critical point ${ }^{35}$ is correct. Appendix $\mathrm{F}$ considers a specific toy model for which these arguments can be demonstrated explicitly.

As we noted at the end of Section II the above reasoning may also apply to fermionic models which have a 
line of critical points 29.31 : in this case, the suppression of instantons may occur along the entire line, and not just at an isolated point.

\section{SPIN MODELS WITH EASY PLANE ANISOTROPY}

An alternate and particularly fruitful deformation of the model is provided by the situation where there is some easy-plane anisotropy on the underlying $S U(2)$ spin model. Such an anisotropy tends to orient the spins preferentially perpendicular to the $z$-axis in spin space. Indeed precisely such an easy plane spin- $1 / 2$ model with both two particle and four-particle ring exchanges has recently been studied numerically ${ }^{5}$. A direct transition between Néel and valence bond solid phases was found.

Consider first the fate of the global symmetries in the presence of easy plane anisotropy. A U(1) subgroup of symmetry of spin rotations about the $z$-axis of spin still survives. In addition there are a number of discrete symmetries. Either under a unit translation or time reversal (see Table \), the Néel vector changes sign

$$
\hat{n}_{r} \rightarrow-\hat{n}_{r} .
$$

This may be combined with a $\mathrm{U}(1)$ spin rotation in the $\mathrm{XY}$-plane which restores the sign of $n^{x}, n^{y}$ to simply change the sign of $n^{z}$ alone. Thus $n^{z} \rightarrow-n^{z}$ is a discrete symmetry in the easy-plane case.

Easy plane anisotropy is readily incorporated into the non-linear sigma model description in Eqs. (2.1) or (3.1) as a term

$$
\mathcal{S}_{\mathrm{ep}}=-\int d \tau d^{2} x w\left(n^{z}\right)^{2}
$$

with $w<0$ (this is clearly related to Eq. (1.8)). The global U(1) symmetry simply corresponds to a uniform rotation of all the $\hat{n}$ vectors about the $z$-axis.

\section{A. Semi-classical analysis}

Let us first think classically about this easy plane model. By classical we mean to focus on time independent configurations of the $\hat{n}$-field and to ignore the Berry phase effects. The classical ground state simply consists of letting $\hat{n}$ be independent of position and lie entirely in the spin XY plane. Topological defects in this ground state will play an important role. With the easy plane anisotropy, these are simply vortices in the field $n^{+}=n_{x}+i n_{y}$. More precisely, on going around a large loop containing a vortex the phase of $n^{+}$winds around by $2 \pi m$ with $m$ an integer.

What is the nature of the core of these vortices? In the core the XY order will be suppressed and the $\hat{n}$ vector will point along the $\pm \hat{z}$ direction. In terms of the microscopic spin model, this corresponds to a non-zero
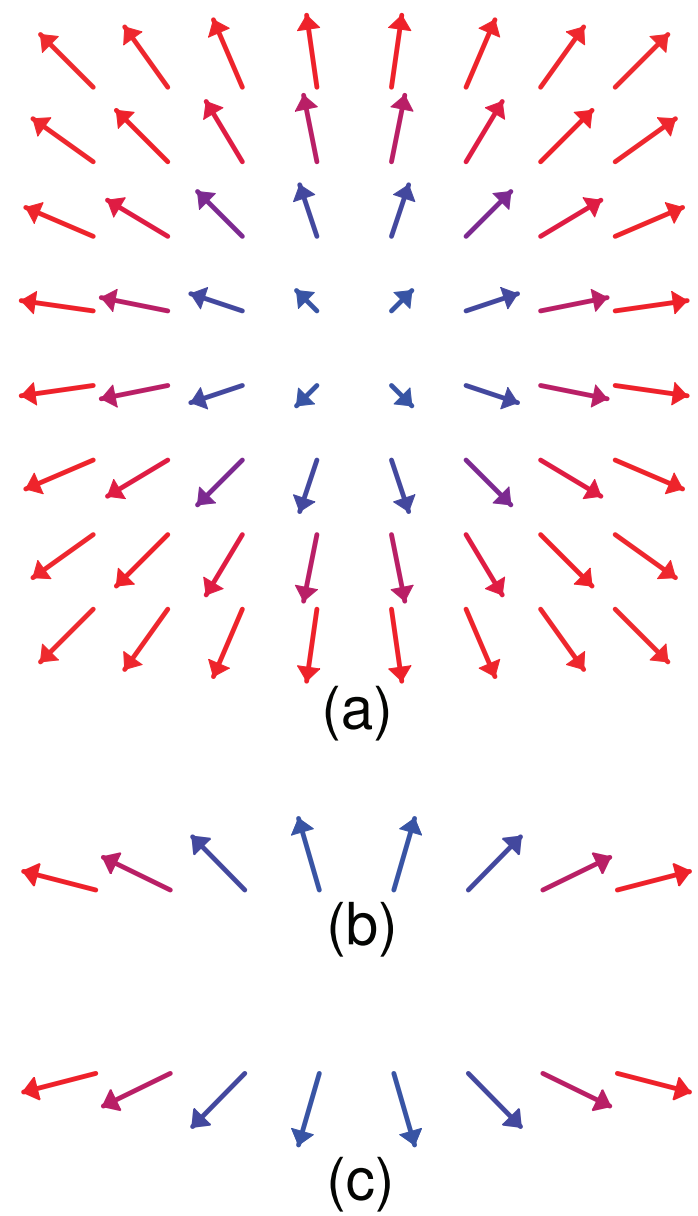

FIG. 5: The 'meron' vortices in the easy plane case. There are two such vortices, $\psi_{1,2}$, and $\psi_{1}$ is represented in (a) and (b), while $\psi_{2}$ is represented by (a) and (c), following the conventions of Fig [2] The $\psi_{1}$ meron above has $\hat{n}(r=0)=(0,0,1)$ and $\hat{n}(|r| \rightarrow \infty)=(x, y, 0) /|r|$; the $\psi_{2}$ meron has $\hat{n}(r=0)=$ $(0,0,-1)$ and the same limit as $|r| \rightarrow \infty$. Each meron above is 'half' the skyrmion in Fig 2 this is evident from a comparison of (b) and (c) above with Fig [2 b. Similarly, one can observe that a composite of $\psi_{1}$ and $\psi_{2}^{*}$ makes one skyrmion.

staggered magnetization of the $z$ component of the spin in the core region. Thus at the classical level there are two kinds of vortices depending on the direction of the $\hat{n}$ vector at the core (see Fig. 55). Note that either kind of vortex breaks the Ising-like $n^{z} \rightarrow-n^{z}$ symmetry at the core.

Clearly this breaking of the Ising symmetry is an artifact of the classical limit - once quantum effects are included, the two broken symmetry cores will be able to tunnel into each other and there will be no true broken Ising symmetry in the core. This tunneling is often called an 'instanton' process that connects two classically degenerate states.

Interestingly such an instanton event is physically the easy-plane avatar of the space-time monopole described above for the fully isotropic model. This may be seen pictorially. Pictorially each classical vortex really represents 
half of a skyrmion configuration. Such half-skyrmions are known as merons. As shown in Fig. 5 the tunneling process between the two merons is equivalent to creating a full skyrmion. This is precisely the monopole event.

Now the results of Haldane imply once again that (in the continuum) monopole events are quadrupled. Thus the only tunneling processes that survive in the continuum limit are those in which four merons with core spins along one direction come together and collectively flip the orientation of their core spins to produce four merons of the opposite kind.

\section{B. Easy plane in the $\mathrm{CP}^{1}$ representation}

It is extremely useful to also consider easy plane anisotropy in the framework of the $\mathrm{CP}^{1}$ representation. In this representation, the easy plane anisotropy was already presented in Eq. (1.8). Let us first translate the above classical thinking into the $\mathrm{CP}^{1}$ representation. Suppose for this purpose $w$ is negative but small, so that states with $\left|z_{1}\right|=\left|z_{2}\right|$ are favored but not rigidly enforced. Clearly, the preferred uniform classical ordered states satisfy

$$
\left|\left\langle z_{1}\right\rangle\right|=\left|\left\langle z_{2}\right\rangle\right| \neq 0,
$$

so that $n^{+}=z_{1}^{*} z_{2}$ is ordered and there is no average value of $n^{z}=\left|z_{1}\right|^{2}-\left|z_{2}\right|^{2}$. Now consider vortex configurations. Clearly a full $2 \pi$ vortex in $n^{+}$can be achieved by either having a $2 \pi$ vortex in $z_{1}$ and not in $z_{2}$ or a $2 \pi$ anti-vortex in $z_{2}$ and no vortex in $z_{1}$. Far from the vortex core both fields will have equal amplitude, but in the first choice the amplitude of the $z_{1}$ condensate will be suppressed at the core but $\left\langle z_{2}\right\rangle$ will be unaffected. Consequently $n^{z}=\left|z_{1}\right|^{2}-\left|z_{2}\right|^{2}$ will be non-zero and negative in the core. The other choice also leads to non-zero $n^{z}$ which will now be positive. Thus we may identify the two kinds of meron vortices with $2 \pi$ vortices in the spinon fields $z_{1,2}$ respectively.

To explore this analytically, we consider the behavior now deep in the easy plane limit, in which $n^{z}=$ $\left|z_{i 1}\right|^{2}-\left|z_{i 2}\right|^{2} \approx 0$. Together with the $\mathrm{CP}^{1}$ constraint $\left|z_{i 1}\right|^{2}+\left|z_{i 2}\right|^{2}=1$, this implies fixed magnitude for each component of $z_{i}$, so we may write

$$
z_{i \alpha} \sim \frac{1}{\sqrt{2}} e^{i \phi_{i \alpha}}
$$

where $\phi_{i \alpha} \in[0,2 \pi)$ is the phase of the spinon field. The "kinetic" term of the SJ model action in Eq. (3.11) is then modified to

$$
\mathcal{S}_{z}=-t \sum_{\ell, \alpha} \cos \left(\Delta \phi_{\alpha}-\mathbf{a}\right),
$$

with the other terms $\left(\mathcal{S}_{a}, \mathcal{S}_{B}\right)$ given as before in Eqs. (3.12) and (3.13).

It is very useful both for further insight and for concrete calculations to explore a 'dual' representation which focuses on the meron vortex fields. Although the form of the dual action is dictated completely by the general considerations of the previous subsection, we provide an explicit derivation in Appendix B by proceeding as in Section IVA with the duality transformation. We obtain the dual action $\mathcal{S}_{\text {dual }}=\int d^{2} r d \tau \mathcal{L}_{\text {dual }}$ with

$$
\begin{aligned}
& \mathcal{L}_{\text {dual }}=\sum_{a=1,2}\left|\left(\partial_{\mu}-i A_{\mu}\right) \psi_{a}\right|^{2}+s_{d}|\psi|^{2}+u_{d}\left(|\psi|^{2}\right)^{2} \\
& +w_{d}\left|\psi_{1}\right|^{2}\left|\psi_{2}\right|^{2}+\kappa_{d}\left(\epsilon_{\mu \nu \kappa} \partial_{\nu} A_{\kappa}\right)^{2}-\lambda \operatorname{Re}\left[\left(\psi_{1}^{*} \psi_{2}\right)^{4}\right] .
\end{aligned}
$$

We have used the short-hand notation $|\psi|^{2} \equiv\left|\psi_{1}\right|^{2}+$ $\left|\psi_{2}\right|^{2}$. Here $\psi_{1,2}$ denote quantum fields that destroy meron vortices whose core points in the up direction for $\psi_{1}$ and down for $\psi_{2}$.

We now show how the dual action of Eq. (5.6) can be understood entirely on general grounds. As usual in dual theories, the net vorticity is conserved, corresponding to the overall $\mathrm{U}(1)$ symmetry of Eq. (5.6). This symmetry is gauged by the non-compact vector potential $A_{\mu}$, as usual in dual descriptions of two dimensional bosonic systems. Physically, the gauge field is required to embody spin $\left(S^{z}\right)$ conservation of the original model, $j_{\mu}=\epsilon_{\mu \nu \lambda} \partial_{\nu} A_{\lambda} / \pi$ being the 3 -current of $S^{z}$. Hence the dual magnetic and electric fields correspond to the spin density and spin current, respectively. Minimal coupling of the vortex fields to $A_{\mu}$ also gives them proper logarithmic interactions and magnus force dynamics.

Clearly under the discrete Ising-like $n^{z} \rightarrow-n^{z}$ symmetry, the two vortices get interchanged, i.e $\psi_{1} \rightarrow \psi_{2}$ and vice-versa. The dual action must therefore be invariant under interchange of 1 and 2 labels.

Finally, if monopole events were to be completely ignored (i.e disallowed by hand) the total skyrmion number must be conserved. As is apparent from Fig [5 a composite of a $\psi_{1}$ vortex and a $\psi_{2}^{*}$ antivortex is precisely a skyrmion configuration of the $\hat{n}$ field. Thus we may view skyrmion number conservation as the conservation of the difference of the total number of either species of vortices. This implies the global U(1) symmetry

$$
\begin{aligned}
& \psi_{1} \rightarrow \psi_{1} \exp (i \varrho) \\
& \psi_{2} \rightarrow \psi_{2} \exp (-i \varrho)
\end{aligned}
$$

where $\varrho$ is a constant.

As discussed at length above, monopole events destroy the conservation of skyrmion number, and hence this dual global U(1) symmetry. However as the monopoles are effectively quadrupled due to the Berry phase terms, skyrmion number is still conserved $(\bmod 4)$. Thus the dual global U(1) symmetry must be broken down to $Z_{4}$.

The dual Lagrangian $\mathcal{L}_{\text {dual }}$ in Eq. (5.6) is the simplest one that is consistent with all these requirements. In particular, we note that at $\lambda=0$ the dual global $\mathrm{U}(1)$ transformation in Eq. (5.7) leaves the Lagrangian invariant. The $\lambda$ term breaks this down to $Z_{4}$ as required. Thus we may identify $\lambda$ as the fugacity of the (quadrupled) monopole tunneling events, $\lambda \sim \lambda_{4}$ in Eq. (1.9). 
Actually this action was first derived by completely different means in Refs. 48 and 38 . The discussion above is however more directly physical, and gives an interpretation of the $\lambda$ term and of the other symmetries of this dual action.

An important coupling constant in the above dual action is $w_{d}$, which appears as a sort of "anisotropy". The exact lattice duality in the appendix in fact leads to a "hard spin" (rotor) model in which $\left|\psi_{1}\right|=\left|\psi_{2}\right|=1$. The above continuum theory is arrived by "softening" this constraint. However, it is clear that the appropriate sign of $w_{d}$ to connect microscopically to the original SJ model is $w_{d}<0$ (and large). While the above symmetry arguments do not specify this sign, the model with $w_{d}>0$ presumably corresponds to rather different physics, and has no clear connection to the original SJ model. See the discussion in Sec. IXD for a possible physical application of this case.

Note that apart from the $\lambda$ term, Eq. (5.6) has exactly the same structure as the continuum $\mathrm{CP}^{1}$ theory $\mathcal{L}_{z}$ in Eq. (1.7) in the presence of the easy plane anisotropy Eqn. (1.8). As we will discuss below, the $\lambda$ term (which represents the quadrupled monopole tunneling events) are irrelevant at the QCP: consequently, the QCP has a self-dual structure.

We should further note that there is no connection between $w_{d}$ in the vortex action and the analogous parameter $w$ in the continuum limit of the SJ model. The latter is clearly simply related to the physical spin anisotropy, corresponding for $w<0$ to easy-plane and for $w>0$ to easy-axis anisotropy. The point $w=0$ describes the $\mathrm{SU}(2)$ invariant magnetic QCP. This does not correspond to $w_{d}=0$ in the dual theory. Indeed, there may be no dual theory whatsoever for any but the easy plane case (though see Sec. IXD).

A list of the representation of physical operators of interest in the original and dual representations is given in Table【 In the dual vortex theory, the XY ordered phase is simply characterized as a dual 'paramagnet' where both the $\psi_{1,2}$ fields are gapped. On the other hand, true spin paramagnetic phases correspond to condensates of the fields $\psi_{1,2}$, which break the dual gauge symmetry. In particular if both $\psi_{1}$ and $\psi_{2}$ condense with equal amplitude $\left\langle\psi_{1}\right\rangle=\left\langle\psi_{2}\right\rangle \neq 0$, then a paramagnetic phase where the global Ising symmetry is preserved results. Note the strong similarity between the description of the phases in this dual theory with that in terms of the spinon fields of the $\mathrm{CP}^{1}$ representation if we interchange the role of the $\mathrm{XY}$ ordered and paramagnetic phases. This is a symptom of an exact duality between the two descriptions that obtains close to the transition. At this point, the two descriptions do not appear wholly identical, due to the $Z_{4}$ symmetry-breaking term $\lambda$ not present in the $\mathrm{CP}^{1}$ theory, and the compactness of the $\mathrm{CP}^{1}$ gauge field not present in the dual one. As argued above, the two differences represent one and the same physics, since the vortex tunneling events generated by $\lambda$ represent exactly the non-oscillating four-monopole events allowed in the

\begin{tabular}{l|c|c}
\hline \hline Field & $\mathrm{CP}^{1}$ & dual \\
\hline$N^{+}$ & $z^{\dagger} \sigma^{+} z$ & $e^{i \int E_{j} \mathcal{A}_{j}}$ \\
$\psi_{\mathrm{VBS}}$ & $e^{i \int e_{j} \mathcal{A}_{j}}$ & $\psi^{\dagger} \sigma^{+} \psi$ \\
$N^{z}$ & $z^{\dagger} \sigma^{z} z$ & $\psi^{\dagger} \sigma^{z} \psi$ \\
$M^{+}$ & $i z^{\dagger} \sigma^{+}\left(\overleftrightarrow{\partial}_{0}-i a_{0}\right) z$ & $e^{i \int E_{j} \mathcal{A}_{j}} \psi^{\dagger} \sigma^{z} \psi$ \\
$M^{z}$ & $i z^{\dagger} \sigma^{z}\left(\overleftrightarrow{\partial}_{0}-i a_{0}\right) z$ & $\epsilon_{i j} \partial_{i} A_{j} / \pi$ \\
$j_{i}^{z}$ & $i z^{\dagger} \sigma^{z}\left(\overleftrightarrow{\partial}_{i}-i a_{i}\right) z$ & $\epsilon_{i j}\left(\partial_{0} A_{j}-\partial_{j} A_{0}\right) / \pi$ \\
$j_{\mu}^{v}$ & $\operatorname{vorticity}$ & $i \psi^{\dagger}\left(\overleftrightarrow{\partial}_{\mu}-i A_{\mu}\right) \psi$ \\
$j_{\mu}^{G}$ & $\epsilon_{\mu \nu \lambda} \partial_{\mu} a_{\lambda} / \pi$ & $i \psi^{\dagger} \sigma^{z}\left(\stackrel{\partial}{\partial}_{\mu}-i A_{\mu}\right) \psi$ \\
\hline \hline
\end{tabular}

TABLE II: Operators in the easy plane $\mathrm{CP}^{1}$ (column 2) and dual (column 3) representations corresponding to physical operators (column 1), in the notations of Sec. III Here we have introduced the classical gauge field configuration for a unit point flux, with $\epsilon_{i j} \partial_{i} \mathcal{A}_{j}(x)=2 \pi \delta^{2}(x)$. The symbol $e_{j}$ represents the $j$ - componenet of the electric field operator that corresponds to the gauge field in the $C P^{1}$ representation. We have also used the symbol $\overleftrightarrow{\partial}_{\mu}$, defined by $f \overleftrightarrow{\partial}_{\mu} g=\frac{1}{2}\left[f \partial_{\mu} g-\left(\partial_{\mu} f\right) g\right]$. The symbol $j_{i}^{z}$ is the current of conserved magnetization, while $j_{\mu}^{v}$ and $j_{\mu}^{G}$ are the threecurrents of vorticity and gauge flux, respectively.

SJ model. In the next section, we will argue that these events are irrelevant in the scaling limit near the QCP, making the duality between the two descriptions complete.

As indicated in Table III the combination $\psi_{\text {VBS }} \sim$ $\psi_{1}^{*} \psi_{2}$ serves as the order parameter for the translational symmetry broken VBS ground state. This may be seen from the analysis of Refs. 38 48. Alternately this may be seen by the identification described in Sec. III of the skyrmion creation operator with the order parameter for translation symmetry breaking. Such a condensate of $\psi_{1,2}$ breaks the global $Z_{4}$ symmetry of the action in Eq. (5.6). The preferred phase of $\psi_{\mathrm{VBS}}$ depends on the sign of $\lambda$, the two inequivalent sets of preferred directions corresponding to columnar and plaquette patterns of translational symmetry breaking.

\section{PHASE TRANSITIONS}

\section{A. Easy plane limit}

Consider the dual vortex action in Eq. (5.6). In mean field theory the transition happens when the parameter $s_{d}$ becomes smaller than zero and can clearly be second order. Fluctuation effects will modify the mean field behavior in important ways. Consider first the properties of the transition when $\lambda=0$, i.e in the absence of instanton events. The resulting model has recently been studied in Ref. 21. Remarkably, as argued there, the model has the property of being self-dual - the ordered and paramagnetic phases get interchanged under the duality transformation. To understand this first note that in the $\lambda=0$ limit, the dual action Eq. (5.6) has pre- 
cisely the same structure as an easy-plane $\mathrm{CP}^{1}$ model with a non-compact $\mathrm{U}(1)$ gauge field (as in Eq. (1.7)). As this same limit actually corresponds to disallowing all monopole events, in the spinon description we must work with a non-compact gauge field. Then the exact same field theory obtains both in terms of the spinon fields $z$ (in Eq. (1.7)) and in terms of the meron vortices $\psi$ in the easy plane limit (in Eq. (5.6)) and ignoring instantons.

It was established in Ref. 21 via numerical Monte Carlo simulations that a continuous ordering transition exists in this model with the non compact gauge field. The fixed point controlling this transition in this limit is therefore described by a self-dual field theory. Note that in either representation the natural fields of the theory are not those associated with the 'physical' boson operator (either $n^{+}$or the skyrmion creation operator). Rather the theory is expressed most simply in terms of 'fractionalized' fields - namely the spinons or the meron vortices. In particular, the physical $n^{+}$field is a composite of two spinon fields and likewise the skyrmion field is a composite of the two meron fields.

Let us now imagine including instanton events. This is most easily accessed in the vortex representation where it simply amounts to letting $\lambda \neq 0$. This is the main advantage of the dual representation - the non-trivial nonlocal effect of instantons is represented as a simple local perturbation in the dual theory. We may now address the question of relevance/irrelevance of instantons at the $\lambda=0$ fixed point. This is determined by the scaling dimension $\Delta$ of the $\left(\psi_{1}^{*} \psi_{2}\right)^{4}=\psi_{\text {VBS }}^{(4)}$ operator, in principle determined from the two-point correlation function of this operator in the (non-trivial) theory with $\lambda=0$ :

$$
\left\langle\psi_{\mathrm{VBS}}^{(4)}(x) \psi_{\mathrm{VBS}}^{(4) *}\left(x^{\prime}\right)\right\rangle_{\lambda=0} \sim \frac{1}{\left|x-x^{\prime}\right|^{2 \Delta}},
$$

where $x, x^{\prime}$ are space-time coordinates. Hence $\Delta$ is determined by the correlations of the fourth power of the physical VBS order parameter, and one requires $\Delta>D=3$ for irrelevance. Being self-dual, the same anomalous dimension should be ascribed to the correlations of the physical boson (XY order parameter). The $\lambda=0$ critical fixed point describes an XY ordering transition where the physical boson field is a composite of the fundamental fields of the theory. We therefore expect that correlators of the physical boson (and its various powers) will decay with an anomalous dimension that is larger than the corresponding one for the ordinary $\mathrm{XY}$ transition in $D=2+1$ dimensions. Now for the usual XY fixed point four-fold symmetry breaking perturbations are known to be irrelevant, i.e. have a scaling dimension $\Delta_{4}>3$. This then implies that a small $\lambda$ will be irrelevant by power counting at the $\lambda=0$ fixed point of the present model as well (see also Appendix [C).

This latter expectation can be checked in an appropriate large $N$ generalization. In particular, consider the non-compact gauge theory with Lagrangian

$$
\begin{aligned}
\mathcal{L} & =\sum_{i=1}^{2 N}\left[\left|\left(\partial_{\mu}-i A_{\mu}\right) \psi_{i}\right|^{2}+r\left|\psi_{i}\right|^{2}+u\left|\psi_{i}\right|^{4}\right] \\
& +\kappa N\left(\epsilon_{\mu \nu \lambda} \partial_{\nu} A_{\lambda}\right)^{2}-\lambda \sum_{i=1}^{N}\left[\left(\psi_{i}^{*} \psi_{i+N}\right)^{4}+\text { c.c. }\right](6.2)
\end{aligned}
$$

where the $U(1) \times U(1)$ symmetry (for $\lambda=0$ ) of the dual action has been elevated to a $U(1)^{2 N}$ invariance (under independent phase rotations of each $\psi_{i}$ field). Of this, only the single $U(1)$ subgroup of identical rotations of all $2 N$ fields has been gauged with the non-compact gauge field $A_{\mu}$. The $\lambda$ term breaks the $U(1)^{2 N}$ symmetry to $U(1)^{N}$, of which $N-1$ are global, and the single gauge $\mathrm{U}(1)$ is preserved. In addition, there is a residual global $Z_{4}^{N}$ symmetry under $\psi_{j} \rightarrow e^{i n_{j} \pi / 4} \psi_{j}, \psi_{j+N} \rightarrow$ $e^{-i n_{j} \pi / 4} \psi_{j+N}$, with $n_{j} \in\{0,1,2,3\}$, for $j=1 \ldots N$.

In the $N=\infty$ limit, the theory may be analyzed by saddle point methods. In particular, consider for simplicity the partition function with $\lambda=0$, which may be formally written

$$
\begin{aligned}
\mathcal{Z}=\int[d A] \exp & \left\{-N\left[2 \mathcal{S}_{X Y}^{\mathrm{eff}}\left(A_{\mu}\right)\right.\right. \\
+ & \left.\left.\int d^{2} r d \tau \kappa\left(\epsilon_{\mu \nu \lambda} \partial_{\nu} A_{\lambda}\right)^{2}\right]\right\},
\end{aligned}
$$

where

$$
\begin{aligned}
\mathcal{S}_{X Y}^{\mathrm{eff}}=- & \ln \int[d \psi] \exp \left\{-\int d^{2} r d \tau[\right. \\
& \left.\left.\left|\left(\partial_{\mu}-i A_{\mu}\right) \psi\right|^{2}+r|\psi|^{2}+u|\psi|^{4}\right]\right\}
\end{aligned}
$$

is the effective action of the $D=3 \mathrm{XY}$ model as a functional of $A_{\mu}$. Formally, at $N=\infty$, from Eq. 6.3 a saddle point approximation in $A_{\mu}$ is justified, with the saddle point value being zero, $A_{\mu}^{*}=0$. The relevance of $\lambda$ is then determined by the two-point function of $\psi_{V B S ; N}^{(4)}=\sum_{i=1}^{N}\left(\psi_{i}^{*} \psi_{i+N}\right)^{4}$ in the saddle point theory with $A_{\mu}=0$. Since in this theory the $\psi_{i}$ are decoupled $\mathrm{XY}$ fields (fluctuating according to the non-trivial $3 D$ $\mathrm{XY}$ fixed point), one has then

$$
\begin{aligned}
& \left\langle\psi_{\mathrm{VBS} ; N}^{(4)}(x) \psi_{\mathrm{VBS} ; N}^{(4) *}\left(x^{\prime}\right)\right\rangle_{\lambda=0} \\
& \sim N\left|\left\langle\left(\psi^{*}(x)\right)^{4}\left(\psi\left(x^{\prime}\right)\right)^{4}\right\rangle_{3 D X Y}\right|^{2} \sim \frac{N}{\left|x-x^{\prime}\right|^{4 \Delta_{4}}} .
\end{aligned}
$$

The final expression obtains since the expectation value in the second line is none other but the two-point function of the four-fold symmetry breaking field at the $D=3 \mathrm{XY}$ fixed point. Hence, one has $\Delta=2 \Delta_{4}$, implying $\Delta>6$ since $\Delta_{4}>3$. Thus in this limit the "monopole" (symmetry breaking) terms are strongly irrelevant. We note in passing that the irrelevance of $\lambda$ can also be established 
by working for $\lambda \neq 0$, and taking the large $N$ limit. The saddle point remains at $A_{\mu}=0$, and irrelevance follows simply from the irrelevance of "bi-quartic" coupling between two $3 D \mathrm{XY}$ models by their four-fold symmetry breaking fields.

We conclude that a direct second order transition with irrelevant instanton tunneling events is possible in this easy plane case. Note the crucial role played by the Berry phase term for the instantons in reaching this conclusion. Indeed it was the Berry phases that forced quadrupling of instantons thereby increasing their scaling dimension and making it possible for them to be irrelevant.

While the $\lambda$ term may be irrelevant at the critical fixed point it is clearly very important in deciding the fate of either phase. In particular in the paramagnetic phase it picks out the particular pattern of translation symmetry breaking (columnar versus plaquette) and forces linear confinement of spinons. In critical phenomena parlance, it may be described as a dangerously irrelevant perturbation.

\section{B. Isotropic magnets}

In the context of the SJ models, the results of previous sections show that the $N=1, N=\infty$, and easy plane $N=2$ models all provide the same picture. A direct second order transition between the $z$ condensed and VBS phases is possible with a 'deconfined' critical point. Right at this point, monopole tunneling events become irrelevant and spinon degrees of freedom emerge as the natural fields of the critical theory. This provides strong evidence that the same thing happens for the $S U(2)$ symmetric model (i.e at $N=2$ ).

What then is the proposed description of the critical point in the $S U(2)$ symmetric model? This is simply the $\mathrm{CP}^{1}$ model with a non-compact gauge field and no Berry phase terms in Eq. (1.7). Equivalently it may be thought of as the critical point of the $D=3$ classical $\mathrm{O}(3)$ model when monopoles have been forbidden by hand. This transition was first studied by Kamal and Murthy 39 and more recently by Motrunich and Vishwanath ${ }^{21}$, where it was established that a continuous transition indeed exists that is different from the Heisenberg transition. The non-compact $C P^{1}$ theory Eqn. (1.7) was also directly studied via numerical Monte Carlo methods and found to possess a continuous transition with the same universal properties as the monopole suppressed O(3) NL $\sigma \mathrm{M}$. Numerical results for exponents associated with several observables are available. Further evidence for the continuous nature of the transition in the $\mathrm{CP}^{1}$ model coupled to a noncompact gauge field is obtained by considering the larger class of models with $\mathrm{CP}^{N-1}$ fields coupled to a noncompact gauge theory. It is well known that the $N=1$ model has a continuous transition ${ }^{55}$ which is dual to the XY transition, and a continuous transition is also expected for large values of $N$. Thus, the model of interest $N=2$ is sandwiched between these two extremes where a continuous transition is well known to obtain.

\section{PHYSICAL PROPERTIES NEAR THE 'DECONFINED' CRITICAL POINT}

We now discuss the consequences of the theory for the physical properties near the direct Néel-VBS transition. We will first discuss those properties that follow generally from the (dangerous) irrelevance of monopoles. Later we will specialize to the easy plane limit where the selfduality enables more progress.

It is useful to think first about the various length scales in the problem in the VBS phase.

First there is the spin-spin correlation length $\xi$ which will diverge at the transition. Second, there is a length scale $\xi_{\text {VBS }}$ associated with the 'thickness' of the domain walls of the (discrete) VBS order. The latter is clearly determined by the strength of the quadrupled monopole operator, $\lambda \equiv \lambda_{4}$, in Eq. (1.9); in the easy plane case, $\lambda$ appears as the co-efficient of a local term in the dual action Eq. (5.6). These two length scales will diverge differently - the domain wall thickness will diverge faster than the spin-spin correlation length. One can determine the scaling of $\xi_{\mathrm{VBS}}$ with $\xi$ by a matching argument. On scaling grounds, one expects

$$
\xi_{\mathrm{VBS}} \sim \xi f\left(\lambda \xi^{3-\Delta}\right)
$$

where $f$ is a scaling function, and $3-\Delta$ is the RG eigenvalue of $\lambda$ assuming the scaling dimension of the four monopole operator is $\Delta>3$, and $d=2, z=1$. Beyond the scale of the correlation length $\xi$, one can regard the VBS phase as XY ordered in $\psi_{\text {VBS }}$, though with very weak four-fold anisotropy since $\lambda$ is irrelevant at the QCP. Hence the low-energy variations of the phase $\theta$ of $\psi_{\text {VBS }} \sim\left|\psi_{\text {VBS }}\right| e^{i \theta}$ are described as a pseudo-Goldstone mode, with energy

$$
E(\theta)=\int d^{2} x\left[\frac{\tilde{K}}{2}|\nabla \theta|^{2}-\tilde{\lambda} \cos 4 \theta\right],
$$

where $\tilde{K}$ and $\tilde{\lambda} \propto \lambda$ are renormalized parameters on the scale of $\xi$. A twist of $\theta$ (of e.g. $\pi / 2$ ) is carried hence by a domain wall which, by dimensional analysis, has width $\xi_{\mathrm{VBS}} \sim \sqrt{\tilde{K} / \tilde{\lambda}}$. Knowing then that $\xi_{\mathrm{VBS}} \sim \lambda^{-1 / 2}$, one requires that $f(x) \sim x^{-1 / 2}$ in Eq. (7.1), which implies 33

$$
\xi_{\mathrm{VBS}} \sim \xi^{(\Delta-1) / 2}
$$

Since $(\Delta-1) / 2>1, \xi_{\text {VBS }}$ indeed grows more rapidly than $\xi$ as the QCP is approached.

Thus there are two independent diverging length scales. Either of these length scales may be given several different interpretations. For instance, the spin correlation length $\xi$ may also be interpreted as the length scale at which correlations of the dual global order parameter crossover from that of the critical fixed point to that of 
the (unstable) fixed point which breaks the dual global continuous symmetry. Similarly, the domain wall thickness $\xi_{\text {VBS }}$ of the VBS order is also the length scale at which the photon that couples to the spinons acquires a mass due to instanton effects. This is also the length beyond which the logarithmic Coulomb potential between spinons crosses over to a linear confining one. This is distinct from the 'confinement' length scale describing the spatial size of the resulting two-spinon bound states (triplons). This length scale is actually a non-trivial combination of the two other diverging length scales. It however diverges faster than the spin correlation length $\xi$.

Note that the critical theory is isotropic in space-time and therefore has dynamic scaling exponent $z=1$. The values of other critical exponents may be obtained from the numerical work of Ref. 21. In the $O(3)$ symmetric case the correlation length exponent $\nu \approx 1$ (for $\xi$ ), and the Néel order parameter exponent $\beta \approx 0.80$. Perhaps most remarkably the anamolous dimension of the Néel order parameter field $\eta$ is large $(\approx 0.6)$. This should be contrasted with the extremely small value for $\eta$ at the usual Wilson-Fisher $O(3)$ fixed point in $D=3$ dimensions (and indeed for most other familiar three dimensional critical points). The large value of $\eta$ can be rationalized by the thinking that the Néel order parameter field decays into spinons right at the critical point. Indeed as argued in previous sections it is the spinons which appear as the more natural degrees of freedom at the deconfined critical point. We note however that the spinons are not to be considered 'free particles' - they are critical and furthermore interact through the coupling to the non-compact gauge field.

Consider the effect of twisting the boundary conditions on the VBS order - for instance, for columnar dimerization prefer even columns at one boundary and odd columns at the opposite boundary. Let us suppose the twist is applied between the top and bottom ends ( $y=0, W$ ) of an $L \times W$ sample. On general grounds, the energy cost at long scales will be $E \sim \sigma L^{d-1}=\sigma L$, where $\sigma$ is a "surface tension" or domain wall energy per unit length. This surface tension is set, however, by the irrelevant monopole term and vanishes in a manner set by the divergence of the domain wall thickness. In particular, the surface tension scaling obtains only for twists sustained over a distance $W \gtrsim \xi_{\text {vBS }}$. For twists of the VBS order over a shorter distance $W$ such that $\xi_{\text {VBS }} \gg W \gg \xi$, the energy cost for this twist is greatly reduced to $E \sim K L /(2 W)$, where $K$ is the "stiffness" associated with the continuous dual global symmetry (and we are at length scales where the system has not realized this symmetry is actually discrete). The two energy costs for the twist become comparable for $W \sim \xi_{\mathrm{VBS}}$, so that one expects $\sigma \sim K / \xi_{\mathrm{VBS}}$. This stiffness itself vanishes upon approaching the quantum critical point in a manner set by the divergence of the spin correlation length. Furthermore, the corresponding exponent is the same as for the spin stiffness on the other side of the transition. Specifically, the VBS stiffness $K \sim \xi^{2-d-z}$ where $\xi$ is the dual correlation length, $d=2$ is the spatial dimension, and $z=1$ is the dynamic critical exponent. Thus $K \propto 1 / \xi$.

Note that this is not a test of self-duality but rather a test of the irrelevance of monopoles: the scaling of the VBS stiffness is a consequence of dual current conservation which obtains if monopoles are irrelevant. Thus the same behavior is also expected for the isotropic model.

In practice, a measurement of the domain wall energy in the columnar state is likely best obtained by simply comparing energies of systems of size $L \times W$ (in the $x$ and $y$ directions, respectively) with periodic boundary conditions in both directions (i.e. on the torus) and varying $W$. In particular, let us consider $W>L$, with $L$ odd. In this case, the columns will prefer to align along the short direction (i.e. columns parallel to the $x$ axis, breaking translational symmetry along $y$ ) in order to avoid introducing a domain wall (which would be required in the other orientation due to the odd $L$ ) with energy $\operatorname{cost} \sigma W$. If $W$ is odd, there will still be a domain wall required, but it is shorter and less costly, with energy $\sigma L$. Hence one expects the ground state energy of the system to be $E_{L W} \approx \epsilon W L$ for $W$ even, and $E_{L W} \approx \epsilon W L+\sigma L$ for $W$ odd. Here $\epsilon$ is the ground state energy density. Hence the surface tension can be obtained by

$$
E_{L, W+1}-2 E_{L, W}+E_{L, W-1} \sim 2 \sigma L(-1)^{W}, \quad L \text { odd } .
$$

This behavior will obtain provided $W>L \gg \xi_{\text {VBS }}$. When the system is smaller than the domain wall thickness, however, this energy is determined instead by the stiffness, i.e.

$$
E_{L, W+1}-2 E_{L, W}+E_{L, W-1} \sim K \frac{L}{W}(-1)^{W}, \quad L \text { odd },
$$

for $\xi \ll L<W \ll \xi_{\text {vBS }}$.

It is also clear that at the critical point, both columnar dimer and plaquette order parameters will have power law correlators with the same exponent. This is independent of which one of these two phases we eventually end up in. This is because both order parameters are contained in the dual global boson creation operator. They correspond to the phase of this boson locking in different directions. Which one of these phases is selected is determined by the sign of the anisotropy term, but as this is renormalizing to zero at the critical fixed point, there will be no distinction between the two order parameter correlations at the critical point.

This suggests the following interesting numerical check. Consider, for instance, the situation where the disordered phase has columnar VBS order. Now consider measuring the stiffness to plaquette order in this columnar phase. Since this order is not spontaneous in the VBS phase, measuring this stiffness cannot be accomplished as above by simply comparing systems with odd and even lengths. Instead, one should imagine introducing e.g. two rows separated by half $W$ on which the magnetic couplings have been increased or decreased in a pattern 
mimicking the strong bonds of the plaquette state. The ground state energy of this system should be compared with that obtained by shifting one of these rows by one lattice spacing, and the difference of these two energies interpreted as the energy cost for a twist of the plaquette order parameter. Deep in the phase, this energy cost will be exponentially small, $\Delta E \sim L e^{-W / \xi_{\mathrm{VBS}}}$. However, in the regime $\xi_{\mathrm{VBS}} \gg W \gg \xi$ the cost for the twist of the plaquette order parameter will be determined by the stiffness, i.e. $\Delta E \sim K_{p l} L /(2 W)$. There will thus be a dramatic change from exponential to power-law behavior in this quantity on approaching the critical point.

The coefficient of proportionality $K_{p l}$ will equal the corresponding stiffness $K$ for columnar order and scale identically to the physical spin stiffness on the other side of the transition. Once again, this is not a test of selfduality but follows from the irrelevance of monopoles and will hold in the isotropic case as well.

Specializing to the easy plane case, the self-duality of the critical fixed point implies some further interesting properties. First, it is clear that the dual global boson will have the same power law decay as the physical spincorrelator. The former is identified with the VBS order parameter while the latter corresponds to the staggered $\mathrm{XY}$ correlators in the microscopic spin model. We thus have the remarkable result that the columnar dimer, plaquette, and staggered XY magnetization all decay with the same power law right at the critical point. Furthermore, the $\beta$ exponent for the particular VBS order that actually develops will be the same as the $\beta$ for the spin order. This is because the anisotropy only serves to lock the phase of the dual order parameter. The amplitude is already non-zero in the scaling limit near the critical point.

\section{A. Ordered state}

As elaborated in the previous subsection, there are two diverging length (or equivalently time) scales upon approaching the transition from the VBS side. How does this manifest itself in the Néel ordered side? To understand this first note that in the ordered phase close to the transition there will be 'soft' modes that correspond to the incipient VBS order on the other side of the transition. Indeed the frequency of these modes will go to zero at the critical point. For concreteness consider the case where the VBS order that develops is columnar. Then as is natural there will be a soft mode corresponding to columnar order with frequency vanishing on approaching the critical point. Remarkably there will be other soft modes that correspond to plaquette ordering whose frequency also vanish on approaching the transition. Once again this is despite there being no such order in the VBS phase. This result is already implied by the discussion in the preceding subsection. Indeed both the plaquette and columnar order parameters have power law correlations at the critical point regardless of which one of the two orders actually develops in the VBS phase. Thus it is natural that the frequencies of both modes go to zero on approaching the critical point from the ordered side. Furthermore, both soft mode frequencies vanish in exactly the same universal way on approaching the transition. Formally the columnar and plaquette order parameters are distinguished only by the orientation of the phase of the complex VBS order parameter. In the absence of monopole tunneling events (which tend to pin this phase to certain values), these two distinct order parameter fields will both behave in a common manner determined by the complex VBS order parameter. Thus in the scaling limit near the critical point both the plaquette and columnar order parameters will display the same universal behavior. On general scaling grounds we expect the VBS soft mode frequency $\omega_{\mathrm{VBS}} \sim \rho_{s}$ where $\rho_{s}$ is the ground state spin stiffness of the Néel ordered state.

Despite the common universal behavior of the vanishing frequency of the two distinct VBS soft modes, there will be a small splitting between the two frequencies that is due to the irrelevant (quadrupled) monopole tunneling events. Indeed the information about which of the two VBS orders eventually develops in the paramagnetic phase is contained in this small splitting. If columnar order develops, then the corresponding soft mode will have slightly lower frequency in the ordered phase as compared to the plaquette soft mode. Note that this splitting will go to zero as the critical point is approached as the monopole fugacity renormalizes to zero. This will however vanish in a very different way from the overall VBS energy scale $\omega_{\text {VBS }}$. Indeed this splitting defines a new energy scale that vanishes faster than $\omega_{\text {VBS }}$. Thus we see that two different energy scales also characterize the physics of the ordered phase.

In the easy plane case, one intriguing aspect of our theory is the physics of the vortex cores in the XY ordered phase close to the transition. As discussed extensively, there are two kinds of classical meron vortices which tunnel into each other in the quantum theory. However, the irrelevance of these instanton tunneling events near the transition implies that the Ising order in the core will survive for a very long time.

We have, up to this point, not considered any effects which explicitly break the lattice translational symmetry. Hence the discussion should be read as appropriate for extended, plane wave states of vortices. Crucially, these states may be classified by their (quasi)wavevectors. Consider the continuum theory in which the spatially-oscillating single instanton events are neglected, and only the (irrelevant) quadrupled instanton fugacity $\lambda$ is included. In the dual formulation, the two Ising vortex states appear as relativistic charged particles. They carry a conserved U(1) non-compact gauge charge (physically their vorticity), i.e. the number of these vorticies $N_{1}+N_{2}$ is conserved. There is a discrete $Z_{4}$ global symmetry, which implies that $N_{1}-N_{2}$ is conserved modulo 8 . The latter is a consequence of 
the continuum limit which removes the single instanton events, and is promoted to a continuous $\mathrm{U}(1)$ symmetry (with $N_{1}-N_{2}$ fully conserved) if the quadrupled instantons are also neglected. Physically, then, excitations of the vortex vacuum, i.e. the $\mathrm{XY}$ ordered state, can be labelled by these quantum numbers. And one should expect there to be "quasiparticles" (really "quasivortices" or "quasimerons") carrying an elementary unit $( \pm 1)$ noncompact gauge charge (physically $\pm 2 \pi$ vorticity) and an elementary $Z_{4}$ charge $N_{1}-N_{2}= \pm 1$. These vortices (as befits an XY ordered phase), with a 'core energy' scaling as $1 / \xi$. There will be other gapped excitations (also with a gap of $\mathcal{O}(1 / \xi)$ ) with zero non-compact gauge charge (zero vorticity) and $N_{1}-N_{2}= \pm 2$, which can be viewed as the VBS soft modes, or alternatively as "vortex excitons".

Let us now consider the effects of the neglected oscillating single monopole term. Nä̈vely, this violates the $Z_{4}$ conservation law, and can mix the quasimeron states with $N_{1}-N_{2}= \pm 1$. However, due to the 4-sublattice oscillation, the process in which one meron state is converted to another is accompanied by the addition of a large momentum. In the absence of a sink for this momentum, therefore, even the single instanton term cannot mix the two meron quasiparticle states. At non-zero temperature, thermally excitated excitations with a large gap $(\gg 1 / \xi)$ can provide this momentum, but are present only with an exponentially small probability due to their gap. Hence violations of the conservation of the "Ising" charge of a single quasiparticle are exponentially weak at low temperature. Of course, four quasiparticles with "up" Ising cores can scatter off one another to produce four quasiparticles with "down" Ising cores via the non-oscillating $\lambda$ term. The amplitude for even this process is, however, suppressed by the irrelevance of $\lambda$ if one is near the QCP.

Thus we arrive at the remarkable conclusion that the "elementary" gapped vortex excitations of the XY ordered phase carry a sharp extra Ising quantum number. Ramifications will be explored in Sec. IX A 1

A number of other predictions may also be made on the effects of various perturbations that can be added to the Hamiltonian near the zero temperature critical point.

\section{B. Uniform Zeeman field}

First let us think about the excitation structure in the paramagnetic side. Deep in the phase, the lowest spinful excitations will be $S=1$ which will be gapful. On approaching the transition, due to the diverging 'confinement' length one might naively think that this will break up into spinons. However even with a non-compact gauge field due to the log attraction coming from the photon there will be logarithmic confinement and the lowest energy spin carrying excitations will continue to have $S=1$ This "magnon" is a gauge neutral bound state of two spinons. Now imagine sitting in the paramagnetic side close to the transition, and turn on a Zeeman field along the $z$-axis in spin space. Once the Zeeman energy exceeds the magnon gap, the chemical potential for such magnons becomes positive and they should condense to modify the ground state. This leads to XY antiferromagnetic order. In the non-compact approximation, because this condensate is gauge neutral, it does not create a gap for the photon via the Higgs mechanism. In reality (i.e. beyond the non-compact approximation) what this means is that there will be coexistence between VBS and XY order. As the spinons are not condensed in this phase, there is no disruption of the VBS order (this can be seen e.g. from the fact that the spinons appear as dual "vortices" in the VBS order parameter, while the magnons carry zero dual vorticity).

These considerations hold only provided another transition does not pre-empt magnon condensation as the Zeeman field is increased. This will happen, e.g. if the magnons experience attractive interactions with one another. Indeed, in a Coulomb interacting system, it is natural to expect that the magnons, which are the analog of excitons, will have attractive interactions with one another at long distances, due to the analog of "van der Waals" forces between their fluctuating dipole moments. This attraction, however, competes with other local interactions due to the complex critical physics on scales $\lesssim \xi$, so the outcome is not clear. Therefore, we do not see a clear argument against a continuous magnon condensation transition into a coexistence phase. Likewise, of course, we cannot rule out a direct first order transition. In any case it therefore seems as though a direct second order transition between the magnetically ordered and VBS phases is unlikely at non-zero Zeeman field.

If the coexistence phase exists, it is interesting to contemplate the transition between the coexistence phase and the pure magnetically ordered one (with canted antiferromagnetic order). In the system with XY symmetry, a transition with exactly these symmetries has been studied in Ref. 56. In this paper, it was shown that, despite coexisting superfluid order and the consequent gapless goldstone mode, this transition is in the universality class of a $D=3 \mathrm{XY}$ model, the $Z_{4}$ symmetry breaking perturbation and the coupling to the goldstone mode being irrelevant. Thinking in terms of the dual formulation suggests this analysis should apply here. In particular, both vortex fields $\psi_{1,2}$ remain gapped across the transition, since both phases are XY ordered. Only the composite order parameter $\psi_{\mathrm{VBS}}=\psi_{1}^{\dagger} \psi_{2}$ is involved in the criticality, and it does not couple minimally to the non-compact gauge field. Integrating out the massive vortex fields while keeping a composite $\psi_{\text {VBS }}$ field and the gauge fluctuations describing the Goldstone mode, one arrives at a model equivalent to Ref. 56. Note that this result implies that this critical point is also deconfined in precisely the same sense as the others discussed in this paper. 


\section{Staggered Zeeman field}

Consider the effect of a staggered Zeeman field on the original spin model. First assume easy plane anisotropy in the plane orthogonal to the applied field. The staggered field will always induce some staggered magnetization but we can ask about XY or VBS order superimposed on this. In the $\mathrm{CP}^{1}$ description, a staggered Zeeman field corresponds to a uniform 'magnetic' field that couples to the $z$ component of $\hat{n}$. It is quite clear that there will now be a split transition between the VBS and $\mathrm{XY}$ ordered phases with an intermediate phase with neither XY nor VBS order (but of course with a staggered magnetization).

Consider the approach from the VBS phase. In the presence of the staggered field, one of the two $\mathrm{CP}^{1}$ fields will condense first. This transition is described by the $N=1$ SJ model (and is inverted $D=3 \mathrm{XY}$ ). The resulting phase is the advertised phase with neither XY or $\mathrm{SP}$ order. Actually it is more useful to think of this critical point in the gauge language as a 'deconfined' critical point than just as inverted XY. This is so particularly if one asks about the magnon spectral function at this transition. This will be determined by the spinon dynamics which in turn are coupled to a non-compact $\mathrm{U}(1)$ gauge field. Thus we might again expect anamolously broad spectral functions even though both phases are confined. (Note that total $S^{z}$ is still conserved).

Eventually, as one tunes towards the XY ordered phase, the other $\mathrm{CP}^{1}$ field will also condense leading to $\mathrm{XY}$ order. This transition is actually exactly dual to the other one discussed above. This is because the staggered Zeeman field couples to the same operator in both the $\mathrm{CP}^{1}$ and dual representations. For small staggered Zeeman fields, the phase boundaries must come in with the same exponents, etc.

Finally, in the $\mathrm{O}(3)$ model, the second transition will not happen as the Néel vector will immediately line up with the staggered field. However, the first transition will continue to be described by the $N=1 \mathrm{SJ}$ model. Details of the slopes of various phase boundaries etc. may be found Ref. 21 - in the terminology of that reference, a uniform field corresponds to the staggered Zeeman field discussed here.

\section{Finite temperature transitions}

Finite temperature properties near the transition may also be discussed. Here it is clearly necessary to distinguish between the easy plane and isotropic cases. In the latter, the Néel order does not survive for any non-zero $T$ while in the former case there is power law order at low $T$ which eventually disappears through a BerezinskiKosterlitz-Thouless (BKT) transition. In both cases however the discrete broken lattice symmetry of the VBS phase will survive upto a non-zero finite $T$. The associated finite- $T$ transition will be in the universality class of the $Z_{4}$ clock model in $d=2$. This transition is known to be described by a line of fixed points with continuously variable (i.e. nonuniversal) critical exponents. The line of fixed points results from the exact marginality of the fourfold symmetry breaking term $\psi_{\mathrm{VBS}}^{4}$, i.e. our $\lambda$ coefficient. As $\lambda$ approaches zero, the nature of the criticality approaches that of a simple $D=2$ classical XY model, i.e it becomes BKT-like. Thus $\eta \rightarrow 1 / 4$ and $\nu \sim 1 /|\lambda|$ diverges in this limit. Since at the zero temperature QCP instantons are irrelevant, we may conclude that the fixed point value of $\lambda$ at the classical VBS-paramagnet transition (which is generally finite and non-zero at $T_{c}$ ) approaches zero as $T_{c} \rightarrow 0$. Hence the non-universal critical behavior of the VBS-paramagnet transition becomes arbitrarily close to BKT behavior as this transition line is followed into the $T=0$ QCP. This conclusion is independent of the $\mathrm{XY}$ or $\mathrm{O}(3)$ symmetry of the magnetic ordering.

In the XY case, the self-duality of the easy plane fixed point implies further that the phase boundaries associated with the finite- $T$ transition from both the Néel and VBS phases have the same shape at low $T$. Note that both are BKT-like for $T_{c}$ asymptotically close to zero (i.e. near the QCP), consistent with duality. Indeed, one expects not only the phase boundary but also all critical correlations to match in this limit, including amplitude ratios.

\section{DECONFINED QUANTUM CRITICALITY AT THE VBS TO SPIN LIQUID TRANSITION}

In this section we argue that the transition between a valence bond solid and a fractionalized spin liquid is also an example of a deconfined quantum critical point in a precise sense.

In two spatial dimensions, a fractionalized spin liquid is expected to be described as the deconfined phase of a $Z_{2}$ gauge theory with a gapped $Z_{2}$ vortex - the vison. This $Z_{2}$ gauge field is minimally coupled to spin- $1 / 2$ spinon excitations. We only consider the case where the spinons are gapped. A precise theoretical characterization is given by the notion of topological order ${ }^{16.57}$.

Consider the evolution of the ground state of a spin$1 / 2$ system (or equivalently for bosons at half-filling) between such a fractionalized spin liqiud and a VBS on, say, a square lattice. Despite the lack of any obvious local order parameter for the spin liquid there is a close similarity with the Néel-VBS transition. Indeed both the spin liquid and VBS are characterized by two distinct types of order (the former by topological order and the latter by broken lattice symmetry). Nä̈ve thinking might then suggest that a direct second order transition is not possible. Rather one might have expected two transitions with an intermediate "coexistence region" which breaks lattice symmetry but is also topologically ordered (a VBS* phase, in the notation of Ref. 17). Once again this nä̈ve expectation is incorrect and a direct second order tran- 
sition is indeed possible. Furthermore the critical theory may be regarded as a non-compact $\mathrm{U}(1)$ gauge theory with an extra emergent dual global U(1) symmetry.

It is convenient to begin with a theoretical formulation that is powerful enough to describe both phases and all of their distinct excitations. Such a formulation is provided in the work of Refs. 1516 58. As before, the underlying spin model is first reformulated as a theory of spin- $1 / 2$ spinon fields that are minimally coupled to a compact U(1) gauge field with Berry phases. The VBS corresponds to a confined paramagnet where the spinons have disappeared from the spectrum. The spin liquid obtains when a singlet pair of spinons - which carries gauge charge-2 - condenses i.e enters a Higgs phase. Let us represent this Higgs field by the operator $Q=e^{i \varphi}$. We imagine integrating out the individual spinon fields (this is permissible because all spin carrying excitations are gapped in both the VBS and spin liquid phases), and obtain the following theory for the transition ${ }^{17.51}$

$$
\begin{aligned}
\mathcal{S} & =\mathcal{S}_{\varphi}+\mathcal{S}_{a}+\mathcal{S}_{B} \\
\mathcal{S}_{\varphi} & =-2 t \sum_{\ell} \cos (\boldsymbol{\Delta} \varphi-2 \mathbf{a})
\end{aligned}
$$

where $\mathcal{S}_{a}$ and $\mathcal{S}_{B}$ are defined in Eqs. (3.12) and (3.13). Note that Eq. 8.1) is just the $N=1$ SJ model studied in Section IVA but with the crucial difference that $\varphi$ carries charge 2 (compare with Eq. (4.1)). The duality transformations of Section IVA are easily applied to Eq. (8.1), and we obtain an XY model with 8-fold anisotropy which is irrelevant at the transition (this contrasts with the 4fold anisotropy obtained in Section IVA.

In the spirit of previous Sections (particularly Section IVA), these results may be understood physically as follows. In the fractionalized phase the condensation of the charge- 2 scalar leads to vortex excitations (the visons) which carry $\pi$ gauge flux. In the fractionalized phase instanton effects kill visons in pairs - indeed this is precisely what leads to their 'Ising' nature. The transition to the confined VBS phase occurs when the visons condense. But near the transition, and in the continuum limit, we expect once again that all instanton events are quadrupled. Thus the $\pi$ flux vortices can only disappear 8 at a time. This gives the XY model with 8 -fold anisotropy.

We thus see that the 8-fold anisotropy in the dual $\mathrm{XY}$ model should be interpreted as instanton tunneling events in the original compact gauge theory. Consequently as before we conclude that instantons are irrelevant at the critical fixed point so that a gapless noncompact $\mathrm{U}(1)$ gauge theory obtains. (We remind the reader that the global XY model is the dual of the condensing charge-2 scalar coupled to a non-compact gauge field).

Note once again the crucial role played by the Berry phases which are responsible for leading to an 8-fold anisotropy (as opposed to 2-fold as would obtain in their absence).
Note also that spinons are well-defined in the fractionalized phase but are confined in the VBS phase. What is the fate of the gapped spinons right at the transition between these two phases? The arguments above show that at the critical point the spinons are minimally coupled to a non-compact $\mathrm{U}(1)$ gauge field descending from $a_{\mu}$ (which in turn is also coupled to the critical spinon pair field). The strong scaling properties of this critical point (which is dual to the $D=3 \mathrm{XY}$ model) implies that the gauge-field has the following two-point correlator at criticality

$$
\left\langle a_{\mu}(p) a_{\nu}(-p)\right\rangle \sim \frac{1}{p}\left(\delta_{\mu \nu}-\frac{p_{\mu} p_{\nu}}{p^{2}}\right),
$$

where $p_{\mu}$ is the spacetime 3 -momentum. Note that this propagator does not have the Maxwell $1 / p^{2}$ scaling, but a $1 / p$ dependence fixed by the scaling dimension $\operatorname{dim}\left[a_{\mu}\right]=$ 1. This implies a $1 / r$ interaction between static massive spinons at criticality.

It is sometimes stated that the transition between the VBS and spin liquid phases is described by a $Z_{2}$ gauge theory. The results here however show that the transition is in fact described as a deconfined $\mathrm{U}(1)$ gauge theory in a very precise sense. It is the spin liquid phase itself (as opposed to the transition) that is described as a (deconfined) $Z_{2}$ gauge theory.

\section{A. Spin liquids that break lattice symmetry}

An important subtlety has been glossed over in the analysis so far in this section. Spin liquid states with no broken lattice symmetries are certainly possible 16.17.59.60, and for these the above analysis applies. However, in bosonic mean field theories of $\mathrm{SU}(2)$ spin liquid states on a variety of lattices 58.61 .62 .63 .64 , the spin liquid state is commonly found to break a global lattice rotation symmetry ${ }^{65}$ - such a state has 'bond-nematic' order. The spin liquid is associated with short-range, incommensurate spin correlations at a wavevector $K$, and the choice of $K$ often breaks a lattice symmetry e.g. a spin liquid state at $K=(k, k)$ is distinct and inequivalent to a state at $K=(k,-k)$. Such states appear naturally at the boundary of a VBS state ${ }^{58}$, and for these the theory above has to be reconsidered. Before doing this, we note one important case for which this is not necessary: the $\mathrm{Cs}_{2} \mathrm{CuCl}_{4}$ lattice ${ }^{61.63}$, which interpolates between the square and triangular lattices. Within a large $N$ bosonic mean field theory treatment, the ground state in the square lattice limit is a VBS, while in the triangular limit it is spin liquid which breaks no lattice symmetries: the transition between these states is described by the theory in Eq. 8.1).

Turning to a spin liquid that does break lattice symmetries, consider e.g. the transition on the square lattice ${ }^{58}$ from the VBS (Fig 1) with short-ranged spin correlations peaked at the wavevector $(\pi, \pi)$, to a bond-nematic spin liquid at wavevector $K=(k, k)$ or $K=(k,-k)$. 
The choice of either of the latter states breaks a symmetry of reflection about the principal square lattice axes. In mean field theory, ${ }^{58}$ this transition is characterized by the condensation of two Higgs fields, which we denote as $Q_{x}=e^{i \varphi_{x}}$ and $Q_{y}=e^{i \varphi_{y}}$. These fields are odd under the lattice reflections $\mathcal{R}_{x}$ and $\mathcal{R}_{y}$ in Table I respectively, ${ }^{65}$, and this prohibits terms which are linear in either Higgs field in the effective action. Using these symmetries, and the requirements of gauge invariance, we generalize Eq. 8.1) to

$$
\begin{aligned}
& \mathcal{S}=\mathcal{S}_{\varphi}+\mathcal{S}_{a}+\mathcal{S}_{B} \\
& \mathcal{S}_{\varphi}=-\sum_{\ell}\left[2 t \cos \left(\boldsymbol{\Delta} \varphi_{x}-2 \mathbf{a}\right)\right. \\
& \left.\quad+2 t \cos \left(\boldsymbol{\Delta} \varphi_{y}-2 \mathbf{a}\right)+2 t^{\prime} \cos \left(2 \varphi_{x}-2 \varphi_{y}\right)\right]
\end{aligned}
$$

where, as before, $\mathcal{S}_{a}$ and $\mathcal{S}_{B}$ are defined in Eqs. (3.12) and (3.13). Note the crucial factor of 2 in the argument of the third cosine in Eq. (8.3): this is required by the inversion constraints above. Apart from the usual compact U(1) gauge invariance, Eq. (8.3) is also invariant under the global $Z_{2}$ transformation

$$
\begin{aligned}
& \varphi_{x} \rightarrow \varphi_{x}+\pi / 2 \\
& \varphi_{y} \rightarrow \varphi_{y}-\pi / 2
\end{aligned}
$$

which realizes the lattice reflection symmetry. (Note that the square of the transformation in Eq. (8.4) is equivalent to the identity modulo a compact $U(1)$ gauge transformation.) Consequently, there are now two inequivalent Higgs phases, with $\left\langle\varphi_{x}-\varphi_{y}\right\rangle=0$ (or $\left.\left\langle Q_{x}\right\rangle=\left\langle Q_{y}\right\rangle\right)$ and $\left\langle\varphi_{x}-\varphi_{y}\right\rangle=\pi\left(\right.$ or $\left.\left\langle Q_{x}\right\rangle=-\left\langle Q_{y}\right\rangle\right)$, and these correspond ${ }^{58}$ to the two possible spin liquid phases at $K=(k, k)$ and $K=(k,-k)$. The theory Eq. (8.3) can be analyzed by the same duality transformation applied to Eq. (8.1), but the critical properties have not been determined.

\section{ANALOGIES AND EXTENSIONS}

\section{A. Superfluid-insulator transition of correlated bosons}

The models and the phenomena discussed in this paper can be fruitfully discussed from a different point of view. Consider a system of bosons with short-ranged repulsive interactions on a square lattice such that there is half a boson per site on average. It has long been appreciated that such a bosonic system is closely related to quantum spin models with easy plane (or easy axis) anisotropy. Indeed, there is an exact equivalence in the hard-core limit in which at most one boson occupies each lattice site. Specifically, one may consider a model of bosons (described as $\mathrm{O}(2)$ quantum rotors) on a square lattice:

$$
H=U \sum_{r}\left(n_{r}-\frac{1}{2}\right)^{2}-t \sum_{\left\langle r r^{\prime}\right\rangle} \cos \left(\phi_{r}-\phi_{r}^{\prime}\right)+\ldots
$$

Here $\phi_{r} \in[0,2 \pi)$ represents the boson phase, $n_{r}$ is the conjugate boson number and is an integer $\in[-\infty, \infty]$. The ellipses represent other short ranged terms that can be tuned to drive transitions from a superfluid to (for instance) the bond stripe insulator. To relate the above boson Hamiltonian to the antiferromagnetic systems considered in the bulk of the paper, we note that one may define

$$
\begin{aligned}
S_{r}^{ \pm} & =\epsilon_{r} e^{\mp i \phi_{r}}, \\
S_{r}^{z} & =n_{r}-\frac{1}{2} .
\end{aligned}
$$

For large $U$ this gives a faithful representation of an easyplane spin- $1 / 2$ antiferromagnet, and the universal physics is expected to be unchanged at smaller $U$.

Clearly a superfluid phase of the bosons is possible (and corresponds to the XY ordered phase in the magnet analogy). Various kinds of Mott insulating ground states are also possible. (These correspond to quantum paramagnets in the magnetic case). A simple Mott state corresponds to the bosons forming a checkerboard ordered pattern in which the sites of one sublattice are preferentially occupied. This will be stabilized by large nearest neighbor repulsion and corresponds to the Ising ordered antiferromagnet. In the boson language the columnar VBS state may be understood as a 'bond-centered' stripe (or a bond density wave) - a state in which each boson is shared in a bond between two nearest neighbor sites such that these favored bonds have lined up in columns. The considerations of Section $\nabla$ more or less apply directly to the transition between the superfluid and the bond-centered stripe insulator (or the analogous plaquette ordered insulator). In particular the critical theory is 'deconfined' and is expressed in terms of two fields each with boson charge- $1 / 2$ that are minimally coupled to a non-compact U(1) gauge field. However the discussion in Section $\nabla$ was intended for weak easy plane anisotropy on an isotropic spin model. It is somewhat more satisfying to derive the crucial field theory Eq. (5.6) directly for the bosonic system. We point out that the approach of Ref. 48 provides such a direct derivation of the required dual action. However the close connection with fractionalized charge degrees of freedom is somewhat obscured by that approach. We therefore sketch in Appendix E a derivation proceeding in a manner more similar to the considerations of the previous sections, in particular going directly from the boson model of Eq. (9.1) to the dual meron action obtained earlier.

In the context of boson models (in view of potential applications e.g. to atomic bosons in optical lattices or to electronic systems where the bosons are Cooper pairs), some physical properties arise which are less natural in the context of quantum antiferromagnets discussed earlier. In particular, it is interesting to consider the effects of an applied orbital magnetic field coupling. This can bring out the unusual physics of Ising ordering in the vortex cores discussed earlier in Sec. VIIA 


\section{Orbital magnetic field}

Let us consider the structure in an applied orbital magnetic field $B$. The QCP at zero field describes a transition between a superfluid phase and a bond-centered striped phase.

Suppose the system is on the superfluid (XY ordered) side of the QCP, and a small magnetic field is applied (we use the internal field $B$ ). This field produces vortices, separated by an average distance $\ell=\sqrt{\phi_{0} / B}$, where $\phi_{0}=h c / q$ is the flux quantum, and $q$ is the boson charge. In the weak field limit, where the length $\ell$ is large, one expects these vortices to form an Abrikosov lattice, since the long-range logarithmic interactions between vortices dominate their kinetic energy. Now suppose one is near the QCP, so that the correlation length $\xi$ is large. To a first approximation, one can neglect instanton events, and treat the Ising quantum number of the vortices as conserved. Then each vortex in the Abrikosov lattice has a definite Ising "charge", and hence the system as a whole some sort of Ising magnetic order. It is straightforward to see that the basic interactions between these Ising "spins" are antiferromagnetic, and that these interactions decay rapidly when the two vortices in question are separated by a distance much larger than $\xi$. These interactions arise because the two types of vortices carry opposite gauge flux $\int d^{2} x \epsilon_{i j} \partial_{i} a_{j}= \pm \pi$. This gauge flux is confined to a region of the size of the (gauge) "penetration depth". Since the gauge field fluctuations are part of the quantum critical theory, this penetration depth is of the $\mathcal{O}(\xi)$. Due to the Maxwell term in the action, two nearby vortices have lowest energy with opposite gauge fluxes (and hence smaller total gauge flux), provided the two fluxes overlap. A mean field analysis following Abrikosov leads to the same conclusion as discussed below. In particular, consider the Lagrangian $\mathcal{L}\left(z_{\alpha}\right)$ (in Eqn. 1.7) for the $z_{\alpha}$ fields. We are interested in $w<0$ (in Eqn. [1.8), and it is convenient to consider the limit $w=-2 u+\delta w$, with $0<\delta w \ll 2 u$. For $\delta w=0$, the mean field theory (which neglects fluctuations of $\vec{a}$ ) comprises simply of two decoupled copies of Abrikosov's lattices for $z_{1}$ and $z_{2}$. Thus the solution consists of a triangular vortex lattice in each $z_{\alpha}$, with lattice spacing $\sqrt{2} \ell$ (since each $z_{\alpha}$ has charge $q / 2$ ). These two lattices are completely decoupled in this approximation. With $\delta w>0$, the energy is minimized when the integral of $\left|z_{1}\right|^{2}\left|z_{2}\right|^{2}$ is smallest. This is accomplished by placing the $z_{1}$ and $z_{2}$ vortices as far apart as possible, so that $\left|z_{1}\right|^{2}\left|z_{2}\right|^{2}$ is reduced over the maximum spatial area. The solution is to choose the two triangular $z_{\alpha}$ vortex lattices as the two sublattices of a honeycomb lattice. This corresponds to an antiferromagnetic orientation of the Ising vortex cores on the honeycomb.

To establish the stability of this order, we must reconsider the effect of instanton events in this phase. The important events are single instantons, which act like a transverse field on the Ising quantum number. While these average away in the continuum theory, the finite lattice spacing $\sim \ell$ provides an upper length cutoff for the oscillations of the single instanton fugacity, which can therefore have an effect. Near the QCP, it is possible for both $\ell$ and $\xi$ to be large, but to have $\ell$ not much greater than $\xi$. In this limit, which we consider, the overlap of the vortex cores is strong, hence the Ising antiferromagnetic "exchange energy" between neighboring vortices is large, i.e. of order $1 / \xi$ by scaling. The effective transverse field on the vortices is more difficult to estimate. In a mean field treatment, one simply averages the oscillating instanton fugacity over the two dimensional $\sim \xi^{2}$ using some smooth envelope function. This gives a transverse field $\sim \lambda_{0} / \xi^{2}$. Fluctuation effects may be expected to further decrease this field. Hence, the transverse field is much weaker for large $\xi$ than the antiferromagnetic coupling between cores.

Thus we arrive at the remarkable conclusion that the vortex state near the QCP exhibits antiferromagnetic Ising LRO of the staggered Ising magnetization of the vortex cores. Note that this analysis applies when the magnetic length $\ell \sim \xi$. For very small fields, or further from the QCP, $\ell \gg \xi$, and the antiferromagnetic interactions between cores $\left(\sim e^{-\ell / \xi}\right)$ decay exponentially, while the transverse field is likely of power law form. Hence for very small fields it seems probable that the Ising cores become disordered. In this case, the physical manifestation of the long-lived Ising staggered magnetization is the presence of a low energy "antibonding" excitation of each vortex.

Clearly, upon increasing the quantum fluctuations, this vortex lattice must disappear, since the VBS state on the other side of the critical point is an orbital paramagnet, i.e. no change in symmetry occurs on applying a weak field to it. Hence there is at least one phase boundary separating the Ising ordered vortex lattice from the VBS phase that persists at $B>0$. We will not address this "vortex lattice melting" physics here, except to say that first order, continuous, and multi-stage transitions (with intermediate partially ordered phases) are all possible in principle (and difficult to distinguish between on purely theoretical grounds).

\section{B. Higher spin}

In the bulk of this paper we have focused on the spin-1/2 square lattice antiferromagnetic model. Here we briefly discuss the fate of higher spin models on the square lattice. It should be clear by now that the answers depend crucially on the Haldane phases that obtain for higher spin. In the isotropic model, if $2 S=1(\bmod 4)$ then the monopoles are quadrupled. Thus for all such values of the spin, a direct second order Néel-VBS transition described by the same deconfined critical theory as for spin- $1 / 2$ obtains. If $2 S=0(\bmod 4)$, then there are no oscillating phase factors for the monopoles. This has the consequence that a translation symmetric quantum paramagnetic state is now possible. The transition 
to this state from the Néel state will be described by the usual LGW $\mathrm{O}(3)$ fixed point (i.e. with monopoles present). If $2 S=2(\bmod 4)$ then the appropriate Haldane phases lead to doubling of monopole events. Now confined paramagnetic states necessarily break lattice symmetries. Whether a direct second order Néel-VBS transition is allowed or not depends on the scaling dimension of the 2-monopole operator at the monopole-suppressed fixed point. If this is irrelevant, then the same deconfined critical theory as for spin- $1 / 2$ will obtain.

It is interesting to consider the spin- 1 case in the presence of some easy plane anisotropy. This may equivalently be viewed as a model of bosons at integer filling unlike in the isotropic limit, a translation symmetric confined paramagnet is clearly possible. A direct transition between the XY ordered phase and such a paramagnet is clearly possible and will be in the usual $D=3 \mathrm{XY}$ universality class.

However, presumably the interesting question even in the easy plane case is whether a direct second order transition is possible between the $\mathrm{XY}$ ordered phase and a lattice symmetry broken confined paramagnet with bond order. To answer this question and to obtain a description of such a paramagnet, it is convenient to start from the isotropic limit and introduce weak easy plane anisotropy. In the isotropic limit the confined paramagnetic states will break lattice symmetries and this will be preserved upon turning on some easy plane anisotropy. In a $\mathrm{CP}^{1}$ description, there will now be monopole Berry phases that oscillate on two sublattices of the dual lattice. We may now dualize in the easy plane limit to the meron vortex degrees of freedom. In this description it is clear that the translation broken VBS state is again described by an equal amplitude condensate of both vortex fields. Now the instanton term converts two merons of one kind into two of the other kind i.e. the co-efficient of the $\lambda$ term in Eq. (5.6) is $\operatorname{Re}\left[\left(\psi_{1}^{*} \psi_{2}\right)^{2}\right]$, with $\lambda \equiv \lambda_{2}$. The relevance/irrelevance of this at the self-dual, easy plane, non-compact $\mathrm{CP}^{1}$ fixed point will determine whether a direct second order transition obtains or not: this question remains open at present.

Note that near the transition to the usual paramagnet with no broken symmetries there will only be single species of vortex with a featureless 'paramagnetic' core. On the other hand near the transition to the VBS phase there will once again be two species of (nearly) stable vortices with cores that have very long-lived Ising order (if as discussed above such a direct continuous transition is possible).

\section{Honeycomb lattice}

The considerations of this paper generalize readily to other two dimensional bipartite lattices. For instance on the honeycomb lattice the Haldane Berry phase calculation implies that all monopole events are tripled (rather than quadrupled as on the square lattice). This implies that the issue of whether or not a direct second order transition described by a 'deconfined' critical point obtains between the Néel and VBS phases is determined by the scaling dimension of the 3-monopole operator at the monopole-suppressed fixed point. Unlike the square lattice it is however less clear that the 3-monopole operator will be irrelevant. For instance for the $N=1 \mathrm{SJ}$ model, the Higgs-VBS transition is determined by the $Z_{3}$ clock universality class which is distinct from the XY universality class. In other words the 3 -fold anisotropy which represents instantons is relevant at the deconfined fixed point at $N=1$. At large- $N$ all monopoles continue to be irrelevant. The fate of the physical models (with and without easy plane anisotropy) can only be settled by direct numerical computation of the scaling dimension of the 3-monopole operator.

\section{Ising Anisotropy and Other Transitions}

In this paper, we have focused on the properties of spin-1/2 antiferromagnets with full $\mathrm{SU}(2)$ spin rotational symmetry, or its easy-plane reduction to $\mathrm{U}(1)$. These two cases are amenable to analysis due, on the one hand, to natural continuations of the $\mathrm{SU}(2)$ invariant $\mathrm{CP}^{1}$ representation to $\mathrm{CP}^{N-1}$, and through standard $\mathrm{XY}$ duality. One may also ask whether similar deconfined critical points might arise in systems with easy-axis (i.e. Ising) anisotropy, which also retain the $\mathrm{U}(1)$ subgroup of $\mathrm{SU}(2)$. Unfortunately, this limit is much less amenable to microscopic duality transformations on the lattice level, and so it is difficult to make firm statements. While some of us suspect that no deconfined critical behavior is likely in this case, it is nevertheless of interest to present candidate field theories for such deconfined transitions.

Very naively, one may attempt to begin with the $\mathrm{CP}^{1}$ representation of the quantum antiferromagnet, and simply change the sign of the anisotropy term, taking $w>0$ in Eq. (1.8). In a mean-field analysis of the continuum field theory of Eq. (1.7), including anisotropy of this sign would indeed have the desired effect of yielding a transition between an Ising ordered phase (for $s<0$ ) and a VBS phase (for $s>0$ - actually one naïvely obtains the Coulomb phase of the gauge theory, neglecting the dangerously irrelevant instantons). There are, however, several caveats to this candidate theory that must be mentioned. First, supposing the gauge field $a_{\mu}$ non-compact, fluctuation effects are known in some situations (e.g. the classical Abrikosov transition between the normal state and vortex lattice at $H_{c 2}$ ) to drive nä̈vely continuous transitions involving gauge fields first order. While we believe this does not occur in the cases of $\mathrm{SU}(2)$ invariance and $\mathrm{XY}$ anisotropy, these conclusions are based on several exact dualities and the numerical results of Ref. 2139 directly on models in which instantons have been suppressed, and direct simulations of $\mathrm{CP}^{1}$ models coupled to non compact gauge fields ${ }^{21}$. Second, even if the non-compact transition is continuous, to constitute a 
stable deconfined QCP, it must be stable to the (quadrupled) instanton events allowed by the microscopic compact model.

At present we do not have supporting evidence in favor of either of these two conditions. It would be of some interest to develop a semiclassical description of the above scenario to better evaluate it in physically intuitive terms. We note that XY anisotropy, for very simple reasons, favors a "deconfined" critical scenario. In particular, weak XY anisotropy converts the topological defects (solitons) of the antiferromagnet from skyrmions to merons, "fractionalizing" them already in the antiferromagnetic phase. Adding Ising anisotropy instead renders the topological defects local "droplets", or domains of antiphase ordering. These can apparently be viewed as distorted skyrmions, in which the smooth rotation from the anti-aligned core to infinity is replaced by a domain wall of finite width. Thus there is no fractionalization of the topological defects in the Ising antiferromagnet, although they do appear to carry the integer skyrmion quantum number.

Nevertheless, the action in Eqs. (1.7) and (1.8) appears to describe a putative deconfined Ising AF to VBS transition. There is clearly no self-dual description of this QCP, since neglecting instantons, the VBS phase is replaced by a Coulomb phase with a gapless photon, while the Ising AF has no gapless excitations. Formally, however, one may wonder what physics might be represented by considering the mathematically similar "anisotropy" in the dual meron theory, i.e. taking $w_{d}>0$ in Eq. (5.6). Provided this transition remains continuous in $2+1$ dimensions and the $\lambda$ term remains irrelevant in this case, this would describe a different quantum phase transition. In particular, for $s_{d}>0$, the ground state has no vortices and there is a Meissner response (Maxwell term for $A_{\mu}$ ), hence it describes an XY superfluid. For $s_{d}<0$, with $w>0$, one or the other (not both) types of merons condense and the $A_{\mu}$ gauge field develops a Higgs mass. Hence this describes a non-superfluid state. From Table III one sees that the non-zero expectation value of $\left|\psi_{1}\right|^{2}-\left|\psi_{2}\right|^{2}$ implies Ising AF order. Thus this critical point describes a putative direct continuous transition between XY and Ising antiferromagnets.

Thus these two theories describe different potential routes of "disordering" a quantum Ising antiferromagnet with $\mathrm{U}(1)$ spin-rotation symmetry, either to a VBS phase or an XY antiferromagnetic phase. If they are indeed continuous, with the instanton fugacity and $\lambda$ term respectively irrelevant, they are not self-dual but instead dual to one another. It would be interesting to determine with more certainty whether these putative critical theories can survive fluctuation effects. We note that the numerical simulations of Ref. 5 observed a first order transition between VBS and Ising antiferromagnetic phases. While this does not rule out the possibility of a continuous transition in other microscopic models, it is perhaps some evidence to the contrary.

\section{EXPERIMENTS}

We now briefly discuss the implications of the phenomena discussed in this paper for experiments on quantum magnets. What are some good signatures of these phenomena in experiments? Imagine a quasi two dimensional Mott insulator where each layer has a square lattice of localized spin-1/2 moments. Ignoring all effects due to coupling between the layers and to other degrees of freedom (phonons,etc), a direct second order zero temperature transition between the Néel ordered and translation broken VBS phases should be possible (for instance by application of pressure) with properties described as in previous sections. It is first important to emphasize that the proposal of a deconfined critical point is on firmest ground for a system with a spin- $1 / 2$ moment per unit cell. With higher spin or with more than one spin-1/2 moment per unit cell other (more ordinary) kinds of phase transitions may well obtain. In practice (even with spin-1/2 per unit cell) the growing VBS fluctuations associated with the lattice symmetry breaking will couple strongly to lattice disortions particularly at low temperature. If the phonons can be regarded as three dimensional (even though the magnetic interactions may be well approximated as two dimensional), a small region of coexistence will most likely be introduced at very low temperature. This may be roughly understood as follows. The elastic energy cost of a latttice disortion of magnitude $x$ that couples to the VBS order parameter is of order $x^{2}$. However the electronic energy gain is much bigger (as the susceptibility associated with the VBS order parameter diverges at the transition), going as $x^{\kappa}$ with $\kappa<2$. In the easy plane case $\kappa \approx 1.35$ from the numerical results 21 . Thus the phonons will then prempt a direct Néel-VBS transition and introduce a small coexistence region. It will thus be necessary to look at temperatures that are not too low to meaningfully compare with experiments.

Barring these caveats the interesting critical phenomena discussed in this paper should be visible in a number of different experimental probes. Scaling forms for various experimentally physical observables are readily written down. For instance, right at the critical point the spin response function $\chi\left(k_{i}, \omega\right)$ near the ordering wavevector $\left(Q_{i}=(\pi, \pi)\right)$ will take the form

$$
\chi\left(k_{i}, \omega\right) \sim \frac{1}{k^{2-\eta}} F\left(\frac{\omega}{c k}, \frac{\hbar \omega}{k_{B} T}\right)
$$

Here $k_{i}$ is assumed to measure the deviation of the physical wavevector from $Q_{i}$, and $T$ is the temperature. The corresponding spectral function can be directly measured in neutron scattering experiments. At a fixed small wavevector $k_{i}$ and temperature $T$, this will show sharp spin wave peaks as a function of frequency in the Néel state and similar sharp 'triplon' peaks near the spin gap in the VBS state. However right at the critical point, there will be an anamolously broad power law peak (due to the large $\eta$ ). 
The large value of $\eta$ will also directly manifest itself in NMR experiments. Indeed the nuclear spin lattice relaxation rate is essentially given by

$$
\frac{1}{T_{1}} \sim T \int d^{2} k \lim _{\omega \rightarrow 0} \frac{\chi^{\prime \prime}(\vec{k}, \omega)}{\omega}
$$

where $\chi^{\prime \prime}$ is the imaginary part of the spin response function. It is now easy to see from scaling that $1 / T_{1} \sim T^{\eta}$ at finite temperatures in the "quantum critical" region. Thus this experiment provides a direct measurement of $\eta$. It is therefore an excellent way to experimentally distinguish the predictions of the present paper from those of the earlier accepted theory ${ }^{33}$ of the Néel-VBS transition which gives $\eta \approx 0$.

In this context it is interesting to reconsider experiments measuring the spin- lattice relaxation rate of the $C u$ ions in the undoped and lightly doped cuprates 66 . Remarkably at high temperature the $1 / T_{1}$ saturates to a temperature and doping independent value. One suggested explanation ${ }^{33}$ is that at these high temperatures in the undoped sample the system is in a quantum critical regime associated with a disordering transition of the Néel order such that $\eta \approx 0$. Furthermore the effects of doping has been suggested to only make the system appear closer to the critical point (at least for the high temperature spin physics). The results of the present paper imply that if this interpretation of the experiments in terms of proximity to quantum criticality is correct, then the corresponding transition cannot be from the Néel to the VBS state.

\section{DISCUSSION}

This paper has described a variety of quantum critical points in two dimensions which can be understood using the new paradigm of 'deconfined' quantum criticality $\stackrel{9}{\text {. }}$. The critical point has an emergent topological conservation law, and the critical theory is expressed most naturally in terms of fractionalized degrees of freedom. The order parameters characterizing the phases flanking the critical point emerge at large length scales as composites of the fractionalized modes, or their duals. These examples clearly violate the LGW paradigm, in that the order parameters are not directly related to the critical modes.

Our primary example was the Néel to VBS transition for the $S=1 / 2$ square lattice antiferromagnet. We showed that a deconfined critical point scenario emerged in a number of tractable deformations of models appropriate to describe this transition. Several results existing in the literaure (for instance on large- $N$ models) were shown to support this proposal when correctly interpreted. We briefly reiterate a few key physical aspects of this theory. First, the critical theory possesses an extra global topoogical conservation law (associated with skyrmion number). It is most naturally expressed not in terms of the natural order parameters of either phase but in terms of new spin- $1 / 2$ spinon degrees of freedom that are specific to the critical point. The emergence of these fractional spin fields at the critical fixed point manifests itself quantitatively in the large value of the anamoulous exponent $\eta$ at the transition. The extra topological conservation law is obtained because monopole events at which skyrmion number can change are irrelevant and disappear at long scales at the critical fixed point. However they are relevant in the paramagnetic phase and lead to the appearnce of broken lattice symmetry. There are two diverging length or time scales near the critical point. In the paramagnetic phase the first (shorter) length is the spin correlation length. There is a longer length scale at which the VBS order gets pinned.

We also considered a number of other critical points in this paper. The transition between VBS and spin liquid states was discussed in Section VIII and described by critical theory closely related to that for the SJ models. The superfluid-insulator transition of bosons at half-filling on the square lattice was considered in Section IX A the insulator exhibits bond-density-wave order and the theory for the critical point (Eq. (5.6)) had been obtained earlier ${ }^{48}$. Here we provided a physical reinterpretation of this theory, and showed how it could also be understood as a deconfined QCP.

Overall our work shows that such deconfined quantum criticality is quite common in two dimensional systems with a spin-1/2 moment per unit cell. This leads us to suspect that the scope for finding deconfined QCP's in other correlated electron systems, including those with fermionic excitations, is bright. These QCP's naturally have large anomalous dimensions for observable order parameters ${ }^{8}$, and so hold the prospect of explaining a variety of experimental puzzles.

\section{Acknowledgments}

This research was supported by the National Science Foundation under grants DMR-0308945 (T.S.), DMR9985255 (L.B.), DMR-0098226 (S.S.) and DMR-0210790, PHY-9907949 (M.P.A.F.). We would also like to acknowledges funding from the NEC Corporation (T.S.), the Packard Foundation (L.B.), the Alfred P. Sloan Foundation (T.S., L.B.), a Pappalardo Fellowship (A.V.) and an award from The Research Corporation (T.S.). We thank the Aspen Center for Physics for hospitality.

\section{APPENDIX A: BERRY PHASES IN THE SJ MODEL}

The non-linear sigma model representation in Eq. (3.1) associates the Berry phases with a summation over the individual Berry phases of each spin. Each such contribution measures the area enclosed by the path of the spin on the unit sphere, and this is represented by $\mathcal{S}_{B}$ in Eq. (3.1). Upon transforming to the $z$ variables via Eq. (1.5), there 
is a simple way of measuring this area ${ }^{20.36 .38}$ : it is the Polyakov loop of the $\mathrm{U}(1)$ gauge field of the $\mathrm{CP}^{1}$ model. This connection suggests that the $\mathcal{S}_{B}$ in Eq. (3.13) should be replaced by

$$
\mathcal{S}_{B}^{\prime}=i \sum_{i} \epsilon_{i} a_{\tau}
$$

where $\epsilon_{i}$ is the cubic lattice, $\tau$-independent representation of the square lattice sublattice staggering factor $\epsilon_{r}$. One can now consider a 'modified' SJ model ${ }^{38}$ with action $\mathcal{S}_{\mathrm{S} J}^{\prime}=\mathcal{S}_{z}+\mathcal{S}_{a}+\mathcal{S}_{B}^{\prime}$ defined by Eqs. (3.11), (3.12) and (A1). This appendix will argue that the properties of $\mathcal{S}_{\mathrm{SJ}}$ and $\mathcal{S}_{\mathrm{SJ}}^{\prime}$ are very similar, and universal features are identical.

First, we show that for $t=0$ (this is deep in the VBS phase), the two theories are, in fact, exactly equivalent. In this limit, we can proceed with a duality mapping as in Section IVA and obtain a dual representation of $\mathcal{S}_{\mathrm{SJ}}$ which is Eq. (4.9) with $\mathbf{A}=0$ :

$$
\mathcal{S}=\frac{1}{2 K}(\boldsymbol{\Delta}(\chi+\vartheta))^{2}
$$

Proceeding with the analogous duality transformation to $\mathcal{S}_{\mathrm{SJ}}^{\prime}$, we find instead

$$
\mathcal{S}^{\prime}=\frac{1}{2 K}\left(\Delta \chi+\mathbf{B}_{0}\right)^{2}
$$

where $\mathbf{B}_{0}$ is a fixed integer-valued field on the links of the dual lattice chosen so that

$$
\boldsymbol{\Delta} \times \mathbf{B}_{0}=\epsilon \hat{\boldsymbol{\tau}}
$$

where $\hat{\boldsymbol{\tau}}$ is a unit vector in the $\tau$ direction. A convenient choice for $\mathbf{B}_{0}$ is shown in Fig 6 . Now note that we can write

$$
\mathbf{B}_{0}=\Delta \vartheta+\Delta \times \boldsymbol{\beta}
$$

with $\vartheta_{r}$ defined below Eq. (4.4) and shown in Fig $\boldsymbol{\beta}$ is a fixed vector field on the links of the dual lattice with only its temporal components non-zero as shown in Fig [6]. It is now evident that Eqs. A3 and A5 are exactly equivalent to Eq. (A2), as the couplings between $\boldsymbol{\beta}$ and $\chi, \vartheta$ vanish.

Moving to the general case with $t \neq 0$, let us examine the fate of the modified SJ model for $N=1$ as in Section IVA In this case, Eq. (4.9) is replaced by

$$
\mathcal{S}^{\prime}=\frac{1}{2 \tilde{t}}(\boldsymbol{\Delta} \times \mathbf{A})^{2}+\frac{1}{2 K}(\boldsymbol{\Delta}(\chi+\vartheta)+\mathbf{A}+\boldsymbol{\Delta} \times \boldsymbol{\beta})^{2} .
$$

It is now not difficult to show that the additional term associated with $\boldsymbol{\beta}$ above makes little difference to the universal properties of theory: integrating out the massive A modes is now a little more involved, but the final theory for $\chi$ has the same structure as that in Section IVA Further details may be found in Ref. 20.

Similar comments apply to the modified SJ model at $N=2$, with easy plane anisotropy, as discussed in Appendix B

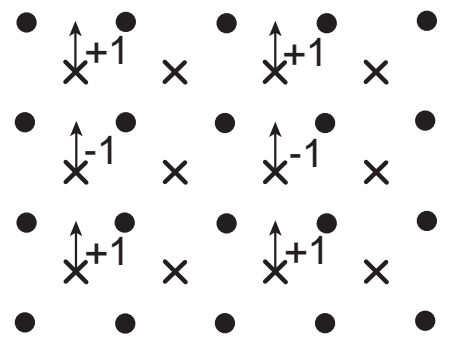

(a)

$$
\begin{aligned}
& +1 / 8-1 / 8+1 / 8-1 / 8 \\
& \times \times \times \\
& -1 / 8+1 / 8-1 / 8+1 / 8 \\
& \times \times \times \times \\
& +1 / 8-1 / 8+1 / 8 \quad-1 / 8
\end{aligned}
$$

(b)

FIG. 6: Specification of the non-zero values of the fixed fields (a) $\mathbf{B}_{0}$ and (b) $\boldsymbol{\beta}$. The circles are the sites of the direct lattice, $j$, while the crosses are the sites of the dual lattice, $\bar{j}$; the latter are also offset by half a lattice spacing in the direction out of the paper (the $\mu=\tau$ direction). The $B_{0 \mu}$ are all zero for $\mu=\tau, x$, while the only non-zero values of $B_{0 y}$ are shown in (a). Only the $\mu=\tau$ components of $\beta_{\mu}$ are non-zero, and these are shown in (b).

\section{APPENDIX B: DUALITY TRANSFORMATION WITH EASY PLANE ANISOTROPY}

The duality transformation for the SJ model at $N=2$ in the easy plane limit proceeds very similarly to that of the $N=1$ case discussed in Section IVA We begin by rewriting the 'boson hopping' term in Eq. (5.5) in a Villain approximation:

$$
\mathcal{S}_{z} \rightarrow \sum_{\ell, \alpha}\left[\frac{1}{2 \tilde{t}}\left|\mathbf{j}_{\alpha}\right|^{2}-i \mathbf{j}_{\alpha} \cdot\left(\boldsymbol{\Delta} \phi_{\alpha}-\mathbf{a}\right)\right],
$$

where $\alpha=1,2$ labels the two species of bosons and $\mathbf{j}_{\alpha}$ are the corresponding integer valued currents defined on the links of the square lattice. Proceeding as before in Section IVA integrating out the $\phi_{\alpha}$ fields leads to the current conservation conditions

$$
\Delta \cdot \mathbf{j}_{\alpha}=0 .
$$

which can be solved by writing

$$
\mathbf{j}_{\alpha}=\boldsymbol{\Delta} \times \mathbf{A}_{\alpha}
$$

with $\mathbf{A}_{\alpha}$ integer fields. We treat the gauge field kinetic energy term as in Section IVA by first decoupling it with the $\mathbf{b}$ field, and then summing over the integer $\mathbf{q}$ to obtain

$$
\mathbf{b}-\boldsymbol{\Delta} \vartheta=\mathbf{B}
$$


with $\mathbf{B}$ an integer field. Integrating over the gauge field a now replaces Eq. (4.7) by

$$
\boldsymbol{\Delta} \times \mathbf{B}=\mathbf{j}_{1}+\mathbf{j}_{2}
$$

This may be solved by writing

$$
\mathbf{B}=\mathbf{A}_{1}+\mathbf{A}_{2}+\boldsymbol{\Delta} \chi
$$

with $\chi$ an integer. The action then reads

$$
\begin{aligned}
\mathcal{S}=\sum[ & \frac{1}{2 \tilde{t}}\left(\boldsymbol{\Delta} \times \mathbf{A}_{1}\right)^{2}+\frac{1}{2 \tilde{t}}\left(\boldsymbol{\Delta} \times \mathbf{A}_{2}\right)^{2} \\
& \left.+\frac{1}{2 K}\left(\boldsymbol{\Delta}(\chi+\vartheta)+\mathbf{A}_{1}+\mathbf{A}_{2}\right)^{2}\right] .
\end{aligned}
$$

As in Eq. (4.10) we may soften the integer constraints on $\mathbf{A}_{\alpha}, \chi$ by adding the terms

$$
-t \cos \left(2 \pi \mathbf{A}_{1}\right)-t \cos \left(2 \pi \mathbf{A}_{2}\right)-\sum_{n} \lambda_{n} \cos (2 \pi n \chi)
$$

with $n$ running over all positive integers. Now we can shift $\chi \rightarrow \tilde{\chi}=\chi+\vartheta$. Then we can put $2 \pi \chi=\theta_{1}-\theta_{2}$, and integrate over both phase fields, $\theta_{\alpha}$, leaving the partition function unchanged up to an overall multiplicative constant. Upon shifting the two fields $\mathbf{A}_{1} \rightarrow \mathbf{A}_{1}-\boldsymbol{\Delta} \theta_{1} / 2 \pi$ and $\mathbf{A}_{2} \rightarrow \mathbf{A}_{2}+\boldsymbol{\Delta} \theta_{2} / 2 \pi$, the last term in Eq. (B7) takes the form $\left(\mathbf{A}_{1}+\mathbf{A}_{2}\right)^{2}$. We can then define $\mathbf{A}_{+}=\mathbf{A}_{1}+\mathbf{A}_{2}$ and $\mathbf{A}=\pi\left(\mathbf{A}_{1}-\mathbf{A}_{2}\right)$, and integrate out the massive field $\mathbf{A}_{+}$. Up to irrelevant terms we thereby obtain for the full action:

$$
\begin{gathered}
\mathcal{S}=\mathcal{S}_{\lambda}+\sum\left[\frac{4 \pi^{2}}{\tilde{t}}(\boldsymbol{\Delta} \times \mathbf{A})^{2}-t \cos \left(\boldsymbol{\Delta} \theta_{1}-\mathbf{A}\right)\right. \\
\left.-t \cos \left(\boldsymbol{\Delta} \theta_{2}-\mathbf{A}\right)\right] \\
\mathcal{S}_{\lambda}=-\sum\left[\sum_{n} \lambda_{n} \cos \left(n\left(\theta_{1}-\theta_{2}-2 \pi \vartheta\right)\right)\right]
\end{gathered}
$$

Once again as $e^{2 i \pi \vartheta}$ oscillates on four sublattices, for smooth variations of $\theta_{1,2}$, the lowest value of $n$ that survives is $n=4$. We may therefore replace $\mathcal{S}_{\lambda}$ by

$$
\mathcal{S}_{\lambda}=-\sum\left[\lambda \cos \left(4\left(\theta_{1}-\theta_{2}\right)\right)\right]
$$

with $\lambda \equiv \lambda_{4}$. The resulting action is then a 'hard-spin' version of the action $\mathcal{S}_{z}$ in Eq. (5.6) of Section $\nabla$ with the identification of the vortex operators, $\psi_{\alpha} \sim e^{i \theta_{\alpha}}$.

\section{APPENDIX C: ESTIMATE OF MONOPOLE SCALING DIMENSION}

We can ask about the answer for all these scaling dimensions that would be obtained by first integrating out the matter fields, truncating the resulting gauge action to quadratic order, and using that theory to calculate the scaling dimension. This would be an estimate, albeit uncontrolled; one might hope that it will correctly capture the trends.

Anyway, it turns out that this can be done without serious calculation. First, note that in this procedure the answer depends only on the universal conductivity of the matter fields at the transition ignoring all coupling to the gauge field. The higher this universal conductivity the higher the instanton anomalous dimension.

The simplest case is the $N=1$ SJ model. Here the relevant universal conductivity is that of a single boson species. We know that at this transition the four instanton operator is irrelevant. Now in the $N=2$ cases with either easy plane or full $\mathrm{SU}(2)$ symmetry, it is clear that the universal conductivity will only be larger than at $N=1$. Thus we should expect a higher anamolous dimension. This would then predict irrelevance of the four instanton term with or without easy plane anisotropy for the physical case of $N=2$ in agreement with other expectations.

\section{APPENDIX D: SJ MODELS IN ONE DIMENSION}

There is a close and useful analogy between some of the phenomena explored in this paper and corresponding ones in one spatial dimension. Specifically consider a one dimensional spin-1/2 magnet in the presence of some easy plane anisotropy. The analog of the Néel phase in $d=1$ is a phase with power law correlators for the staggered XY magnetization. This phase may be described as a Luttinger liquid. There is a direct second order transition between this phase and a VBS phase where there is spontaneous dimerization of the spin chain.

A useful theoretical description of this transition is obtained by focusing on vortices in the space-time configuration of the staggered XY order parameter field. From a quantum mechanical point of view such vortices correspond to phase-slip (or instanton) events. It is wellknown that a $2 \pi$ phase slip event carries a momentum $\pi$, and hence is not allowed as a term in the Hamiltonian. (In an equivalent description of this Luttinger liquid phase in terms of interacting spinless fermions, these $2 \pi$ phase slips correspond to interchange of left and right movers). In the VBS phase these phase slip events have proliferated. Indeed it is precisely the $\pi$ momentum that is carried by the $2 \pi$ phase slip that is responsible for the broken translation symmetry of the VBS. A convenient order parameter for the VBS phase is therefore provided by the $2 \pi$ phase slip operator.

Though $2 \pi$ phase slip terms are not allowed in the Hamiltonian $4 \pi$ phase slips (which carry zero crystal momentum) are clearly allowed. The transition from the Luttinger liquid to the VBS is driven by the proliferation of these $4 \pi$ phase slips. These 'doubled' phase slips are irrelevant throughout the Luttinger liquid phase and are marginally irrelevant at the critical fixed point. 
The analogy with the two dimensional situations considered in this paper is now clear. The $2 \pi$ phase slip is the direct analog of the skyrmion tunneling (i.e single instanton) event. In both $d=1,2$ the VBS phase is understood as a condensate of the appropriate single instanton event. In $d=1$ the transition is driven by doubled instanton events (similar to the quadrupling of instantons in two dimensions) which stay irrelevant at the critical fixed point. At a formal level it is possible to construct an appropriate 'SJ' model that correctly describes the transition even in $d=1$.

A complete presentation closely related to the discussion in this appendix appears in Ref. 38 .

\section{APPENDIX E: DIRECT DERIVATION OF DUAL MERON ACTION}

We pass from Eq. (9.1) to the analog of a $\mathrm{CP}^{1}$ representation by letting

$$
\begin{aligned}
e^{i \phi_{r}} & =b_{1 r}^{\dagger} b_{2 r}=e^{-i\left(\phi_{1 r}-\phi_{2 r}\right)} \\
n_{r} & =\frac{n_{1 r}-n_{2 r}+1}{2} \\
n_{1 r}+n_{2 r} & =\epsilon_{r} .
\end{aligned}
$$

Here $b_{1,2}=e^{i \phi_{1,2}}$ represent charge- $\pm 1 / 2$ bosonic operators and $n_{1,2}$ are the corresponding boson numbers, and $\epsilon_{r}$ was defined in Eq. (1.3). Note that $b_{1,2}$ are not canonical Bose operators, and the relevant commutation relations here are $\left[n_{1}, \phi_{1}\right]=-i$ and $\left[n_{2}, \phi_{2}\right]=-i$. The eigenvalues of $n_{1,2}$ are integers which run from $-\infty$ to $\infty$. As is usual, there is a gauge redundancy associated with an arbitrary choice of the local phase of the $b_{1,2}$ fields. The last equation is a constraint that requires the total number of both species of bosons to be fixed at +1 on the A sublattice and -1 on the $B$ sublattice. The left-hand side of this constraint equation is precisely the generator of the local gauge transformation. We have chosen to stagger this gauge charge on the two sublattices.

The Hamiltonian in Eq. 9.1 is readily rewritten in terms of these new variables:

$$
\begin{aligned}
H & =H_{U}+H_{t} \\
H_{U} & =\frac{U}{2} \sum_{r}\left[\left(n_{1 r}-\epsilon_{r} n_{0}\right)^{2}+\left(n_{2 r}-\epsilon_{r} n_{0}\right)^{2}\right] \\
H_{t} & =-t \sum_{\left\langle r r^{\prime}\right\rangle}\left[\left(b_{1 r}^{\dagger} b_{2 r}\right)\left(b_{2 r^{\prime}}^{\dagger} b_{1 r^{\prime}}\right)+\text { H.c. }\right]
\end{aligned}
$$

We have introduced a term proportional to $n_{0}$ which describes a 'chemical potential' for the total on-site gauge charge. As the total gauge charge is fixed to \pm 1 on each site, this addition is completely innocuous (for any value of $\left.n_{0}\right)$. Later we will choose $n_{0}$ appropriately to ensure that the $n_{1,2}$ fields have zero mean value. While this step is not necessary, it is convenient, and will be commented upon further at the appropriate point.
Now we proceed to a path integral representation to write

$$
\begin{aligned}
\mathcal{S} & =\mathcal{S}_{U}+\mathcal{S}_{\tau}+\mathcal{S}_{a_{0}}+\mathcal{S}_{t} \\
\mathcal{S}_{U} & =\sum_{r} \int d \tau \frac{U}{2} \sum_{r}\left[\left(n_{1 r}-\epsilon_{r} n_{0}\right)^{2}+\left(n_{2 r}-\epsilon_{r} n_{o}\right)^{2}\right] \\
\mathcal{S}_{\tau} & =\sum_{r} \int d \tau\left[i n_{1 r} \partial_{\tau} \phi_{1 r}+i n_{2 r} \partial_{\tau} \phi_{2 r}\right] \\
\mathcal{S}_{a_{0}} & =\sum_{r} \int d \tau\left[i a_{0}\left(n_{1 r}+n_{2 r}-\epsilon_{r}\right)\right] \\
\mathcal{S}_{t} & =\int d \tau H_{t}
\end{aligned}
$$

As usual, it is assumed that there is a fine discretization of the imaginary time index $\tau$, and that the integervalued boson numbers $n_{1 r}, n_{2 r}$ live on the temporal links at each spatial point. The 'gauge' constraint is imposed by means of a Lagrange multiplier field $a_{0}$ which will be interpreted as the time-component of a gauge field. We now proceed as is usual in slave particle theories of correlated systems. We decouple the interactions in $H_{t}$ using a complex auxiliary field $\chi_{r r^{\prime}}$ defined on each spatial link to write

$$
\begin{aligned}
e^{-\mathcal{S}_{t}}= & \prod_{\left\langle r r^{\prime}\right\rangle} \int d \chi_{r r^{\prime}} \exp \left[-\frac{\left|\chi_{r r^{\prime}}\right|^{2}}{t}\right. \\
& \left.+\chi_{r r^{\prime}} \sum_{\alpha=1}^{2} e^{i\left(\phi_{\alpha r}-\phi_{\alpha r^{\prime}}\right)}+\text { c.c. }\right],
\end{aligned}
$$

with $\chi_{r r^{\prime}}=\chi_{r^{\prime} r}^{*}$. The fluctuations in the amplitude of the $\chi$ field are expected to be innocuous. Hence we will write

$$
\chi_{r r^{\prime}} \approx \chi_{0} e^{i a_{r r^{\prime}}}
$$

with $\chi_{0}$ a constant that simply renormalizes the boson hopping amplitude. As usual the $a_{r r^{\prime}}=-a_{r^{\prime} r}$ will be interpreted as the spatial component of a gauge field. The full action now is invariant under the gauge transformation

$$
\begin{aligned}
e^{i \phi_{\alpha r}(\tau)} & \rightarrow e^{i \gamma_{r}(\tau)} e^{i \phi_{\alpha r}(\tau)} \\
a_{0} & \rightarrow a_{0}-\frac{d \gamma_{r}(\tau)}{d \tau} \\
a_{r r^{\prime}} & \rightarrow a_{r r^{\prime}}-\gamma_{r}(\tau)+\gamma_{r^{\prime}}(\tau)
\end{aligned}
$$

where $\alpha=1,2$. To examine universal critical properties near the phase transitions of interest it is legitimate to add various possible local terms that are consistent with the global symmetries and gauge structure of the action. It is particularly useful to add a 'kinetic energy' term for the gauge fields on all plaquettes (spatial and space-time) in Villain form:

$$
\begin{aligned}
\mathcal{L}_{E} & =\frac{u}{2} E_{i}^{2}+i E_{i}\left(\partial_{\tau} a_{i}-\Delta_{i} a_{0}\right) \\
\mathcal{L}_{B} & =\frac{u}{2} B^{2}+i B\left(\epsilon_{i j} \Delta_{i} a_{j}\right) .
\end{aligned}
$$


Here $E_{i}$ is an integer-valued 'electric field' defined on the spatial links at each time-slice $(i, j$ extend over the spatial co-ordinates $x, y)$ and $B$ is the corresponding integer-valued magnetic field on a spatial plaquette. We have introduced the vector notation $a_{i}=\left(a_{x}, a_{y}\right)=$ $\left(a_{\vec{r}, \vec{r}+\hat{x}}, a_{\vec{r}, \vec{r}+\hat{y}}\right)$. For simplicity we have chosen the same constant $u$ multiplying the $E_{i}^{2}$ and $B^{2}$ terms. The original microscopic action is formally obtained in the large- $u$ limit $(u \rightarrow \infty)$.

To proceed it is first useful to note that the background gauge charge present in this formulation will lead to a background electric field about which the true electric field will actually fluctuate. It will be convenient to incorporate this effect by finding a suitable mean field for the various fields in the action. Consider a mean field (saddle point) of the action where the non-zero expectation values are

$$
\begin{aligned}
\left\langle n_{1}\right\rangle=\left\langle n_{2}\right\rangle & =\bar{n} \\
\left\langle a_{0}\right\rangle & =\bar{a}_{0} \\
\left\langle E_{i}\right\rangle & =E_{i 0} .
\end{aligned}
$$

The saddle point equations are obtained by varying the action with respect to these fields:

$$
\begin{aligned}
u E_{i 0} & =i \Delta_{i} \bar{a}_{0} \\
\Delta_{i} E_{i 0} & =-2 \bar{n}+\epsilon_{r} \\
U\left(\bar{n}-\epsilon_{r} n_{0}\right) & =-i \bar{a}_{0}
\end{aligned}
$$

We now use our freedom in choosing the constant $n_{0}$ to set

$$
n_{0}=\frac{i \bar{a}_{0} \epsilon_{r}}{U}
$$

so that $\bar{n}=0$. One may worry that this special choice of $n_{0}$ might indicate some non-generic nature of the resulting theory. We note, however, that qualitatively identical results are obtained for any other choice of $n_{0}$ - in the dual action with such a choice the merons see some nonzero but spatially oscillating flux. Having a zero spatial average, this flux does not qualitatively effect the low energy (extended) meron states. The above choice simply renders the low energy behavior more transparent. We then have

$$
\Delta_{i} E_{i 0}=\epsilon_{r}
$$

Note also that $E_{i 0}$ is the gradient of a potential determined by $a_{0}$. These conditions determine $E_{i 0}$ (the background electric field) uniquely to have the value $1 / 4$ oriented from the $A$ to $B$ sublattice.

We may now examine the full theory by first shifting $a_{0}=\bar{a}_{0}+\delta a_{0}$. Straightforward manipulation shows that the electric field now fluctuates about a background value $E_{i 0}$ so that the $E_{i}$ dependent terms in the action read

$$
\mathcal{L}_{E}=\frac{u}{2}\left(E_{i}-E_{i 0}\right)^{2}+i E_{i}\left(\partial_{\tau} a_{i}-\Delta_{i} a_{0}\right)
$$

All other terms remain unchanged (after the replacing $a_{0}$ with $\delta a_{0}$ ). We may now dualize this action as in previous sections to directly derive the dual meron action of Section $\nabla$

\section{APPENDIX F: BREAKDOWN OF THE 'SCREENING ARGUMENT' IN THE MONOPOLE GAS}

In this Appendix we will consider a simple toy model of a compact $\mathrm{U}(1)$ gauge theory without Berry phases which can be shown to possess a deconfined critical point. This will enable us to understand clearly the claim of Section IVB that the specific monopole gases that obtain at the critical points studied in this paper evade the general monopole screening arguments ${ }^{29.30}$ for a three dimensional Coulomb gas with logarithmic interactions.

We consider a model of charge $n$ bosons $(n \geq 4)$ coupled to a compact $\mathrm{U}(1)$ gauge field in $D=2+1$ dimensions with Euclidean action

$$
\mathcal{S}=-J \sum \cos (\boldsymbol{\Delta} \phi-n \mathbf{a})-K \sum_{P} \cos (\boldsymbol{\Delta} \times \mathbf{a}) .
$$

Here $e^{i \phi_{i}}$ represents a boson field on the sites $i$ of a three dimensional cubic lattice. The sum in the first term is over the links of the lattice, while that in the second term is over the plaquettes $P$. The field $a_{i j} \equiv a_{i j}+2 \pi$ is a compact $\mathrm{U}(1)$ gauge field, and the integer $n$ is the charge of the boson. This model has two phases. For large $J$, there is a Higgs phase where the boson field has 'condensed'. Following the arguments of Ref. 67, the effective theory of this phase is a $Z_{n}$ gauge theory in its deconfined phase in $2+1$ dimensions. The excitations in this phase are stable vortices that carry flux $2 \pi q / n$, for $q=1, \ldots \ldots . n-1$, of the gauge field $a$. For small $J$, on the other hand, there is a different phase which is associated with confinement of the $\mathrm{U}(1)$ gauge theory. In particular, the $Z_{n}$ vortices that appear in the Higgs phase are no longer present in the spectrum. As also argued in Ref. 67, the transition between these two phases is actually described by that in a $Z_{n}$ gauge theory. This latter theory is dual to the global $Z_{n}$ clock model - for $n \geq$ 4 the clock anisotropy is irrelevant and the universality class is $3 D \mathrm{XY}$.

The $n=1$ case of Eq. (F1) was considered in Ref. 28 . For this case, the 'clock' anisotropy is strongly relevant (it rounds out the transition into a crossover), and the physics is very different from $n \geq 4$.

Formally, the action above is readily dualized (as in many of the other examples discussed at length in previous sections). The dual action takes the form

$$
\mathcal{S}_{\text {dual }}=-t \sum[\cos (2 \pi \Delta \chi)-\lambda \cos (2 \pi n \chi)]
$$

and has the expected structure of a global XY model with $n$-fold anisotropy.

It is useful, for our purposes, to have a physical interpretation of these results. In the Higgs phase, the vorticity of the $\phi$ field is quantized in units of $2 \pi / n$ (as is natural for a charge $n$ condensate). The presence of instantons implies that the total flux can change by $2 \pi$, so that $n$ of these vortices can appear or disappear together. Thus the vortices only carry a $Z_{n}$ quantum number. The 
dual description focuses on these $Z_{n}$ vortices. Without instantons, the $2 \pi / n$ vortex is globally conserved and its physics is described by a global XY model (this is just the duality in Ref. 55). The presence of instantons leads to the $n$-fold anisotropy (the $\lambda$ term in Eq. (F2) for this global XY model, leading to the global $Z_{n}$ model. Thus the irrelevance of the $n$-fold anisotropy, for $n \geq 4$, should be interpreted as the irrelevance of instantons at the transition. Indeed the XY universality class, is the exact dual of the condensation transition of the charge $n$ boson coupled to a non-compact $\mathrm{U}(1)$ gauge field. ${ }^{55}$

Now let us analyze the transition in the 'RPA' approach outlined in Section IVB The transition is associated with the condensation of the $e^{i \phi}$ field. We therefore integrate out this field in the presence of a non-trivial gauge potential, and truncate the resulting gauge action to quadratic order (initially ignoring instantons). The result is, as in Eq. (4.12),

$$
\mathcal{S}_{G}=\int \frac{d^{3} K}{(2 \pi)^{3}} \sigma_{0} n^{2}|K|\left|\mathbf{a}_{T}(\mathbf{K})\right|^{2}+\ldots
$$

Here $\sigma_{0}$ is a universal constant, and $\mathbf{a}_{T}$ is the transverse component of the gauge field. The Maxwell term present in the bare action is less important at long distances than the term in the action displayed above, and we have dropped it. We now examine the stability of $\mathcal{S}_{G}$ to instantons. First, we dualize $\mathcal{S}_{G}$ to obtain 28,29

$$
\mathcal{S}_{G, \text { dual }}=\int \frac{d^{3} K}{(2 \pi)^{3}} \frac{K^{3}}{\sigma_{0}}|\chi(K)|^{2}-\sum \lambda \cos (2 \pi n \chi) .
$$

The last term represents instanton events. Note the $K^{3}$ in the first term. Now the $\lambda$ term will in general generate a $K^{2}$ term in the Gaussian $\chi$ action which will then eventually make instantons relevant. A fine-tuning is required ${ }^{29.30}$ to prevent the generation of the $K^{2}$ term in this argument, and for the present model we can now easily see that this fine tuning is automatic at the critical point of the gauge theory in Eq. (F1).

The key is to note that the logarithmic interaction between the monopoles is equivalent to the statement that the correlators of $e^{2 i \pi n \chi}$ decay as a power law (at the fixed point without monopoles). In the theory of the monopoles in Eq. (F2), the $e^{2 i \pi \chi}$ field is at the critical point of the 3D $X Y$ model. The RPA theory approximates this non-trivial interacting critical theory by an equivalent Gaussian theory, which also happens to give power law correlations for the $e^{2 i \pi \chi}$ field (this is a property of the $K^{3}|\chi|^{2}$ form). In the full theory in Eq. (F2), the screening of monopole interactions is associated with corrections higher order in $\lambda$. In the context of conformal perturbation theory about the critical point of Eq. (F2), however, it is clear that these higher order effects in $\lambda$ actually represent shifts in the position of the critical point, and not any changes in the scaling dimensions of operators.

Hence for the Néel-VBS transition we conclude that the 'nä̈ve' computation of monopole scaling dimensions in the large $N$ limit $^{35}$ is actually correct, and that we should neglect screening between multiple monopoles in determining this scaling dimension. The latter effects are more correctly accounted for by shifting the position of the critical point.

Note that for the $n=1$ case of Eq. (F1) considered in Ref. 28, computation of the scaling dimension of the monopole operator using Eq. (F2) shows that monopoles are relevant at the critical point. Indeed, they round out the transition to a crossover, and the monopoles are always in a screened plasma phase. So the conclusions of Refs. 2930 for this case are correct, but not for completely sound reasons.
1 L. D. Landau, E. M. Lifshitz, and E. M. Pitaevskii, Statistical Physics (Butterworth-Heinemann, New York, 1999).

2 K. G. Wilson and J. Kogut, Phys. Rep. 12, 75 (1974).

3 S. Sachdev, Quantum Phase Transitions, (Cambridge University Press, Cambridge, England, 1999).

${ }^{4}$ F. F. Assaad, M. Imada, and D. J. Scalapino, Phys. Rev. Lett. 77, 4592 (1996); Phys. Rev. B 56, 15001 (1997).

5 A. W. Sandvik, S. Daul, R. R. P. Singh, and D. J. Scalapino, Phys. Rev. Lett. 89, 247201 (2002).

6 P. Coleman, C. Pépin, Q. Si, and R. Ramazashvili, J. Phys: Condens. Matt. 13, 723 (2001).

7 G. R. Stewart, Rev. Mod. Phys. 73, 797 (2001).

8 R. B. Laughlin, Adv. Phys. 47, 943 (1998).

9 T. Senthil, A. Vishwanath, L. Balents, S. Sachdev, and M. P. A. Fisher, cond-mat/0311326

${ }^{10}$ F. D. M. Haldane, Phys. Rev. B 25, 4925 (1982).

11 N. Read and S. Sachdev, Phys. Rev. Lett. 62, 1694 (1989).

12 K. P. Schmidt and G. S. Uhrig, Phys. Rev. Lett. 90, 227204 (2003).
13 P. W. Anderson, Science 235, 1196 (1987).

14 S. A. Kivelson, D. S. Rokhsar, and J. P. Sethna, Phys. Rev. B 35, 8865 (1987); E. Fradkin and S. A. Kivelson, Mod. Phys. Lett. B 4, 225 (1990).

15 N. Read and S. Sachdev, Phys. Rev. Lett. 66, 1773 (1991).

16 X. G. Wen, Phys. Rev. B 44, 2664 (1991).

17 T. Senthil and M. P. A. Fisher, Phys. Rev. B 62, 7850 (2000).

18 N. Read and S. Sachdev, Phys. Rev. B 42, 4568 (1990).

19 F. D. M. Haldane, Phys. Rev. Lett. 61, 1029 (1988).

20 S. Sachdev, to appear on cond-mat in Jan 2004.

${ }^{21}$ O. Motrunich and A. Vishwanath, cond-mat/0311222

22 A. D'Adda, P. Di Vecchia, and M. Lüscher, Nucl. Phys. B 146, 63 (1978); E. Witten, Nucl. Phys. B 149, 285 (1979).

23 E. Fradkin, D. A. Huse, R. Moessner, V. Oganesyan, and S. L. Sondhi, cond-mat/0311353 E. Ardonne, P. Fendley, and E. Fradkin, cond-mat/0311466.

24 A. Vishwanath, L. Balents, and T. Senthil, cond-mat/0311085 
25 A. M. Polyakov, Gauge Fields and Strings, Harwood Academic, New York (1987).

${ }^{26}$ L. B. Ioffe and A. I. Larkin, Phys. Rev. B 39, 8988 (1989).

27 X.-G. Wen, Phys. Rev. B 65, 165113 (2002)

${ }^{28}$ H. Kleinert, F. S. Nogueira, and A. Sudbø, Phys. Rev. Lett. 88, 232001 (2002).

29 I. F. Herbut and B. H. Seradjeh, Phys. Rev. Lett. 91 171601 (2003).

${ }^{30}$ I. F. Herbut, B. H. Seradjeh, S. Sachdev, and G. Murthy, Phys. Rev. B 68, 195110 (2003).

31 It seems likely that the non-relativistic, spinon Fermi surface state discussed in Ref. 30 is unlikely to behave like a critical system, and so will not suppress instantons without fine tuning.

32 S. Chakravarty, B. I. Halperin, and D. R. Nelson, Phys. Rev. B 39, 2344 (1989).

33 A. V. Chubukov, S. Sachdev, and J. Ye, Phys. Rev. B 49, 11919 (1994)

34 X. G. Wen and A. Zee, Phys. Rev. Lett. 61, 1025 (1988); E. Fradkin and M. Stone, Phys. Rev. B 38, 7215 (1988); T. Dombre and N. Read, Phys. Rev. B 38, 7181 (1988).

35 G. Murthy and S. Sachdev, Nucl. Phys. B 344557 (1990).

36 S. Sachdev and R. Jalabert, Mod. Phys. Lett. B 4, 1043 (1990).

37 At large- $N$ even the single monopole operator is irrelevant so that even without Berry phases a 'deconfined' critical fixed point obtains. However this is special to large- $N$ and does not hold at $N=1$ (or in the easy plane limit at $N=2$ ).

38 S. Sachdev and K. Park, Annals of Physics, N.Y. 298, 58 (2002).

39 M. Kamal and G. Murthy, Phys. Rev. Lett. 71, 1911 (1993).

40 G. Misguich and C. Lhuillier in Frustrated spin systems, H. T. Diep ed., World-Scientific, Singapore (2003), cond-mat/0310405

41 M. P. Gelfand, R. R. P. Singh, and D. A. Huse, Phys. Rev. B 40, 10801 (1989).

42 E. Dagotto and A. Moreo, Phys. Rev. Lett. 63, 2148 (1989); R. R. P. Singh and R. Narayanan, Phys. Rev. Lett. 65, 1072 (1990); H. J. Schulz and T. A. L. Ziman, Europhys. Lett. 18, 355 (1992); H. J. Schulz, T. A. L. Ziman, and D. Poilblanc, J. Phys. I (France) 6, 675 (1996).

43 V. N. Kotov, J. Oitmaa, O. P. Sushkov, and Z. Weihong, Phys. Rev. B 60, 14613 (1999); R. R. P. Singh, Z. Weihong, C. J. Hamer, and J. Oitmaa, Phys. Rev. B 60, 7278 (1999); V. N. Kotov and O. P. Sushkov, Phys. Rev. B 61, 11820 (2000); O. P. Sushkov, J. Oitmaa, and Z. Weihong, Phys. Rev. B 66, 054401 (2002).

44 M. S. L. du Croo de Jongh, J. M. J. van Leeuwen, and W. van Saarloos, Phys. Rev. B 62, 14844 (2000).

45 K. Harada, N. Kawashima, and M. Troyer, Phys. Rev. Lett. 90, 117203 (2003).
46 M. Troyer, M. Imada, and K. Ueda, J. Phys. Soc. Jpn 66 2957 (1997).

47 M. Matsumoto, C. Yasuda, S. Todo, and H. Takayama, Phys. Rev. B 65, 014407 (2002).

48 C. Lannert, M. P. A. Fisher, and T. Senthil, Phys. Rev. B 63, 134510 (2001).

49 L. Capriotti, F. Becca, A. Parola, and S. Sorella, Phys. Rev. Lett. 87, 097201 (2001).

50 J. M. Carmona, A. Pelissetto, and E. Vicari, Phys. Rev. B 61, 15136 (2000).

51 R. Jalabert and S. Sachdev, Phys. Rev. B 44, 686 (1991); S. Sachdev and M. Vojta, J. Phys. Soc. Jpn. 69, Suppl. B, 1 (2000).

${ }^{52}$ V. Yu. Irkhin, A. A. Katanin, and M. I. Katsnelson, Phys. Rev. B 54, 11953 (1996).

53 A. V. Chubukov and O. A. Starykh, Phys. Rev. B 52, 440 (1995).

${ }^{54}$ G. Murthy, Phys. Rev. Lett. 67, 911 (1991).

55 C. Dasgupta and B. I. Halperin, Phys. Rev. Lett. 47, 1556 (1981).

56 L. Balents and E. Frey, Phys. Rev. B 55, 1050 (1997).

57 T. Senthil and Matthew P.A. Fisher, Phys. Rev. Lett. 86, 292-295 (2001); Phys. Rev. B 63, 134521 (2001).

58 S. Sachdev and N. Read, Int. J. Mod. Phys. B 5, 219 (1991). The Higgs fields $Q_{x}$ and $Q_{y}$ in Section VIIIA are the Higgs fields $Q_{3, x}$ and $Q_{3, y}$ of the $(q, q)$ SRO phase of this paper.

59 S. Sachdev, Phys. Rev. B 45, 12377 (1992).

60 R. Moessner and S. L. Sondhi, Phys. Rev. Lett. 86, 1881 (2001); R. Moessner, S. L. Sondhi, and E. Fradkin, Phys. Rev. B 65, 024504 (2002).

61 C.-H. Chung, J. B. Marston, and R. H. McKenzie, J. Phys.: Condens. Matter, 135159 (2001).

62 C.-H. Chung, J. B Marston, and S. Sachdev, Phys. Rev. B 64, 134407 (2001).

63 C.-H. Chung, K. Voelker, and Y.-B. Kim, Phys. Rev. B 68, 094412 (2003).

64 J.-S. Bernier, C.-H. Chung, Y.-B. Kim, and S. Sachdev, cond-mat/0310504

65 As shown in Ref. 58, in the bosonic mean-field theory, the Higgs field $Q_{i}$ has the same symmetry transformation properties as the spinon pair field $\epsilon_{\alpha \beta} z_{\alpha} \partial_{i} z_{\beta}$. Note that the requirement of $S U(2)$ invariance leads to a spatial vector index on $Q_{i}$, and makes it odd under certain reflections. In the easy plane case, we can form a single charge 2 Higgs field as $z_{\alpha}^{2}$, and so the single Higgs field model in Eq. 8.1] can apply. In the $S U(2)$ invariant models on the square lattice of Ref. 58, the two-Higgs field model in Eq. (8.3) is necessary.

66 T. Imai, C. P. Slichter, K. Yoshimura, and K. Kosuge Phys. Rev. Lett. 70, 1002 (1993).

67 E. Fradkin and S. Shenker, Phys. Rev. D 19, 3682 (1979). 